University of Tennessee Health Science Center

UTHSC Digital Commons

$12-2010$

\title{
Therapeutic Gene Delivery to Human Pancreatic Islets for Treatment of Diabetes and the Effect of TFO on Liver Fibrosis Induced by Bile Duct Ligation
}

Ravikiran Panakanti

University of Tennessee Health Science Center

Follow this and additional works at: https://dc.uthsc.edu/dissertations

Part of the Pharmacy and Pharmaceutical Sciences Commons

\section{Recommended Citation}

Panakanti, Ravikiran, "Therapeutic Gene Delivery to Human Pancreatic Islets for Treatment of Diabetes and the Effect of TFO on Liver Fibrosis Induced by Bile Duct Ligation" (2010). Theses and Dissertations (ETD). Paper 206. http://dx.doi.org/10.21007/etd.cghs.2010.0236. 


\title{
Therapeutic Gene Delivery to Human Pancreatic Islets for Treatment of Diabetes and the Effect of TFO on Liver Fibrosis Induced by Bile Duct Ligation
}

\begin{abstract}
Ex vivo gene transfer can improve the outcome of islet transplantation for treating Type I diabetes. Earlier we have shown co-expression of human vascular endothelial growth factor (hVEGF) and human interleukin-1 receptor antagonist (hl-1Ra) after transfection of plasmid DNA encoding these two genes. Due to poor transfection efficiency of plasmid DNA and the better known islet transduction efficiency of adenoviral (Adv) vectors, in this study, we constructed Adv-hVEGF-hIL-1Ra by cloning hVEGF and hIL-1Ra coding sequences and polyA signal under separate cytomegalovirus (CMV) promoters in Adenoquick plasmid (Ad 13.1). There was dose and time dependent expression of these genes after transduction of Adv-hVEGF-hIL-1Ra into human islets. The mRNA expression of hVEGF and hIL-1Ra was more than 100 times higher than that of the non-transduced and bicistronic plasmid transfected control islets.

Transduced islets were viable as evidenced by insulin release upon glucose challenge. Co-expression of hVEGF and hIL-1Ra by islets showed decrease in caspase-3 activity and apoptosis induced by a cocktail of inflammatory cytokines such as TNF- $\alpha$ IL-1ßand IFN- $\gamma$. Compared to non-treated or Adv-LacZ transduced islets, transduction of islets with Adv-hVEGF-hIL-1Ra prior to transplantation under the kidney capsules of diabetic NOD-SCID mice reduced the blood glucose levels, and increased serum insulin and c-peptide levels. Immunohistochemical staining of the islet bearing kidney sections at day 30 after transplantation was positive for human insulin, hVEGF and von Willebrand factor.

Further, we decided to replace VEGF with hepatocyte growth factor (HGF) as it not only can promote revascularization of islets but also increases $\beta$-cell proliferation. It can also protect the islets from apoptosis. Adv-hHGF-hIL-1Ra showed dose and time dependent expression of these genes after transduction into human islets and further showed significant decrease in caspase- 3 induced by the cytokines. Compared to un-transduced islets, transduction of islets with Adv-hHGF-hIL-1Ra at $1000 \mathrm{MOI}$ prior to transplantation under the kidney capsules of streptozotocin-induced-diabetic NOD-SCID mice reduced blood glucose levels, and increased serum insulin and c-peptide levels.
\end{abstract}

These results indicate that the bicistronic Adv vector efficiently expresses both growth factor and antiapoptotic genes, decreases apoptosis and improves the outcome of islet transplantation.

Liver fibrosis is a consequence of chronic liver disorders which lead to accumulation of extra cellular matrix (ECM). Particularly, there is an increased accumulation of collagen in the fibrotic liver. We have therefore used a triplex forming oligonucleotide (TFO) against the type $\alpha 1$ (I) collagen and evaluated, whether it can attenuate liver fibrosis induced by common bile duct ligation (CBDL). There was a significant decrease in hydroxyproline levels in TFO-treated groups compared to non-treated CBDL group. Masson's trichrome staining showed weak staining for collagen in TFO-treated liver tissues and there was a predominantly high staining in non-treated CBDL group. There was over expression of $\alpha$-smooth muscle actin ( $\alpha$-SMA), marker for myofibroblasts following CBDL. Western blotting analysis also revealed an increase in TGF- $\beta 1$ expression, which is a potential marker for fibrosis in the CBDL group compared to TFO-treated group. These results suggest that TFO can be used to downregulate Type I collagen gene expression and can alleviate liver fibrosis induced by common bile duct ligation.

\section{Document Type}

Dissertation

Degree Name

Doctor of Philosophy (PhD) 


\section{Program}

Pharmaceutical Sciences

Research Advisor

Ram I. Mahato, Ph.D.

\section{Keywords}

Adenoviral Vectors, Diabetes, Gene/Drug Delivery, Islet Transplantation, Liver Fibrosis, Triplex Forming Oligonucleotide

\section{Subject Categories}

Medicine and Health Sciences | Pharmacy and Pharmaceutical Sciences 


\title{
THERAPEUTIC GENE DELIVERY TO HUMAN PANCREATIC ISLETS FOR TREATMENT OF DIABETES AND THE EFFECT OF TFO ON LIVER FIBROSIS INDUCED BY BILE DUCT LIGATION
}

\author{
A Dissertation \\ Presented for \\ The Graduate Studies Council \\ The University of Tennessee \\ Health Science Center
}

\author{
In Partial Fulfillment \\ Of the Requirements for the Degree \\ Doctor of Philosophy \\ From The University of Tennessee
}

By

Ravikiran Panakanti

December 2010 
Portions of Chapter 1 (C) 2010 by CRC Press.

Portions of Chapter 2 (C) 2008 by American Chemical Society.

Portions of Chapter 3 (C) 2008 by Springer.

All other material $\odot 2010$ by Ravikiran Panakanti.

All rights reserved. 


\section{DEDICATION}

This dissertation is dedicated to my parents, Mr. Ramkishan Rao Panakanti and Mrs. Varalaxmi Panakanti and my brothers Rajeev and Raakesh for their endless love and continuous support. 


\section{ACKNOWLEDGEMENTS}

I could never have completed this daunting task without the help, support, guidance and efforts of a lot of people. First and foremost, I would like to express my deepest gratitude and sincere appreciation to my mentor Dr. Ram I. Mahato for providing me with an opportunity to work in his lab and instilling in me the qualities of being a good scientist. His encouragement and patience early on in my $\mathrm{PhD}$ were crucial for my graduate career. I am very grateful for his co-operation and continued support during my graduate career at the University of Tennessee Health Science Center. I would also like to thank my committee members, Dr. Ramareddy V. Guntaka, Dr. Ivan C. Gerling, Dr. John $\mathrm{K}$. Buolamwini and Dr. Yi Lu, for their invaluable suggestions, guidance and assistance over the years.

I would also like to thank Drs Ajit Narang, Kun Cheng, Zhaoyang Ye, Guofeng Cheng and Akshay Pratap for their valuable guidance and help early on in my PhD program. A special thank you to my friends and colleagues, Dr. Xiangxu Jia, Lin Zhu, Feng Li, Ningning Yang, Michael Danquah, Hao Wu and Dr. Wenli Lu for their support and wonderful company over all these years. I also extend my gratitude to my friends for their friendship and for making my stay in Memphis a memorable experience.

I would like to thank the University of Tennessee Health Science Center for providing me with the opportunity to pursue graduate education. I would like to thank my parents and my brothers. I would also like to thank all my family members for their love and support. Finally, I would like to thank Almighty for everything that I have today. 


\begin{abstract}
Ex vivo gene transfer can improve the outcome of islet transplantation for treating Type I diabetes. Earlier we have shown co-expression of human vascular endothelial growth factor (hVEGF) and human interleukin-1 receptor antagonist (hIL-1Ra) after transfection of plasmid DNA encoding these two genes. Due to poor transfection efficiency of plasmid DNA and the better known islet transduction efficiency of adenoviral (Adv) vectors, in this study, we constructed Adv-hVEGF-hIL-1Ra by cloning hVEGF and hIL-1Ra coding sequences and polyA signal under separate cytomegalovirus (CMV) promoters in Adenoquick plasmid (Ad 13.1). There was dose and time dependent expression of these genes after transduction of Adv-hVEGF-hIL1Ra into human islets.

The mRNA expression of hVEGF and hIL-1Ra was more than 100 times higher than that of the non-transduced and bicistronic plasmid transfected control islets. Transduced islets were viable as evidenced by insulin release upon glucose challenge. Co-expression of hVEGF and hIL-1Ra by islets showed decrease in caspase- 3 activity and apoptosis induced by a cocktail of inflammatory cytokines such as TNF- $\alpha$, IL- $1 \beta$ and IFN- $\gamma$. Compared to non-treated or Adv-LacZ transduced islets, transduction of islets with AdvhVEGF-hIL-1Ra prior to transplantation under the kidney capsules of diabetic NODSCID mice reduced the blood glucose levels, and increased serum insulin and c-peptide levels. Immunohistochemical staining of the islet bearing kidney sections at day 30 after transplantation was positive for human insulin, hVEGF and von Willebrand factor.
\end{abstract}

Further, we decided to replace VEGF with hepatocyte growth factor (HGF) as it not only can promote revascularization of islets but also increases $\beta$-cell proliferation. It can also protect the islets from apoptosis. Adv-hHGF-hIL-1Ra showed dose and time dependent expression of these genes after transduction into human islets and further showed significant decrease in caspase- 3 induced by the cytokines. Compared to untransduced islets, transduction of islets with Adv-hHGF-hIL-1Ra at $1000 \mathrm{MOI}$ prior to transplantation under the kidney capsules of streptozotocin-induced-diabetic NOD-SCID mice reduced blood glucose levels, and increased serum insulin and c-peptide levels.

These results indicate that the bicistronic Adv vector efficiently expresses both growth factor and antiapoptotic genes, decreases apoptosis and improves the outcome of islet transplantation.

Liver fibrosis is a consequence of chronic liver disorders which lead to accumulation of extra cellular matrix (ECM). Particularly, there is an increased accumulation of collagen in the fibrotic liver. We have therefore used a triplex forming oligonucleotide (TFO) against the type $\alpha 1$ (I) collagen and evaluated, whether it can attenuate liver fibrosis induced by common bile duct ligation (CBDL). There was a significant decrease in hydroxyproline levels in TFO-treated groups compared to non-treated CBDL group. Masson's trichrome staining showed weak staining for collagen in TFO-treated liver tissues and there was a predominantly high staining in non-treated CBDL group. There was over expression of $\alpha$-smooth muscle actin ( $\alpha$-SMA), marker for myofibroblasts following CBDL. Western blotting analysis also revealed an increase in TGF- $\beta 1$ 
expression, which is a potential marker for fibrosis in the CBDL group compared to TFO-treated group. These results suggest that TFO can be used to downregulate Type I collagen gene expression and can alleviate liver fibrosis induced by common bile duct ligation. 


\section{TABLE OF CONTENTS}

CHAPTER 1. GENE THERAPY AND ISLET TRANSPLANTATION ...................1

1.1. Introduction.................................................................................................. 1

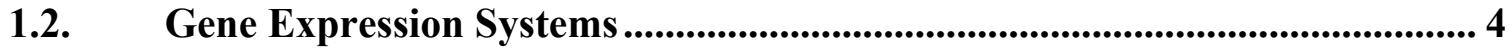

1.2.1. Basic Components of Plasmid Vectors............................................4

1.2.2. Persistence of Gene Expression .......................................................9

1.2.3. Site-Specific Gene Expression........................................................ 13

1.2.4. Pulsatile Gene Expression................................................................. 14

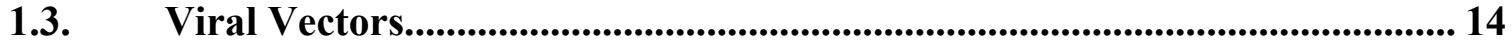

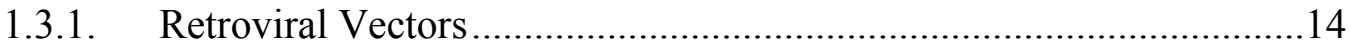

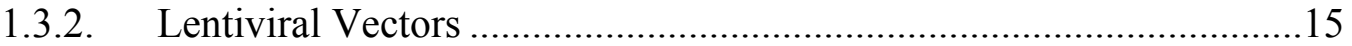

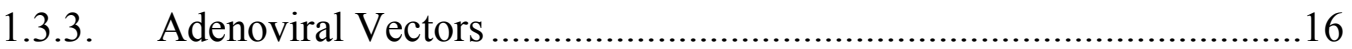

1.3.4. Adeno-Associated Viral Vector ........................................................ 18

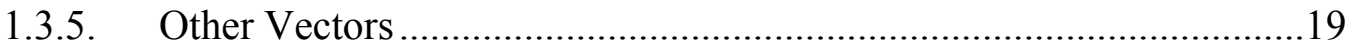

1.4. Gene Delivery Systems ................................................................................ 19

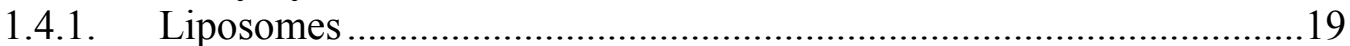

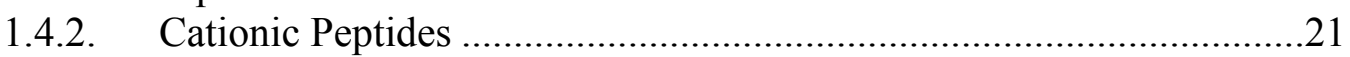

1.4.3. Cationic Polymers and Lipopolymers...........................................22

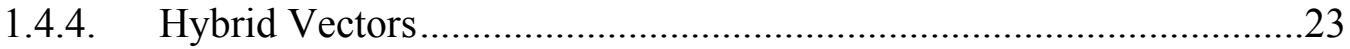

1.4.5. Receptor-Mediated Gene Transfer...............................................24

1.5. Concluding Remarks ............................................................................................. 24

CHAPTER 2. BIPARTITE VECTOR ENCODING hVEGF AND hIL-1Ra FOR EX VIVO TRANSDUCTION INTO HUMAN ISLETS ..................................26

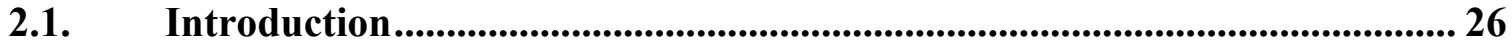

2.2. Materials and Methods................................................................................. 27

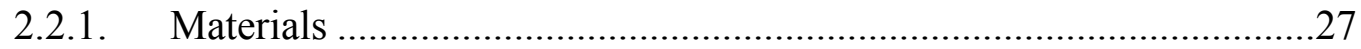

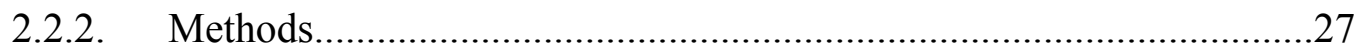

2.2.3. Construction of Adv-hVEGF-hIL-1Ra ............................................27

2.2.4. Determination of Viral Titer ...............................................................28

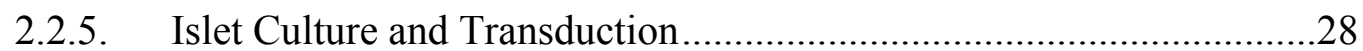

2.2.6. Quantitative Real Time PCR .........................................................28

2.2.7. In Vitro Islet Function after Transduction ..........................................29

2.2.8. Cytoprotective Effect of Adv-hVEGF-hIL-1Ra .............................29

2.2.9. Islet Transplantation Studies..............................................................30

2.2.10. Immunohistochemistry and Morphometric Analysis..........................30

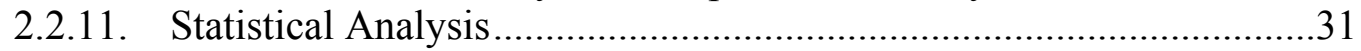

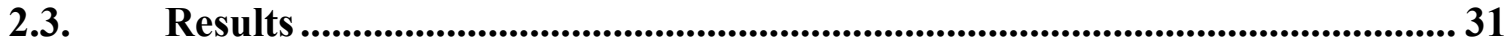

2.3.1. Construction of Adv-hVEGF-hIL-1Ra ........................................... 31

2.3.2. Transduction Efficiency of Adv-hVEGF-hIL-1Ra .............................31

2.3.3. Effect of Viral Transduction on Islet Function ......................................34

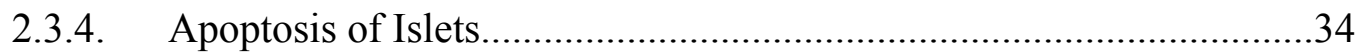

2.3.5. Islet Survival and Function after Transplantation..............................38

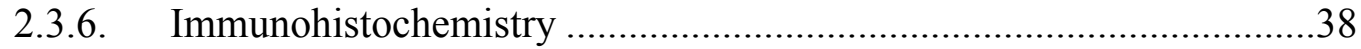

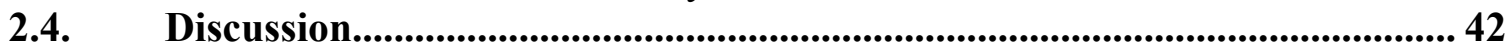


CHAPTER 3. BIPARTITE ADENOVIRAL VECTOR ENCODING hHGF AND hIL-1Ra FOR IMPROVED HUMAN ISLET TRANSPLANATATON...........46

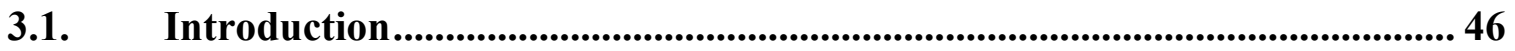

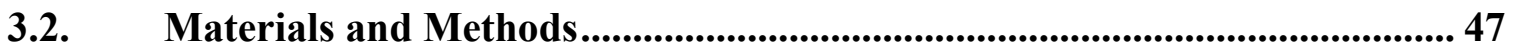

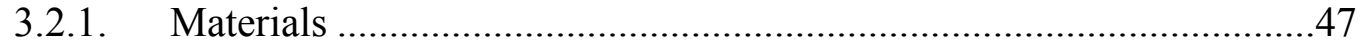

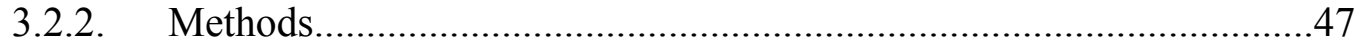

3.2.3. Construction of Adv-hHGF-hIL-1Ra .............................................47

3.2.4. Determination of Viral Titer ..........................................................48

3.2.5. Islet Culture and Transduction........................................................48

3.2.6. Quantitative Real Time PCR ......................................................48

3.2.7. In Vitro Islet Function after Infection ................................................49

3.2.8. Cytoprotective Effect of Adv-hHGF-IL-1Ra...................................49

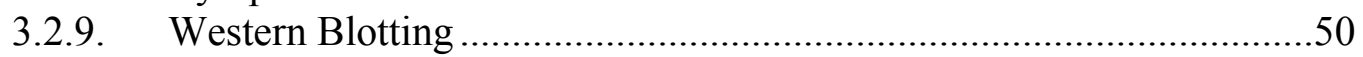

3.2.10. Islet Transplantation Studies .......................................................50

3.2.11. Intraperitoneal Glucose Tolerance Test ........................................50

3.2.12. Immunohistochemistry and Morphometric Analysis........................51

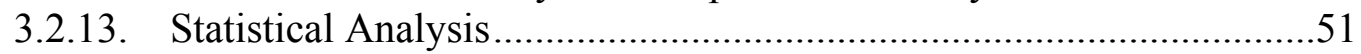

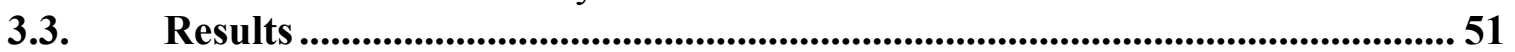

3.3.1. Construction of Adv-hHGF-hIL-1Ra ...........................................51

3.3.2. Transduction Efficiency of Adv-hHGF-hIL-1Ra ............................51

3.3.3. Effect of Viral Transduction on Islet Function ...................................54

3.3.4. Apoptosis of Islets...................................................................54

3.3.5. Islet Survival and Function after Transplantation.............................58

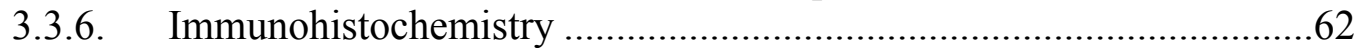

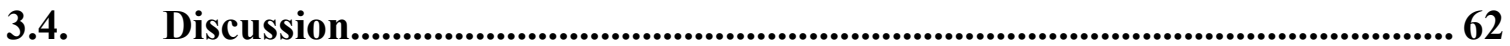

\begin{tabular}{|c|c|c|}
\hline $\begin{array}{l}\text { IY } \\
\text { INI }\end{array}$ & $\begin{array}{l}\text { LR 4. } \\
\text { LPHA } \\
\text { D BY }\end{array}$ & 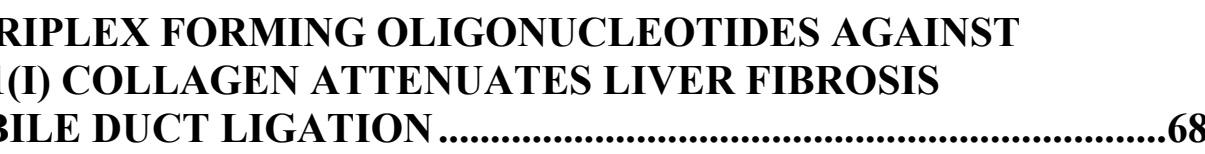 \\
\hline 4.1. & Introd & ction \\
\hline 2. & Mater & 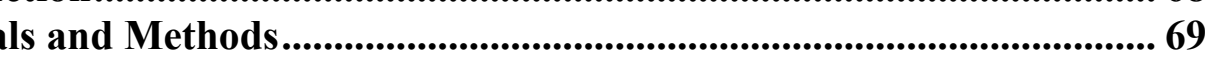 \\
\hline & 4.2.1. & 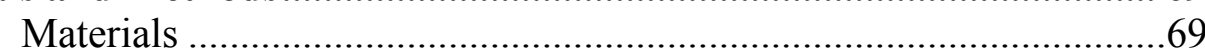 \\
\hline & 4.2.2. & Animals and Experimental Design .......... \\
\hline & 4.2.3. & Cell Culture.... \\
\hline & 4.2.4. & 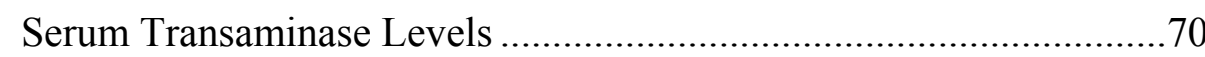 \\
\hline & 4.2 .5 . & 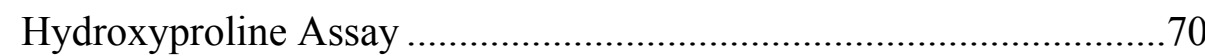 \\
\hline & 4.2.6. & 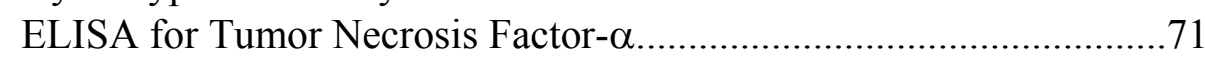 \\
\hline & 4.2.7. & Myeloperoxidase (MPO) Activity Assay ........................................ 71 \\
\hline & 4.2.8. & Real Time Polymerase Chain Reaction ................ \\
\hline & 4.2.9. & Histological Staining... \\
\hline & 4.2 .10 & Immunofluorescent Staining \\
\hline & 4.2 .11 & Statistical Analysis... \\
\hline
\end{tabular}

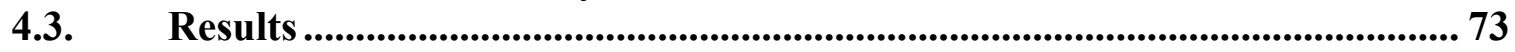

4.3.1. TFO Downregulates Type 1 (I) $\alpha$-Collagen In Vitro ..........................73 
4.3.2. TFO Downregulates Profibrogenic and Proinflammatory Cytokines in HSCs In Vitro ...

4.3.3. TFO Attenuates Chronic Liver Injury in CBDL Rats...........................75

4.3.4. Histopathological Alterations ..............................................................75

4.3.5. Effect of TFO on Liver Functions and Serum Fibrotic Markers ..........80

4.3.6. TFO Attenuates In Vivo Myofibroblast Activation ..............................80

4.3.7. TFO Inhibits Neutrophil Infiltration by Downregulating Inflammatory Cytokines ...................................................................86

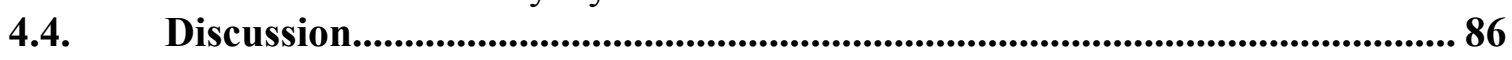

CHAPTER 5. SUMMARY..............................................................................................89

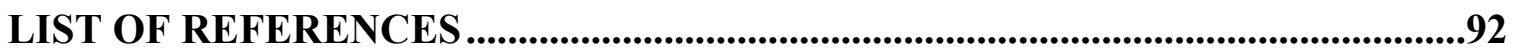

VITA........................................................................................................................104 


\section{LIST OF FIGURES}

Figure 2-1 Construction of E1 and E3 deleted Adv-hVEGF-hIL-1Ra............32

Figure 2-2 Time profile of hVEGF and hIL-1Ra at protein levels..............33

Figure 2-3 Real time PCR of islets transduced with Adv-hVEGF-IL-1Ra........35

Figure 2-4 Glucose Stimulation Index..................................

Figure 2-5 Effect of Adv-hVEGF-hIL-1Ra expression on caspase-3 activity..... 37

Figure 2-6 Effect of hVEGF and hIL-1Ra co-expression on islet viability....... 39

Figure 2-7 Transplantation of Adv-hVEGF-hIL-1Ra transduced islets.........40

Figure 2-8 Immunohistochemical staining of islet bearing kidney sections...... 41

Figure 3-1 Construction of E1 and E3 deleted bipartite Adv-hHGF-hIL-1Ra.....52

Figure 3-2 Expression of HGF and IL-1Ra genes at protein level...............53

Figure 3-3 Real time PCR of Adv-hHGF-hIL-1Ra transduced human islets..... 55

Figure 3-4 Glucose Stimulation Index....................................56

Figure 3-5 Effect of hHGF and hIL-1Ra co-expression on caspase-3 activity.... 57

Figure 3-6 Effect of Adv-hHGF-hIL-1Ra on Bcl-2 and Bax protein levels.......59

Figure 3-7 Transplantation of Adv-hHGF-hIL-1Ra transduced islets........... 60

Figure 3-8 Intraperitoneal Glucose Tolerance Test.........................6 61

Figure $3-9 \quad$ Serum insulin and c-peptide ELISA ........................... 63

Figure 3-10 Immunohistochemical staining of islet bearing kidney sections......6 64

Figure 4-1 TFO down regulated type 1 (I) $\alpha$-collagen in HSCs..................74

Figure 4-2 In vitro effect of TFO on HSC cells...........................

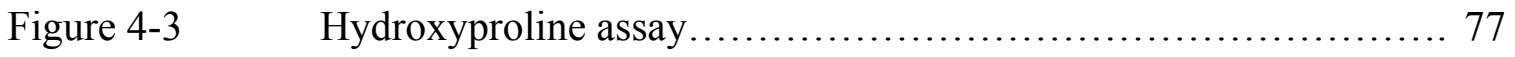

Figure 4-4 Hematoxylin and Eosin staining of livers sections...................78 
Figure 4-5 Masson's Trichrome staining for collagen...................... 79

Figure 4-6 Serum ALT and AST assay................................. 81

Figure 4-7 Real time PCR analysis for $\alpha$-SMA......................... 82

Figure 4-8 Immunoflorescent staining for $\alpha$-SMA....................... 83

Figure 4-9 Double immunostaining for cytokeratin-19 and $\alpha$-SMA............84

Figure 4-10 Colocalization of Cytokeratin-19 and $\alpha$-SMA....................85

Figure $4-11 \quad$ TNF- $\alpha$ ELISA and Myeloperoxidase assay $\ldots \ldots \ldots \ldots \ldots \ldots \ldots \ldots . \ldots 7$ 


\section{LIST OF ABBREVIATIONS}

\begin{tabular}{|c|c|}
\hline$\alpha-\mathrm{SMA}$ & Alpha-Smooth Muscle Actin \\
\hline APC & Antigen Presenting Cell \\
\hline Adv & Adenoviral Vector \\
\hline BSA & Bovine Serum Albumin \\
\hline CAR & Coxsackievirus-Adenovirus Receptor \\
\hline $\mathrm{CAG}$ & CMV Early Enhancer/Chicken $\beta$ Actin Promoter \\
\hline CMV & Cytomegalovirus \\
\hline CMRL & Connaught Medical Research Laboratories (medium) \\
\hline CTL & Cytotoxic T-Lymphocytes \\
\hline CTLA-4 & Cytotoxic T-Lymphocyte antigen-4 \\
\hline DOSPER & 1, 3-Di-Oleoyloxy-2-(6-Carboxy-spermyl)-Propylamide \\
\hline DOTAP & 1, 2-Dioleoyl-3-trimethylammonium-propane \\
\hline ECM & Extra Cellular Matrix \\
\hline ECMV & Encephalomyocarditis Virus \\
\hline EF-1 $1 \alpha$ & Elongation Factor-1Alpha \\
\hline EGFP & Enhanced Green Fluorescent Protein \\
\hline EMT & Epithelial Mesenchymal Transition \\
\hline ES & Embryonic Stem (Cells) \\
\hline FITC & Fluorescein Isothiocyanate \\
\hline GST-1 & Glutathione S-Transferase \\
\hline HGF & Hepatocyte Growth Factor \\
\hline HSA & Human Serum Albumin \\
\hline HUVEC & Human Umblical Vascular Endothelial Cells \\
\hline IDDM & Insulin Dependent Diabetes Mellitus \\
\hline $\mathrm{IE}$ & Islet Equivalents \\
\hline $\mathrm{IFN} \gamma$ & Interferon- $\gamma$ \\
\hline $\operatorname{Ig}$ & Immunoglobulin \\
\hline IGF & Insulin-like Growth Factor \\
\hline IL-1 $\beta$ & Interleukin-1 $\beta$ \\
\hline IL-1Ra & Interleukin-1 Receptor Antagonist \\
\hline iNOS & Inducible Nitric Oxide Synthase \\
\hline IRES & Internal Ribosome Entry Site \\
\hline LTR & Long Terminal Repeats \\
\hline $\mathrm{MHC}$ & Major Histocompatibility Complex \\
\hline $\mathrm{mPEG}$ & Monomethoxy Poly(Ethylene Glycol) \\
\hline MPO & MyeloPeroxide Assay \\
\hline NIDDM & Non-Insulin Dependent Diabetes Mellitus \\
\hline NO & Nitric Oxide \\
\hline NOD & Non-Obese Diabetic \\
\hline pDNA & Plasmid DNA \\
\hline PDX-1 & Pancreatic and Duodenal Homeobox Gene 1 \\
\hline PEG & Poly (Ethylene Glycol) \\
\hline PLL & Poly (L-Lysine) \\
\hline
\end{tabular}


PVA

PVP

RIA

SCID

SV 40

TCR

Tet

TFO

TGF $\beta$

$\mathrm{TNF} \alpha$

TRE

VEGF

vWF
Poly (Vinyl Alcohol)

Poly (Vinyl Pyrrolidone)

RadioImmunoAssay

Severe Combined Immunodeficient

Simian Virus 40

T-cell Receptor

Tetracycline

Triplex Forming Oligonucleotide

Transforming Growth Factor- $\beta$

Tumor Necrosis Factor- $\alpha$

Transcription Regulatory Elements

Vascular Endothelial Growth Factor

von Willebrand Factor 


\section{CHAPTER 1. GENE THERAPY AND ISLET TRANSPLANTATION}

\subsection{Introduction}

Diabetes mellitus is a chronic metabolic disorder affecting millions of individuals and is purported to increase inexorably in the coming decades. It is usually a consequence of environmental or genetic factors resulting in elevated blood glucose levels. It is generally categorized into two types. Type I or juvenile onset diabetes mellitus is more common in children due to failure of islets to secrete insulin. Type II diabetes mellitus is more prevalent in adults wherein the amount of insulin produced is not sufficient to control the blood glucose levels. It can be a result of insulin resistance or decrease in insulin secretion by the beta cells. There is no proper cure for diabetes. Insulin is the only treatment for Type I diabetes patients and most existing therapies often fail to provide adequate control. There is an urgent need for an effective and better alternative for managing diabetes and ultimately a cure.

In Type I diabetes, there is a near depletion of the beta $(\beta)$-cell mass, suggesting the need for $\beta$-cell replacement. ${ }^{1,2}$ There is also a reduction in the $\beta$-cell mass in Type I or II diabetes or both. To date, the most effective way of regulating glucose levels is by administration of exogenous insulin. However, several studies have shown that insulin administration does not decrease the risk of diabetic complications, and hypoglycemia is a potential drawback of insulin therapy. This problem is more severe in patients with erratic glucose levels or hypoglycemic unawareness. In this scenario, whole organ pancreas transplantation seems ideal and it can completely restore the patients to euglycemia and maintain long term graft survival. However, the major drawbacks with pancreas transplantation are the complications that occur with the major surgery required to perform this procedure and the use of immunosuppressants. There are instances where simultaneous pancreas kidney transplants have been found beneficial leading to longterm survival. ${ }^{3}$ However, pancreas transplantation does not overcome the diabetic complications hyperglycemia, hypoglycemia and microvascular and macrovascular complications, which are a major concern. ${ }^{4}$ Also, pancreas transplantation requires major surgery and lifelong immunosuppression.

Since the advent of Edmonton protocol in 2000, pancreatic islet transplantation has also become a viable alternative for Type I diabetic patients having life threatening hypoglycemic episodes. There is a decrease in hypoglycemia and sustained function of islets after successful islet transplantation. However, patients becoming insulin independent usually lasts for a few years after which they require administration of insulin. The major concern with islet transplantation is the use of immunosuppressant's to counteract the immune responses elicited from both the host and the transplanted islets. The other major problem is the primary non-function of islets due to hypoxia resulting from lack of revascularization or immune mediated destruction of transplanted islets.

In 2000, it was reported that seven consecutive subjects with Type I diabetes were rendered insulin free following islet transplantation and according to recent reports $72 \%$ 
of them are insulin independent. The important achievement of this procedure was the lack of major surgery for transplanting islets and elimination of hypoglycemia which was an important complication in pancreas transplantation or any other therapy for Type I diabetes. The other major outcome of this study was that a number of recipients became insulin dependent following administration of islets from one donor. However, more than $50 \%$ of the patients who became insulin independent needed insulin within 2 years. There was complete graft failure within a few years. This can be attributed largely to continuing graft assault by autoimmunity as well as toxicity from immunosuppressive drugs.

Islet transplantation can be done at various sites; however the problems associated with transplantation need to be overcome to prolong the survival and metabolic outcome of islets. Autologous or auto-transplantation refers to the transplantation of islets isolated from the same animal; from same species as allogenic or allo-transplantation and from different species as xenogenic or xenotransplantation.

There are several factors that cause the primary non-function of the islet graft. Islet primary non-function is the failure of islets to function immediately after transplantation due to reasons other than graft failure. During isolation and transplantation islets get subjected to different conditions including mechanical stress that may result in nonfunction of the $\beta$-cells. Several studies have shown that islets fail to function after a period of time following transplantation either in the pancreatic cavity or in the peritoneal cavity. ${ }^{5,6}$ There was a significant depletion of islet mass indicating that primary nonfunction is one of the major reasons we need a large number of islets to achieve euglycemia. Efficient isolation of pure islets, without altering their physical appearance is vital for successful transplantation. Islets are the endocrine part of the pancreas and they need to be separated from the exocrine cells while keeping the intra-islet association intact. The enzyme collagenase is used to disrupt the islet cells interaction with exocrine cells followed by separation using density gradient centrifugation. The whole process has been optimized and pure, viable islets are being successfully isolated.

Islets have extensive intra-islet vasculature which gets disrupted during their isolation. This vasculature is essential for the supply of nutrients and oxygen to the islets. Since this vasculature gets disrupted during isolation, revascularization is very essential for the viability and function of islets following transplantation. Islets get revascularized by secreting pro-angiogenesis molecular mediators like vascular endothelial growth factor (VEGF) and its receptors. Therefore, ex-vivo gene delivery of VEGF to islets or by co-encapsulating VEGF protein with islets can promote revascularization in islets.

Type I diabetes is an autoimmune disorder which poses several challenges for islet transplantation. Diabetes shows the presence of $\beta$-cell reactive auto antibodies and Tcells. The specific nature of T-cells is not known and these can elicit an autoimmune reaction following transplantation resulting in the destruction of $\beta$-cells. The host also has preexisting antibodies and primed immune cells against $\beta$-cell surface epitopes and immune cells that infiltrate in response to non-self antigens resulting in graft destruction. Apart from this, the host also reacts to non-self proteins from the transplant tissue resulting in the loss of islet function. 
Usually when a foreign tissue is introduced into the host by transplantation, foreign antigens are recognized by the host leading to a cascade of events called antigen presentation. The various peptide fragments, secreted and shed proteins come in contact with the host immune cells forming a major histocompatibility complex (MHC). ${ }^{7} \mathrm{MHC}$ can be either class I or II and usually autoimmunity occurs when there is matching for MHC class II. ${ }^{8}$ Class II molecules are only expressed in certain immune cells including macrophages and dendritic cells known as antigen presenting cells (APCs). APCs can be either from the host or from the donor. The antigens present on MHC II molecules on the donor APCs acts as foreign antigens to the transplant recipient when the lymphocytes migrate in the host. Donor antigen presentation can be mediated either through direct or indirect pathways. Direct pathway engages antigen presentation by the donor APCs, while the indirect pathway engages host APCs. Understanding the pathways involved in immune destruction of transplanted islets is critical for developing strategies to protect them.

Various immune suppressive strategies are employed to minimize or block the immune activation. Immunosuppressive drugs are often employed with transplantation. However, the risks from these drugs are unknown and they may further inhibit the graft function. Therefore, there is a need for improvements in immunosupprresion and also improvement in the quality of islets.

The potential use of genes as therapeutics has attracted great attention for the treatment of severe and debilitating diseases. Gene therapy is the method of treating diseases using genes so that the patient's somatic cells can produce the specific proteins that are lacking. This will prevent the limitations associated with the administration of therapeutic proteins. It is an approach to treat diseases, or ultimately prevent a disease by replacing defective genes, introducing new genes, or changing endogenous expression of genes. Gene therapy can be used to express genes that can aid in the process of islet transplantation and improve the overall survival and function of islets. It can aid in modifying the islets resulting in improved survival and function of islets posttransplantation. Current gene therapy approaches are mainly oriented to improve islet function, block apoptosis and inhibit primary non-function of islets. The various gene vectors that are being used for islet transplantation include adenoviral, adeno-associated virus, herplex simplex virus vectors, retro and lentiviral vectors and non-viral vectors. The main problem associated with viral vectors is immunogenicity and safety concerns. Anti-apoptotic, angiogenic genes are most commonly expressed using these vectors to improve the metabolic function and survival of islets following transplantation.

Gene therapy uses viral or non-viral vectors to deliver genes to synthesize specific therapeutic proteins. Viral vectors are derived from retroviruses, lentiviruses, adenoviruses, adeno-associated viruses, herpes viruses and pox viruses. ${ }^{9}$ The development of vectors for cell-specific gene expression is the major goal of any gene therapeutic strategies. Significant progress has been made in the construction of gene expression vectors that combine different functions required for efficient gene transfer. ${ }^{10}$

Gene medicine usually contains an expression system that controls the transcription 
of a gene within the target cell and a specific delivery system that controls the biodistribution of these vectors to specific locations in the body. Cationic liposomes, peptides and polymers are commonly used as transfection reagents for gene expression plasmids, while viral vectors usually do not require any of these transfection reagents.

Viral vectors have shown much promise in the field of gene therapy, but there are safety issues, they may also be limited in terms of their DNA loading capacities. ${ }^{10}$ Immune activation by viral vectors is a major concern as many of the vectors with high therapeutic activity in vitro fail to do so in vivo. ${ }^{11}$ Moreover, increasing their yield can be quite difficult. To minimize their immunogenicity, the surface of the viral vectors is often modified by conjugating to polyethylene glycol (PEG). ${ }^{12}$

Gene expression plasmids offer many advantages over viral vectors, including large packaging capacity, no integration into the host genome, and lower toxicity. However, their level of gene expression is often lower and shorter than that of the viral vectors. Recent advances in gene expression systems have shown great improvement in the transfection levels of non-viral vectors. Plasmid vectors can also be used to silence a gene, thereby causing the inhibition of an abnormal protein production in the body. ${ }^{13}$ Plasmids have also shown to play a role in the formation of viral vectors, e.g., two plasmid rescue systems used to construct an adenoviral vector. The various parts of the gene expression system are outlined here.

\subsection{Gene Expression Systems}

Gene expression systems are broadly classified into plasmid and viral vectors. Basically, these vectors have the machinery in them to facilitate the production of target proteins in the host upon transfection or transduction.

\subsubsection{Basic Components of Plasmid Vectors}

A gene expression plasmid contains a complementary DNA (cDNA) sequence coding for either a full gene or minigene, and several other genetic elements including promoter, introns, polyadenylation (polyA) sequences, and transcript stabilizers to control the transcription, translation, and protein stability and/or secretion from the host cell. ${ }^{14}$

The transcription unit comprises of 5' enhancer/promoter upstream of the gene encoding a therapeutic protein and polyA signal downstream of the gene. An intron is also assembled into either 5' or 3' untranslated region (UTR), leading to elevation of mRNA levels. Plasmid vectors can be designed to express two genes simultaneously driven by different promoters or a single promoter or by insertion of an internal ribosome entry site (IRES). IRES was first discovered in picornaviruses, Encephalomyocarditis Virus (ECMV) and poliovirus. ${ }^{15,16}$ They have the ability to translate two open reading frames from a single mRNA transcript. Usually the IRES is inserted between two transgenes to be expressed. IRES confers unequal expression of genes and usually the 
expression of the downstream gene is relatively higher than the upstream gene and this may confirm the internal ribosome entry. ${ }^{16}$ However, recent studies have shown that this may not be always true. Production of low level of monocistronic RNAs putatively due to splicing of the bicistronic transcript due to the presence of 3' splice site (SS) which can show increased expression of the downstream gene. ${ }^{17}$ This warrants a careful RNA structural analysis to confirm the functionality of the IRES.

We have constructed a bicistronic plasmid vector (phVEGF-hIL-1 Ra) encoding human vascular endothelial growth factor (hVEGF) and human interleukin-1 receptor antagonist (hIL-1Ra) driven by cytomegalovirus (CMV) and elongation factor-1 $\alpha$ (EF$1 \alpha)$ promoters, respectively, in pBudCE4.1 vector. ${ }^{18}$ There was dose and time dependent expression of hVEGF and hIL-1Ra genes when they were transfected into human islets. However, the expression levels of these two genes were not sufficient to decrease blood glucose levels of diabetic mice following transplantation with phVEGF-hIL-1Ra transfected islets. Not only the expression, but the extent of expression of genes was also low for this bicistronic plasmid.

\subsubsection{Bacterial Elements}

Plasmids encode two features that are important for their propagation in bacteria. One is the bacterial origin of replication (Ori), which is a specific DNA sequence that binds to factors that regulate replication of plasmid and in turn control the number of copies of plasmid per bacterium. The second required element is a selectable marker, usually a gene that confers resistance to an antibiotic. The marker helps in the selection of bacteria that have the gene expression plasmid of interest. Escherichia coli (E.coli) are commonly used bacteria for propagating plasmids. It has the property to transfer DNA either by bacterial conjugation, transduction or transformation. The extensive knowledge about E.coli's physiology and genetics accounts for its preferential use as a host for gene expression. Human insulin was the first product to be produced using recombinant DNA technology from E.coli.

\subsubsection{Transcription Regulatory Elements (TRE)}

Gene expressing plasmids contain transcription regulatory elements (TRE) to control transcription. The Jacob and Monad theory postulates that a repressor protein may bind to the operator region downstream of a promoter preventing RNA polymerase from binding to the host DNA. ${ }^{19}$ This operator region may overlap with the promoter for the operon being controlled. However, if this repressor is controlled, it may increase gene expression. ${ }^{20}$ Transcription regulatory elements play a significant role in gene expression and may also impart specificity. The important sequences in the gene that control transcription are the cis-acting sequences that are situated in the immediate vicinity of the gene and they together constitute the functional unit or domain. ${ }^{21}$

Heterologous TREs can be included in the adenoviral genome to allow replication only in the cells in which TREs are functional, resulting in cell specificity. ${ }^{22}$ Addition of new TREs in an expression vector can alter the transcription of the target gene. These 
new elements can mimic the action of the genomic TREs of the target genes resulting in an alteration in transcription. These elements can be added to either increase or decrease transcription depending on the requirement. In diseases like cancer, the expression of target genes can be altered by this method. ${ }^{23}$ For example, homeodomain-containing transcription factor pancreatic duodenal homeobox-1 (Pdx-1) plays a key role in maintaining the function of pancreas and is also known to have a prominent role in beta cell development. ${ }^{24}$ When this Pdx-1 was introduced into an adenoviral vector and expressed in hepatocytes, they were transformed into pancreatic endocrine hormone producing cells. ${ }^{25}$ Modulating the expression vector with TREs can be done to increase the gene expression. Embryonic stem cells have the potential to transform into insulinproducing cells that can be transplanted for treating Type I diabetes. ${ }^{26}$ TREs can play a major role in transformation of these cells.

\subsubsection{Enhancer}

Enhancer is a short region of DNA that can bind trans-acting factors, much like a set of transcription factors, to enhance transcription levels of genes in a gene-cluster. Usually in the bound proteins facilitate promoter-binding proteins to interact with the promoter. ${ }^{27}$ An enhancer does not need to be particularly close to the genes it acts on, and need not be located on the same chromosome. An enhancer does not need to bind close to the transcription initiation site to affect its transcription, as some have been found to bind several hundred thousand base pairs upstream or downstream of the start site. ${ }^{28}$ Enhancers can also be found within introns. An enhancer's orientation may even be reversed without affecting its function. Furthermore, an enhancer may be excised and inserted elsewhere in the chromosome, and still affect gene transcription. ${ }^{29}$ That is the reason why intron polymorphisms are important, even though they are not transcribed and translated. Enhancer-promoter interaction also plays a major role in immune reaction following in vivo administration of plasmid vectors. Epo enhancer when inserted into the plasmid expressing VEGF showed expression of VEGF in hypoxia conditions, whereas the plasmid without epo enhancer showed no expression of VEGF. ${ }^{30}$

\subsubsection{Promoter}

Promoter is a DNA sequence that enables a gene to be transcribed. The promoter is recognized by RNA polymerase, which binds it and then initiates transcription.

Promoters regulate protein synthesis indirectly by having an active role in demarcating genes to be used for mRNA synthesis. The promoter region is usually the beginning of an operon, which is a collection of neighboring genes, and controls the region transcribed into the same mRNA molecule. ${ }^{31}$ Therefore, DNA transcription begins after RNA polymerase has bound downstream of the therapeutic gene. This effect is seen as DNA unwinding into single strands. Therefore, any mutation in this region will prevent RNA polymerase from binding. Transcription begins at the first base of the target gene +1 position is the TATAA sequence or TATA box, 5' TATA (A/T) A (A/T)-3'. This region ensures that transcription starts at the proper point and binds the RNA polymerase II

complex. ${ }^{32}$ Another transcription start site is the CCAAT box (consensus sequences 5'GGTC-CAATCT-3') located upstream of the TATA box. 
Promoter sequences play an important role not only in initializing gene transcription, but also in immunostimulation..$^{33}$ The promoter type governs the strength and duration of transgene expression. Viral promoter elements are first known to be used in an expression vector to express proteins for gene therapy. Cytomegalovirus (CMV), Rous sarcoma virus (RSV) and Simian virus 40 (SV40) are some of the strongest known viral promoters. However, there are some drawbacks with the use of viral promoters such as, lack of specificity and immunostimulation that results in inactivation. For example, CMV promoter shows expression in most cell types, however, its activity decreases over a period of 3-4 weeks. ${ }^{34}$ This is possibly due to the inhibition by cytokines, methylation, or inactivation by repressor proteins. This is also true for other viral promoters like SV 40 and RSV. ${ }^{35}$ Because of these reasons there is an urgent need to develop promoters based on non-viral cellular regulatory elements.

Sustained gene expression is quite difficult to achieve. However, some promoters have been reported to confer sustained gene expression from plasmid DNA in vivo. These promoters include $\beta$-actin, elongation factor $1-\alpha,(E F 1-\alpha)$, or ubiquitin. The activity of these promoters is usually lower than that of viral promoters, but can be increased by addition of viral or cellular enhancer components. ${ }^{36} \mathrm{CMV}$ early enhancer/chicken $\beta$ actin (CAG) is a promoter of this kind. It consists of CMV enhancer and first intron of chicken (or human skeletal) $\beta$-actin. It shows activity similar to or more than the CMV promoter. ${ }^{37,38}$ It shows a greater activity in viral vectors, but its expression profile needs to be established for using in plasmid vectors. ${ }^{35}$

EF1- $\alpha$ promoter has been reported to confer sustained gene expression compared to CMV promoter, but its expression is 10 -fold lower than the CMV promoter. ${ }^{39}$ Promoters from three of the known human ubiquitin genes, UBA, UBC, UBB have been incorporated in plasmid vectors. Luciferase gene expression was measurable upto 6 months when the plasmid contained UBC promoter. ${ }^{39}$ This clearly suggests that if proper promoters are incorporated into the gene expression system, it is possible to attain sustained gene expression. Primary bone marrow-derived mesenchymal stromal cells (BMMSC) were inserted with glucose-responsive promoter-regulated insulin transgene. HBMMSC glucose-responsive promoter, EGR1 was identified from these cells which showed rapid glucose-induced transgene secretions simulating normal human islets. ${ }^{40}$

\subsubsection{Untranslated Region (UTR)}

The 5' untranslated region (5'-UTR) is the region of the mRNA transcript that is located between the capsite and the initiation codon. The linkage between methylated $G$ residue and 5' to 5' triphosphate bridge is known as the cap structure, which is essential for efficient initiation of protein synthesis. The 5'-UTR is known to influence mRNA translation efficiency. In eukaryotic cells, initiation factors first interact with the 5' cap structure and prepare the mRNA by unwinding its secondary structure. An efficient 5'UTR is usually moderate in length, devoid of secondary structure and upstream initiation codons. It has AUG with an optimal context. Any of the following features that influence the accessibility of the 5' cap structure to initiation factors will influence mRNA translatability. ${ }^{41,42}$ 
Initiation codon AUG appears to be the best recognized when it is in the context of the sequence CCRCCAUGG with purine (R) at -3 and/or guanine $(\mathrm{G})$ at +4 (A of AUG is numbered+1). If an AUG occurs alone or in conjunction with a short open reading frame located between the cap site and the genuine AUG, translation will be inhibited. Secondary structures of the UTRs inhibit translation. 5' UTR lengths that are greater than 32, but less than 100 nucleotides, permit efficient recognition of the first AUG. Most naturally occurring 5' UTRs are 50-100 nucleotides in length.

The 3' UTR comprises the mRNA sequence following the termination codon. It plays an important role in mRNA stability. ${ }^{31}$ AU rich motifs are commonly found in the 3 ' UTR of mRNA of cytokines, growth factors, and oncogenes. These are mRNA instability elements and should be removed for maximal gene expression. This is achieved when standard 3' UTR sequence is used in the place of one found in cDNA. Another way is to minimize the length of the 3' UTR by placing the hexanucleotide of the poly A signal immediately downstream of the stop codon. ${ }^{43}$

\subsubsection{Polyadenylation (polyA) Signal}

The efficiency of polyadenylation (polyA) is important for gene expression, as transcripts that failed to be cleaved and polyadenylated are rapidly degraded in the nuclear compartment. ${ }^{44}$ PolyA signal is a recognition site consisting of AAUAAA hexamer positioned 10-30 nucleotides upstream of the 5' end and a GU or U rich element located maximally 30 nucleotides downstream of the 3 ' end. ${ }^{31}$ PolyA signal is needed for the formation of the 3' end of most eukaryotic mRNA. It directs two RNA processing reactions: site specific endonucleolytic cleavage of the RNA transcript, and stepwise addition of adenylates to newly generated 3' end to form the polyA tail.

\subsubsection{Intron}

The protein coding region in the gene is often interrupted by stretches of noncoding DNA called intron. Transcripts from intronless genes are rapidly degraded in the nuclear compartment, leading to lower gene expression. ${ }^{45}$ Therefore, for maximal gene expression in eukaryotic cells, at least one intron should be included within the transcription unit. Introns also promote mRNA export from the nucleus. The addition of intron A or B to the plasmid encoding ICAM-2 promoter and subsequent expression in HUVEC or PsCAM cells increased the expression of the transgene. ${ }^{37}$ Introns thus can affect gene expression and if used in a proper way can enhance the expression of transgenes.

\subsubsection{Stop Signal}

Stop signal is the DNA sequence at which RNA polymerase II is halted and detached from the DNA, thereby a stop or nonsense codon stops translation. However, polyA occurs at 5' AAUAAA 3' sequence in the mRNA. The polyA polymerase cleaves after the $\mathrm{U}$ residue and adds 50-250 adenylate residues. Stop signal not only plays an 
important role in gene regulation at the translational level allowing for rapid changes in specific protein levels, but also provides an opportunity to alter codon specificity.

\subsubsection{Multiple Cloning Sites (MCS)}

A multiple cloning site (MCS), also known as polylinker, is a short segment of DNA, which contains many restriction sites. Restriction sites within an MCS are unique and occur only once within a particular plasmid. MCS are commonly used for cloning of a single or multiple cDNAs due to its unique restriction endonuclease recognition sites identified with ease. The recombinant plasmid can be altered in such a way that the desired gene can be inserted into the plasmid and expressed. If there is no particular restriction site for a particular transgene, the restriction site can also be inserted into the MCS and then can be used to clone the gene in that vector.

\subsubsection{Fusion Tags}

Fusion tags are inserted in expression systems so that the DNA location and site specificity can be known. A variety of protein tags have been used to allow recombinant fusion protein to be detected and purified without the use of an antibody or other protein specific assays. Short epitope tags and fluorescent protein tags are commonly used for gene function studies. Epitope tags such as His/C-term, xpress, V5, FLAG, HA, and cMyc, circumvent the requirement for specific antibodies against target proteins. Fluorescent protein tags, such as green fluorescent protein (GFP) provides information on the cellular location of the fusion proteins. Since addition of the any amino acid residues may alter the properties of the target proteins, special consideration should be given on the intended use of the protein and on minimizing the adverse effects when picking a tag. Typically, a specific protease cleavage site is introduced in the tag and the target protein to facilitate tag removal if desired. Fusion tags can be incorporated into the plasmid vectors to enhance protein expression and solubility of the expressed protein. ${ }^{46}$ Fusion tags can be classified into two types based on their application: Affinity tags and solubility enhancing tags. Affinity tags help in purification of recombinant protein, whereas the solubility-enhancing tags can be used to improve the solubility of proteins. Usually the solubility enhancing tags are either large peptides or proteins. Fusion tags like glutathione S-transferase (GST) and maltose binding protein (MBP) show both affinity and solubility enhancing properties. ${ }^{47,48}$ Recombinant proteins often show large variability in their expression, solubility, stability, and function, making them difficult targets for large-scale analyses and production. Fusion tags can be very effective in improving the expression, solubility, and production of biologically active proteins, especially for proteins whose expression is very difficult.

\subsubsection{Persistence of Gene Expression}

Regulation of gene expression and having a sustained expression is the key for many diseases. Plasmid based systems usually confer transient gene expression, with less than

$20 \%$ of the peak level at day $3 .{ }^{49}$ This warrants the need for expression of transgene over 
a period of time. This is especially important in diseases like cancer where the cells divide continuously. An ideal non-viral vector should be able to provide persistent expression of the transgenes without affecting the host cells. Persistent gene expression can be achieved either by prevention of promoter attenuation, use of replicating plasmid, modulation of immune response, attachment of matrix/scaffold regions or optimizing plasmid size.

\subsubsection{Prevention of Promoter Attenuation}

The major drawback of non-viral vectors is the lack of sustained gene expression. Transient gene expression in vivo is partly due to promoter shutdown. ${ }^{35}$ This is especially true for viral promoters, such as CMV and SV 40 promoters. Methylation of plasmid DNA is involved in promoter inactivation, which can be prevented by removing the methylation sites (CpG motifs) from the plasmid construct. Transient gene expression is also due to the destruction of the transfected cells by the immune system. ${ }^{50}$ Viral promoters are sensitive to cytokines, which may explain the decline of their activity in vivo. ${ }^{35}$ Promoter inactivation has also been attributed to specific tissue types. For examples, smooth muscle gamma actin (SMGA) and flk-1 promoters show their activity specifically in smooth muscles and endothelium, respectively. ${ }^{10}$

\subsubsection{Use of Replicating Plasmids}

Extrachromosomally replicating vectors have great potential for use in gene therapy due to their high transfection levels and sustained expression of transgenes. The use of replicating plasmids results in sustained gene expression without the integration of gene into the host genome. This also reduces the risk of insertational mutagenesis. Replication elements usually from viral DNA are inserted into the expression systems that enable the plasmid to replicate extra chromosomally. This can be done by the introduction of a mammalian origin of replication ${ }^{51}$ sequence into plasmid. The best characterized oris in mammalian cells are from viral sources, such as SV40, human papo virus (BKV), bovine papilloma virus (BPV), and the Epstein Barr virus (EBV). ${ }^{52}$ Modeling the bacterial power and efficiency of self-replication would greatly increase therapeutic protein expression.

Since the plasmids do not integrate into the host genome, they reside outside as episomes. They have several advantages over integrating systems: (1) the transgene is not interrupted or subjected to regulatory constraints that often occur from integration into cellular DNA, (2) higher transfection efficiency, (3) episomes show a low mutation rate and tend not to rearrange, and (4) they have the ability to transfer large amounts of DNA. $^{10}$

Plasmids can replicate in both prokaryotic and eukaryotic cells provided they have certain elements which allow them to do that, e.g., EBV viral elements. ${ }^{53,54}$ Several viral constructs including EBV, BK virus (BKV), SV 40 and bovine papilloma virus have been used in constructing replicating plasmid vectors. Trans-acting factors are needed for the formation of episomal vectors, but there is a risk of transformation following their use. This is especially seen in the case of polyomaviruses (BKV, SV40) which contains a T- 
antigen (Tag) as a trans-acting factor. Tag purportedly binds to tumor suppressor gene p53 causing chromosmal aberrations and alters the gene expression. ${ }^{55}$ Considering this, EBV viral elements are safer as they have a low mutation frequency and can easily incorporate large amounts of DNA. ${ }^{55}$ EBV nuclear antigen 1 (EBNA1) and oriP constitute the EBV viral elements which impart stability to the viral DNA in the host cells. ${ }^{53,55}$ EBNA1 dimer/oriP complex controls the replication and transcription of plasmid vector. EBNA facilitates binding of the plasmid vector to the nuclear matrix. EBV-based vectors showed higher expression of transgenes compared to conventional vectors when they were studied in vivo. However, EBV based vectors are associated with certain drawbacks including integration into the host genome and oncogenicity. ${ }^{55}$ Ehrhardt et al., replaced the CMV promoter with a cellular promoter to minimize the silencing effects attributed to nonmammalian sequences. ${ }^{56}$ However, if the problems associated with the use of viral elements can be solved, they can be far superior compared to the conventional vectors.

\subsubsection{Modulation of Immune Response}

One of the major drawbacks of gene therapy is the interaction of the gene expression vectors with the host immune system. Although non-viral vectors produce less immune response compared to viral vectors, their effect should not be overlooked. CpG motifs are unmethylated with an ability to stimulate B cell proliferation, macrophage activation and maturation of dendritic cells. The bacterial and viral DNA contains a large amount of unmethylated $\mathrm{CpG}$ motifs. Plasmid vectors usually contain bacterial or viral elements with large number of $\mathrm{CpG}$ motifs, which lead to acute inflammatory response commonly seen in non-viral vectors. The sequence GTCGTT is the most active stimulatory CpG motif in humans leading to the activation of immune pathways. Following activation of the immune system by $\mathrm{CpG}$ motifs, gene expression is decreased due to cytokine mediated promoter shutdown and apoptosis of expressing cells.

When constructing plasmid vectors the immunostimulation of the $\mathrm{CpG}$ motifs can be reduced by methylation of the $\mathrm{CpG}$ motifs, using neutralizing $\mathrm{CpG}$ motifs or by elimination of $\mathrm{CpG}$ motifs. Plasmid vectors containing significantly less amount of $\mathrm{CpG}$ motifs showed very less immunostimulation compared to the ones with more $\mathrm{CpG}$ motifs. ${ }^{57}$ The presence of a single unmethylated $\mathrm{CpG}$ has been shown to elicit an inflammatory response when delivered to lung. However, $\mathrm{CpG}$ free vectors showed no inflammatory response and increased the duration of transgene expression in the lung. ${ }^{39}$ Li et al., have shown that intradermal injection of plasmid DNA encoding glutamic acid decarboxylase (GAD) polypeptide synthesized in both pancreatic islet and skin tissue and pro-apoptotic protein BAX, ameliorated new-onset Type I diabetes in NOD mice and increased skin allograft survival in a BALB/c-C57BL/6 model system in a donor-specific manner by preventing the autoimmune response that elicited following skin transplantation. ${ }^{58}$ Plasmid gene expression has resulted in expression of T-cells previously, whose effect can be negated by administering the plasmid DNA encoding this peptide. 


\subsubsection{Matrix/Scaffold Attachment Regions (MARs)}

Matrix/scaffold attachment regions (MARs) govern the architecture of the nucleus by establishing protein boundaries. Several proteins bind to MARs and these proteins are known as MAR binding proteins. MAR binding proteins are very significant as they regulate transcription, replication, repair and combination. ${ }^{59}$ Incorporation of matrix attachment regions in the plasmid construct may be thus helpful in maintaining episomal plasmid for extended periods. The potentially immunostimulatory and transforming properties of viral protein used in episomes and other components of the expression system impede the application of these systems for gene therapy. Alternatively MARS can be incorporated into circular plasmids to replace transacting viral gene products. MARs have been characterized as AT rich sequences generally composed of 4-6 bp motifs such as ATTA, ATTTA, or ATTTTA. ${ }^{60}$ These sequences are commonly used with gene enhancers and the ori. Incorporation of these sequences has been used to enhance the transcriptional activity of integrated transgenes. ${ }^{60}$

MARs are often situated in proximity to promoters, replication origins and other important regions in the genome. MARs have been known to play a role in several biological activities due to their affinity to the nuclear matrix. They have been known to play a role in initiation of transcription, promote long term expression of the transcript by counteracting the effects of DNA methylation, protect transgenes from negative effects of the genomic surroundings and promote histone acetylation, act as an enhancer and promote replication. ${ }^{61}$ MARs have been incorporated in episomally replicating plasmids to impart stability to the vector and increase the longevity of transgene expression. MARs are used to replace the viral elements from these vectors to minimize the immunostimulatory effects from them. An episomal vector was developed by replacing the Tag protein with MAR from human $\beta$-interferon gene while retaining the SV40 ori sequence. This vector replicated episomally in $\mathrm{CHO}$ cells and showed stable expression for more than 100 divisions. Argyros et al., ${ }^{62}$ demonstrated persistent transgene expression in the liver after hydrodynamic injection of the plasmid containing MARs driven by human liver specific promoter $\alpha 1$-anti-trypsin (AAT). MARs protected the AAT promoter by inhibiting methylation of $\mathrm{CpG}$ motifs, resulting in sustained expression of luciferase, ${ }^{62}$ while the plasmid containing MARs and CMV promoter did not show persistent luciferase expression. This signifies that even though MARs imparts extrachromosomal stability, duration of gene expression also depends on the choice of promoter used.

\subsubsection{Plasmid Size}

Plasmid stability is dependent on its size. Most gene therapy studies use plasmids of less than $10 \mathrm{~kb}$. To prolong gene expression, bacterial artificial chromosomes (BAC) have been used in non-dividing cultures. ${ }^{63}$ A BAC-plasmid vector encoding $185 \mathrm{~kb}$ DNA insert of human beta globin gene and EBV orip and EBNA-1 transactivator showed persistent gene expression indicating the usefulness of plasmid size. ${ }^{64} \mathrm{~A}$ episomal BAC vector encoding entire genomic human low-density lipoprotein receptor (LDLR) and LacZ showed high transfection and persistent gene expression of LDLR and LacZ. ${ }^{65}$ 
Bacterial components in the plasmid vector are known to elicit immunostimulation. Therefore, several research groups are investigating the possible use of minicircle plasmids, which do not have bacterial origin of replication or antibiotic resistance marker. These are generated in E.coli by site-specific recombination. Following injection of minicircle plasmid encoding $\mathrm{VEGF}_{165}$ into Balb/c mouse heart and skeletal muscle, VEGF $_{165}$ gene expression levels were similar or higher to that observed with conventional plasmid vectors encoding $\mathrm{VEGF}_{165}{ }^{66}$ In another study, minicircle mediated delivery of interferon- $\gamma$ (IFN- $\gamma$ ) was more efficient in inducing antiproliferative and antitumor effects in human nasopharyngeal cancer cell lines than conventional vectors due to its sustained expression of IFN- $\gamma$ levels. ${ }^{67}$ This suggests that minicircle plasmids may be a viable alternative to conventional plasmids vectors.

\subsubsection{Site-Specific Gene Expression}

Targeting of gene medicines to specific cells is often required to prevent toxicity to healthy cells and to decrease the required dose. Differential gene expression among cell types is possible because different genes are driven by different sets of promoters and enhancer sequences, and each of these regulatory binding sequences contain binding sites for multiple transcription factors. Changing the set of transcription factors will lead to the activation of a different set of genes, leading to a change in the cell's protein expression profile. Selective expression of transgenes in specific cells or tissues can be achieved by constructing DNA expression cassettes that contain gene regulatory regions that are recognized by transcription factors, specially present in or selectively expressed by the target cell population. This targeting is based on tissue specificity where transcription is directed specifically among healthy tissues or tumor specific by using elements which are active in tumor cells due to aberrant gene expression or tumor biology.

There are various well characterized regulatory elements controlling cell type specific expression, with target tissues including pancreas, breasts, bone, brain, kidney, bladder, lung, and liver. Tissue specific promoters display a natural activity in normal tissues without discriminating diseased cells from healthy ones. Therefore, for toxic protein expression the use of tissue specific promoters is limited to dispensable tissues such as melanocytes, prostate, breasts, endocrine, and exocrine tissues. ${ }^{12,68,69}$ Combining tissue specific promoters with additional targeting moieties can further increase their utility. For example, combination of tumor and tissue specific promoters may enable targeting specific cells/malignancies within nondispensable tissues. Cellular regulatory elements often have low activity, which can be addressed by inclusion of a strong promoter element from viral or cellular origin. For example, Pujal and colleagues have shown the specificity of the keratin 7 promoter which is expressed in pancreatic ductal cells predominantly than acinar cells which depends on the krt 7-234 bp sequence. This sequence is included in a plasmid or viral vector exhibits the same specificity as krt7 promoter and may help in targeting pancreatic ductal adenocarcinoma cells in vitro and in vivo. ${ }^{70}$ Targeting expression to a particular organ, tissue and cells greatly reduces the antibody response to a foreign protein and results in sustained expression. ${ }^{71}$ 
Most composite promoters contain one or two enhancer elements fused to a heterologous promoter sequence. This concept has been used to create promoters with combinations of regulatory sequences. Li et al., randomly assembled muscle-specific elements from four different muscle-specific promoters and then screened these novel promoters for activity. ${ }^{72}$ One sequence showed 8 -times the activity of natural muscle promoters. However, this was done in vitro. It is more challenging to develop a promoter with greater expression in vivo.

\subsubsection{Pulsatile Gene Expression}

Gene switches are introduced into expression systems to make use of circadian rhythm by extraneously administering compounds to control the production of therapeutic proteins by turning on or off the transcription of an administered gene. Gene switches are incorporated into the vector for gene regulation. This feedback control may prevent overexpression and possible deleterious protein production. In this system the target gene is inactive until the administration of an exogenous compound or ligand. ${ }^{73,74}$ Progesterone antagonists, tetracycline, ecdysone and rapamycin are used as inducing agents to turn on gene regulation of the expression vector.

\subsection{Viral Vectors}

Recombinant viruses are unique in being naturally evolved vehicles that efficiently transfer their genes into host cells. Viral vectors are composed of either a RNA or DNA core, or process different genomic structure and host ranges. However, they often have risks such as toxicity, immunogenicity, and/or potential for viral recombination. Novel developments of viral vectors mainly aim at reduction of immunogenicity and improved vector production. Several kinds of viruses including retrovirus, adenovirus (Adv), adeno-associated virus (AAV), herpes simplex virus (HSV), have been manipulated for gene transfer and are commonly used for modifying islet cells to improve their survival and enhance their metabolic activity. These viral vectors have their own unique advantages and disadvantages as discussed below.

\subsubsection{Retroviral Vectors}

Retroviruses are enveloped single stranded RNA viruses. Retroviruses have a genome of about 7-10kb, composed of gag, pol, and env genes flanked by elements called long terminal repeats (LTRs). These are essential for integration into the host genome and signify the beginning and end of the viral genome. The LTRs control the expression of viral genes, and hence act as enhancer- promoter. The final element of the genome, the packaging signal $(\psi)$, helps in differentiating the viral RNA from the host RNA. ${ }^{75}$ Gag proteins are major components of the viral capsid. Pol proteins are involved in the synthesis of viral DNA and integration into the host DNA, whereas env proteins play a role in association and entry of viral particles into the host cell. 
The viral genome can be manipulated and viral genes can be replaced by inserting transgenes. The transgenes can be controlled by the LTRs or alternate enhancer-promoter sequences can be engineered within the transgene. The chimeric genome is then introduced into packaging cell lines, which produce all the viral genes but these have been separated from the LTRs and the packaging sequence. So, the chimeric viral genomes are assembled to produce the retroviral vector. The culture medium containing the packaging cells which produce the retroviral vector is used directly to infect target cells for gene transfer.

Retroviral vectors have several advantages including stable transduction of dividing cells, less immunogenic and persistent transgene expression. However, there are several disadvantages of these vectors. These include random insertion into the host genome, limited DNA insertion capacity ( $8 \mathrm{~kb})$, low titers, and inactivation by complement systems, and inability to transduce non-dividing cells.

LIM-homeobox transcription factor islet-1 (Ls11) is a transcription factor derived from heart during embryogenesis that has the ability to self-replicate was delivered to the endothelial cells and mesenchymal stem cells (MSCs) using a retroviral vector resulting in promotion of angiogenic and vasculogenic properties. Lsl1 expressing endothelial cells also promoted vascularization in vivo in $\mathrm{C} 57 \mathrm{BL} / 6 \mathrm{~J}$ mice. $^{76}$

\subsubsection{Lentiviral Vectors}

Although lentiviral vectors belong to the retroviral family, they have the ability to infect both dividing and non-dividing cells. ${ }^{77}$ Human immunodeficiency virus (HIV) is the best known lentivirus. ${ }^{78}$ HIV lentiviral vector is very efficient as it has the ability to infect and express genes in human helper T cells and macrophages. Apart from the genes gag, pol and env, the HIV has six accessory proteins tat, rev, vpr, vpu, nef, and vif. These proteins regulate the synthesis and processing of viral RNA and other replicative functions. The accessory proteins can also be removed without affecting the production efficiency of the virus. The env gene from HIV-based vectors allows the infection of cells which express protein $\mathrm{CD}^{+}$, so in these vectors this is substituted with other env gene from other RNA viruses which have a broader infection spectrum.

The significance of lentiviral vectors lies in the fact that they can efficiently transduce non-dividing cells or terminally differentiated cells such as neurons, macrophages, hematopoietic stem cells, muscle and liver cells and other cell types for which gene therapy methods could not be used. These vectors, when injected into the rodent brain, liver, muscle, or pancreatic islet cells, show a sustained gene expression for over six months. ${ }^{79}$ These vectors do not show any immune response and no potent antibody response and thus can be ideal for in vivo gene delivery.

Magnetic nanoparticles have been used for the delivery of lentiviral vectors to endothelial cells. This method provides the direct targeting of the lentiviral vectors to endothelial cells even in perfused blood vessels apart from increasing the transduction 
efficiency. ${ }^{80}$ However, one problem with lentiviral vector is random integration into genomic DNA. This integration although desirable can be problematic as it can cause differential gene expression in the cells and most importantly insertational mutagenesis resulting in malignant transformation. To alleviate this effect non-integrating lentiviral vectors are developed by point mutations into the chromosome binding site, the viral DNA binding site of the viral integrase. ${ }^{81}$

There are reports in the literature where they have used lentiviral vectors encoding immunosuppressive proteins to protect the islets from allogenic rejection. ${ }^{82}$ They have showed that genetic engineering of beta-cells using gp19K-and RID alpha/betaexpressing lentiviral vectors can be a viable alternative to eliminate or reduce treatment with the potent immunosuppressive agents necessary at present for prolonged engraftment with transplanted islets.

\subsubsection{Adenoviral Vectors}

Adenoviruses are non-enveloped double- stranded DNA viruses, and can infect both dividing and non-dividing cells. Natural adenoviruses cause benign respiratory tract infection in humans. Their genome contains many genes and they do not integrate into the host DNA. Replication deficient adenoviruses can be generated by removing the E-1 gene necessary for viral replication and replacing it with the gene of interest (for example, hepatocyte growth factor) and a promoter sequence. ${ }^{83}$ These recombinant viruses are replicated in cells that express the product of the E-1 gene and generated in high concentrations. ${ }^{84}$

Cells infected with recombinant adenoviruses can express the therapeutic gene, but they cannot replicate as the genes needed for replication are absent. These vectors efficiently transduce cells and gene expression lasts for about 5-10 days. Therefore, Adv vectors are suitable for transient gene expression unlike retroviral vectors, which show long term expression. Adv vectors show extended duration of expression when given to nude mice or with an immunosuppressant indicating that the immune system may be responsible for the short duration of expression. ${ }^{85}$

The immune reaction elicits both cell killing 'cellular response' and antibody producing 'humoral response.' Cellular response results in the killing of infected cells with T-lymphocytes, whereas the humoral response results in the production of antibodies to Adv proteins, resulting in the diminished transgene expression following subsequent infections. ${ }^{85,86}$ Moreover, most human beings are likely to have antibodies to Adv from previous infections as it is a commonly found virus infecting the human population. To address this problem, second generation of Adv vectors were produced where the other genes of the Adv genome are deleted like the E-3 gene required for eliciting immune response. However, their expression was not longer than 20-40 days. ${ }^{87}$

Gutless Adv vectors are also generated in which all Adv genes are deleted. The viral DNA contains the start and end of the viral genome along with the viral packaging 
sequence. They showed expression for about 84 days. ${ }^{88}$ However, the production of these gutless Adv vectors is somewhat difficult. Furthermore, they still have immunological problems, which need to be overcome for their in vivo applications. One way of getting around this problem is to develop strategies to target the viral particles to the required cells, tissues, or organs. Targeting of vectors can ideally lead to less immunological responses and fewer amounts of viral particles are needed to get the desired therapeutic effect. However, Adv vectors transduce cells more efficiently only in the presence of coxsackievirus and adenovirus receptors (CAR). ${ }^{89,90}$ Immune response from Adv vectors can further be attenuated by deleting E4 or a part of the E4 region.

To target Adv vector to a specific cell or tissue usually Adv capsid, such as its fiber, protein IX (pIX), or hexon is altered. Of these, fiber proteins are the most studied. These proteins consist of a tail, shaft, and knob. ${ }^{91}$ The C-terminal knob is responsible for binding with CAR receptors. ${ }^{78,92}$ Targeting was achieved by incorporating an RGD peptide or a stretch of lysine residues (KKKKKKK [K7] peptide), targeting the $\alpha \mathrm{V}$ integrins, or heparin sulfate proteoglycans into the $\mathrm{HI}$ loop or C-terminal region of the fiber knob. ${ }^{93-95}$ pIX can also be altered to achieve targeted delivery using an Adv vector. ${ }^{96}$ pIX is a structural protein that sustains the structural integrity of the viral particles. Kurachi et al., reported the enhanced transduction efficiency of Adv vectors containing an RGD peptide in the c-terminus of pIX with $\alpha$-helical spacer. ${ }^{91}$ Also modification of hexon containing RGD peptides (DCRGDCF) at HVR 5 region in the Ad vector can render them infective via av- integrin receptor without any affinity to the CAR receptor. ${ }^{97}$ Therefore, modifications in the Adv capsid at pIX or hexon containing heterologous peptides render them more selective as compared to that at fiber in the capsid.

Biermann et al., have also shown that the modification of high-capacity Adv vectors by incorporating either 6X-His epitope or RGD peptide into the HI loop of the fiber knob rendered the vectors more effective and allowed efficient targeting towards different cell types. ${ }^{98}$ Targeting can also be achieved by affinity immobilization of Adv particles to the surfaces of biodegradable nanoparticles resulting in improved transduction through uncoupling cellular uptake from CAR receptor. ${ }^{99}$ The Adv nanoparticles containing inducible nitric oxide synthase (iNOS) inhibited growth of culture smooth muscle cells more compared to Adv encoding reporter gene GFP without any therapeutic activity $\left(\mathrm{Ad}^{\mathrm{GFP}}\right)$ or Adv without any gene (Ad free vector).

Islets are non-replicating cells which secrete insulin following an increase in blood glucose levels in the body. When there is death of islets due to genetic or immune mediated defects, one of the major alternatives is transplantation of islets. However, due to inadequate vascularization and subsequent immune attacks, islet transplantation did not achieve the success it was meant to. We have shown that human vascular endothelial growth factor (hVEGF) expression promotes new blood vessel formation and improves the outcome of islet transplantation. ${ }^{100}$ To enhance hVEGF gene expression, we transduced human islets with bipartite Adv vectors encoding hVEGF and human interleukin-1 receptor antagonist (hIL-1Ra) to promote revascularizaton and protect the human islets from apoptosis. ${ }^{101}$ Bipartite vector results in simplifying the amplification and purification of Adv vectors but also minimizes the Adv backbone for transduction, 
thereby lessening the immunogenic effects of the vector. There was dose dependent increase in expression of hVEGF and hIL-1Ra from islets transduced with Adv-hVEGFhIL-1Ra. We confirmed whether the Adv vector is causing any detrimental effects to the islets by measuring the stimulation index of islets following transduction by Adv vector. Islets transduced by Adv vectors were as functional as untransduced islets. There was a decrease in caspase-3 levels in Adv transduced islets compared to untransduced islets when they were incubated with inflammatory cytokines. The expression was several times higher than the bicistronic plasmid vector phVEGF-hIL-1Ra. Following transplantation of Adv-hVEGF-hIL-1Ra transduced islets in non-obese diabetic severe combined immunodefecient (NOD-SCID) mice, there was a decrease in blood glucose levels and increase in insulin and c-peptide levels.

Over-expression of hVEGF can be detrimental to islets and surrounding tissues and can lead to development of tumors. ${ }^{102,103}$ On the other hand hepatocyte growth factor (HGF) is a potent mitogen of human islets and is known to promote $\beta$-cell proliferation and is also anti-apoptotic. ${ }^{104} \mathrm{We}$ therefore constructed a bipartite Adv vector encoding hHGF and hIL-1Ra (Adv-hHGF-hIL-1Ra) driven under a separate CMV promoter. ${ }^{101}$ The vector showed increase in hHGF and hIL-1Ra genes with increase in multiplicity of infection (MOI) and duration. It did not affect the function of islets and showed decreased caspase-3 levels compared to the untransduced islets. Transduction of islets with Adv-hHGF-hIL-1Ra enhanced the level of Bcl-2 protein and inhibited the levels of Bax protein demonstrating the protective effect of hHGF and hIL-1Ra co-expression. There was also decrease in blood glucose levels and increase in insulin and c-peptide levels in NOD-SCID mice transplanted with Adv-hHGF-hIL-1Ra transduced islets. Immunohistochemcial staining of islet bearing kidney sections revealed stronger positive staining for human insulin, hHGF and hvWF suggesting more efficient blood vessel formation in Adv-hHGF-hIL-1Ra transduced islets. This shows that Adv vectors can be utilized for efficient and high expression of transgenes with less immunogenic effects compared to non-viral vectors. However, these vectors can still elicit immune responses more so in vivo setting. Therefore, by further removing the E4 or part of E4 genome of the Adv vectors and by selectively targeting the Adv vector to a specific organ or tissue by attaching a ligand (Gal-PEG) to it can further enhance the prospects of Adv vectors in gene therapy.

\subsubsection{Adeno-Associated Viral Vector}

Adeno-associated virus (AAV) is a simple non-pathogenic single-stranded DNA virus and is a member of parvoviridae family. AAV is composed of two open reading frames (ORF), rep and cap, and two inverted terminal repeats (ITRs) that define the start and end of the viral genome and packaging sequence, ${ }^{105}$ whereas the cap gene encodes viral capsid (coat) proteins and rep gene is for replication and integration. AAV requires additional genes to replicate, which are usually provided by an Adv or a herpes simplex virus (HSV). The AAV vector is produced by replacing the rep and cap genes with the transgene. Only one out of 100-1000 viral particles is infectious. Apart from the production of AAV vector being laborious, these vectors also have the drawback of 
limited packaging capacity for the transgene $(4.7 \mathrm{~kb})$. Furthermore, there are no packaging cells which can express all the proteins of the virus. Since rAAVs are deleted of viral genes, these vectors are less immunogenic. However, specific circulating bodies to rAAVs have been detected limiting their potential administration. ${ }^{106}$

AAV vectors are the most promising vectors for gene therapy in improving islet transplantation outcomes mostly due to their low immunogenicity and high safety profile. ${ }^{107}$ AAV vectors are commonly used to deliver immunosuppressive genes to islets prior to transplantation resulting in improving the longevity and function of transplanted islets. ${ }^{108}$ Interleukin-10 (IL-10) gene was delivered to the islets using an AAV vector encoding IL-10 (AAV-IL-10) protected the islets from autoimmune recurrence and allogenic rejection. ${ }^{109}$ AAV-IL-10 mediated delivery of IL-10 mitigated the previously activated T-cells and protected the islets from immune rejection.

\subsubsection{Other Vectors}

HSV, which infects the cells of nervous system, is being developed as a vector. This virus contains 80 genes, of which one can be replaced to produce the vector. ${ }^{51}$ The use of recombinant baculoviruses, containing mammalian regulatory elements for efficient transient and stable transduction of different mammalian cell types is being explored. ${ }^{110}$ Alpha viruses are being used in the development of vaccines. ${ }^{11,112}$

\subsection{Gene Delivery Systems}

Gene delivery systems help in controlling the location of a gene within the body by regulating the biodistribution of a gene expression system. They aid in protecting the gene expression systems from premature degradation in extracellular milieu and allowing non-specific or cell-specific targeting of the expression system. Some delivery systems are designed for specific targeting to a receptor or aid in intracellular trafficking of the gene expression system. Some of the common gene delivery systems used are discussed below.

\subsubsection{Liposomes}

Plasmids are generally complexed with cationic liposomes to protection them from in vivo degradation and to enhance intracellular delivery. Cationic lipids are composed of a hydrophobic lipid anchor group, linker group and a positively charged head group. Cationic lipids present in the liposome interact electrostatically with negatively charged phosphate backbone of DNA, thereby condensing the DNA into a more compact structure. These are most commonly used synthetic carriers for delivery of oligonucleotides, siRNAs, and plasmid DNA. The degree of transfection by cationic liposomes mainly depends on the extent of DNA condensation, cellular uptake by 
interaction with biological surfaces and membrane fusion via transient membrane destabilization for cytoplasmic delivery avoiding lysosomal degradation.

Although $\mathrm{pH}$ sensitive liposomes can be used to encapsulate DNA, low entrapment efficiency and high serum sensitivity limits their usage. This scenario changed with the introduction of the first cationic lipid 2, 3-dioleyloxypropyl-1-trimethyl ammonium bromide (DOTMA). ${ }^{113}$ Most of the research is being done to increase the transfection efficiency through the modification of the functional headgroups. Also, enhancing the transgene expression has become the focus of many studies. New cationic lipids were developed by modifying the headgroups of DOTMA resulting in increased transfection efficiency.(( \pm )-N-(2-Hydroxyethyl)-N,N-dimethyl-2,3-bis(tetradecyloxy)-1propanaminium bromide (DMRIE), which was synthesized by modifying DOTMA showed increase in transfection efficiency and transgene expression. ( $3 \beta\left(\mathrm{N}_{-}\left(\mathrm{N}^{\prime}, \mathrm{N}^{\prime}\right.\right.$ Bimethylaminoethane) carbamoyl) cholesterol (DC-Chol) was the first cholesterol based cationic lipid. ${ }^{114}$ Its structure was further modified by conjugating to polyamino groups resulting in more positive charges. ${ }^{114}$ Spermine and spermidine were conjugated to cholesterol to reduce cytotoxicity and increase the delivery of antisense oligonucloetides. ${ }^{61}$ Changing spermine or spermidine into a secondary amine showed better transfection efficiency both in vitro and in vivo. ${ }^{115}$ Some of the cationic lipids such as DC-Chol, DMRIE, and GL-67 have been used in early clinical trials, but the results were not encouraging. Hetreocyclic cationic lipids have also been studied for gene delivery. Some of these compounds showed better transfection abilities and lower cytotoxicity compared to cationic lipids with linear primary amines or polyamines as head groups. ${ }^{114}$

Pyridinium-based cationic lipids have been shown to have similar or higher transfection efficiency compared to commercially available cationic lipid formulations. Pyridinuim lipids displayed higher transduction efficiency in cells which are not easily transfected by other cationic lipids like Lipofectin, and transfect relatively a large group of cells. ${ }^{116}$ Zhu et al., have synthesized a series of pyridinium lipids containing a heterocyclic ring and a nitrogen atom and prepared liposomes with co-lipid L- $\alpha$ dioleoylphosphatidylethanolamine (DOPE) and cholesterol (Chol) by sonication. ${ }^{117}$ Pyridinium lipids with amide linker showed significantly higher transfection efficiency compared to their ester counterparts. Liposomes prepared at a 1:1 molar ratio of pyridinium lipid and a colipid showed higher transfection efficiency when either DOPE or cholesterol was used as a colipid to prepare liposomes. The pyridinium lipids with a trans-configuration of the double bond in the fatty acid chain showed higher transfection efficiency than its counterparts with cis-configuration at the same fatty acid chain length. In the presence of serum, C16:0 and lipofectamine significantly decreased their transfection efficiencies, which were completely lost at serum concentration of $30 \%$ and higher, while C16:1 trans-isomer still had high transfection efficiency under these conditions.

The liposomal vector interacts with extracellular components in serum after in vivo administration. This often causes failure of the liposomal vector to reach the target cells. To have a targeted liposomal vector system, a ligand is required to bind to the receptor of 
the target cells. Nasopharyngeal epidermal carcinoma cells which express excess of folate receptors were targeted by liposome-entrapped polycation-condensed DNA (LPDII) anionic liposome encapsulating polylysine condensed DNA tagged with folate. ${ }^{118}$ $\mathrm{Lys}_{2} \mathrm{Gal}_{3}$, an aminogalactoside was used to target hepatoma in vitro ${ }^{119}$ which is a cationic formulation consisting of lipopolymaine, DOPE (helper lipid) and a galactolipid (DPPE$\mathrm{Lys}_{2} \mathrm{Gal}_{3}$ ). Lipososmal vectors have been used to improve the islet transplantation process. Hypoxia is one of the major factors resulting in primary non-function of transplanted islets. VEGF gene was delivered to the islets using a cationic lipid which increased the transfection efficiency and resulted in better glycemic control of the transfected islets. ${ }^{120}$ Tacrolimus was sufficiently encapsulated in liposomes and delivered to the islets resulting in improved immunosuppressive effect. ${ }^{121}$ However, toxicity at higher doses is a major concern.

\subsubsection{Cationic Peptides}

Major difficulties in non-viral delivery are the ability of the vectors to condense the DNA, targeting to specific cells, disrupt the endosomal membrane and deliver it to the nucleus. Peptide based vectors can be successfully employed to overcome these barriers. Cationic peptides condense DNA by interacting with its negatively charged phosphate backbone.

Poly (L-Lysine) (PLL) is one of the first cationic peptides to deliver genes. However, increase in length of PLL leads to cytotoxicity. It shows very less transfection efficiency and needs another fusogenic peptide to facilitate plasmid release into the cytoplasm. Since PLL cannot escape from the endosome without addition of endoosmolytic agents such as chloroquine, Midoux and Monsingy have constructed a histidine-substituted polylysine, since histidine protonates at acidic $\mathrm{pH}$ in the endosome. Cationic peptides can be linked to cell specific ligands and bound to plasmids through electrostatic interaction. These complexes can interact specifically with target cell receptors leading to internalization of the complex into the cells.

Synthetic peptides which are conjugated to lipids usually show better binding ability to DNA. For example, inclusion of dialkyl or diacyl chains in the cationic peptides improves their ability to bind to DNA and reduces aggregation of complexes in ionic media. ${ }^{9,31,122}$ Synthetic peptides derived from the N-terminus of influenza virus haemagglutinin and the rhinovirus VP-1 protein, ${ }^{123}$ or artificial amphipathic peptides were used. ${ }^{31}$ These peptides may have specificity for endosomal $\mathrm{pH}$ due to acidic residues (glutamic and aspartic acids) aligning on one side of an amphipathic helix. At neutral $\mathrm{pH}$, the negatively charged carboxylic groups destabilize the alpha-helical structure resulting in the multimerization of peptides and/or membrane interaction. The $\mathrm{pH}$ specificity can be enhanced by introducing additional glutamic acids into the peptide sequence. ${ }^{124}$ The enhancement of gene expression is strongly dependent on the peptide sequence. The activity of cationic lipid/DNA complexes has been enhanced by mixing with Sendai virus envelopes. This approach and related systems utilizing neutral lipids 
and liposome-like reconstituted envelopes (virosomes) based on Sendai virus or influenza viruses have been found effective for gene delivery. ${ }^{125}$

Amphipathic cationic peptides such as, gramicidin S, is incorporated into a DOPE lipid/DNA composition to facilitate gene delivery. Incorporation of gramicidin S and DNA into asialofetuin-labeled liposomes was used for receptor-mediated gene delivery into primary hepatocytes. ${ }^{126}$ The influence of influenza virus-based peptides on cationic lipid-based transfection was studied. ${ }^{127}$ Wagner et al., showed that the use of positively charged lipospermine/DNA complexes resulted in 3 to 30 -fold enhancement in gene expression by association with the peptides. ${ }^{128}$ Kamata et al., also showed that influenzaderived peptides can increase the level of gene expression of a lipofectin formulation by up to five-fold. ${ }^{129}$ Thus, escape from endocytic vesicles does not seem to be a major barrier for optimized, positively charged DNA/lipospermine or lipofectin complexes. However, for less positively charged lipospermine complexes gene transfer efficiency was found to be increased by a factor of 50 to 1000 by synthetic peptides INF6 (influenza virus derived sequence) and INF10 (artificial sequence). ${ }^{128}$ Wilke et al., generated novel DNA complexes containing a palmitoyl-modified DNA-binding peptide showing enhanced transfection activity. Gene transfer was found to be restricted to mitotic cells. ${ }^{130}$

Membrane-modifying peptides are able to enhance both lipofection and polyfection. Transfection efficiency of cationic peptide-based systems are strongly dependent on the presence of endosomolytic peptides or related agents (like glycerol, viral particles) which enhance cytoplasmic delivery. ${ }^{123}$ Transfection efficiencies can be improved up to more than 1000 -fold by endosomolytic compounds. ${ }^{128}$

It is important to determine (i) whether membrane-modulating peptides and DNA carriers can influence other intracellular steps beside endosomal escape or cell membrane fusion, such as the transport of the DNA into the nucleus of the cell; and (ii) whether the results obtained with membrane-active peptides in cell culture can also be exploited for in vivo gene transfer. ${ }^{125}$ Cationic peptides can be used for modifying the surface of islets to prevent inflammation mediated loss of islet function following islet transplantation. Teramura et al., modified the surface of islets was modified with a poly (ethylene glycol)-phospholipid conjugate bearing a biotin group (biotin-PEG-lipids, PEG MW:5000). ${ }^{131}$ This resulted in improved islet function as surface modification mitigated the blood mediated inflammation.

\subsubsection{Cationic Polymers and Lipopolymers}

Polyethyleneimine (PEI), polypropylenimine, and polyamidoamine dendrimers have been employed for gene delivery. PEI $^{117}$ is a branched cationic polymer widely used in the gene delivery. It condenses the plasmids into colloidal particles that effectively transfect genes into a variety of cells in vitro. ${ }^{132}$ PEI contains several secondary amines which get protonated in the acidic environment of the endosome. This leads to endosomal swelling and subsequent membrane disruption leading to the release of the vector into the cytosol. However, high molecular weight PEI ( $>25 \mathrm{kDa})$ show cytotoxicity and 
$\mathrm{PEI} /$ plasmid vector complexes under aggregation on storage, resulting in less gene expression. Chemical modification of low molecular weight PEIs may improve transfection and reduce cytotoxicity.

Mahato and associates synthesized a water soluble lipopolymer by conjugating cholesteryl chloroformate to branched PEI of $1800 \mathrm{Da}$ through primary and secondary amines. ${ }^{133}$ This polymer was non-toxic to CT-26 colon carcinoma cells and did not cause any aggregation compared to PEI/DNA complexes. Cholesterol will promote both micellar formation and hydrophobic interaction with plasmid and cellular membrane while PEI will condense DNA via electrostatic interaction. However, transfection of islets with lipofectamine showed less transfection efficiency as islets are approximately a cluster of 1000 non-dividing cells an new strategies are needed for improving the transfection efficiency. ${ }^{134}$

\subsubsection{Hybrid Vectors}

Both viral and non-viral vectors have their own advantages and disadvantages. There is much work going in developing new ways of better utilizing these vectors for gene expression and delivery. One such approach is to overcome the limitations of individual vectors by combining them. These vectors are called hybrid vectors. For example, the adenovirus hexon protein enhances the nuclear translocation and increases the transgene expression of PEI/pDNA complexes. ${ }^{135}$ PEG has been widely used for conjugating with adenoviral vector to prolong its circulation half-life, enhance transgene expression and prevent immune activation. ${ }^{136}$

Cationic lipids and polymers may help in improving the transduction efficiencies of viral vectors. These are particularly useful in cells which do not have the specific viral receptors. The cationic nature of these molecules may promote binding to the negatively charged viral capsid altering the cell surface and allow internalization of viral particles. This can also elicit very less immunostimulation. For example, cationic liposomes promote delivery of Adv vectors into target cells which lack the CAR receptors and $\alpha v-$ integrin receptors improving transgene expression. ${ }^{137}$ Diamond and his colleagues have showed that delivery of Adv vectors associated with dexamethasone-spermine (DS) conjugate to the lung enhanced targeting to the conducting airway epithelium and reduced immune response. ${ }^{138}$ Further, formulation of Adv encoding LacZ with DS/DOPE allowed re-administration of the adv vector, with little loss of transgene expression.

Adv vector can also be coated with polymers bearing side chains containing positively charged quaternary amines and carbonyl thiazolidine-2 thione groups to prevent binding of Adv vector to plasma protein and consequently prolong blood circulation half-life and more deposition of the vector at diseased sites. ${ }^{139}$ 


\subsubsection{Receptor-Mediated Gene Transfer}

Targeting ligands have been incorporated into DNA complexes for site or cell specific gene delivery. ${ }^{125}$ By attaching the DNA to a domain that can bind a cell surface receptor, such as the asialoglycoprotein receptor, transferrin receptor, the efficient cellular process of receptor-mediated endocytosis, can be utilized. Conjugates with polylysine, protamines, histones, PEI, cationic lipids, and other polycations have been generated and tested for receptor-mediated endocytosis. Hong and his colleagues used a hydroxycamptothecin (HCPT) loaded polyethylene glycol (PEG) niosomes attached with transferrin to the terminal group of PEG. This system showed high antitumor activity compared to non-transferrin bound niosomes. ${ }^{117}$ To enhance the uptake and specificity, $\mathrm{Wu}$ and $\mathrm{Wu}$ generated a polylysine-based, asialoglycoprotein receptor-specific gene delivery system by incorporation of asialoorosomucoid-polylysine conjugates into DNA complexes. ${ }^{140,141}$ Complexes could be efficiently delivered into endosomes or other internal vesicles of cells, but they were still separated from the cytoplasm by a membrane. This accumulation of complexes in internal vesicles strongly reduced the efficiency of gene transfer. Receptor-mediated delivery into a hepatocyte cell line resulted in uptake of DNA into practically all cells, but only a few cells expressed the delivered gene. ${ }^{142}$

\subsection{Concluding Remarks}

Although significant progress has been made to employ gene therapy in the clinic to treat various severe and debilitating diseases, there is still no FDA approved products in the market. This can be attributed to the lack of proper gene expression and delivery systems. While viral vectors efficiently transduce cells and have shown promise in their clinical trials, there are still some safety concerns, especially immunostimulation and integration within the host. Although non-viral vectors are relatively safe, transgene levels are very low and transient and thus many vectors have failed to perform well in the clinical trials. Therefore, efforts are being made to generate hybrid systems by combining the beneficial effects of non-viral and viral vectors. This has led to the significant enhancement in the gene expression, with minimal toxicity and immunogenicity. Further, the use of cellular promoters offers targeting of the vector to specific target tissues rendering the vector safe and efficacious. There is a definite need to develop targeting strategies for non-viral vectors which can compensate for the inefficient gene transfer.

Islet transplantation usually results in decrease of hypoglycemic episodes which is a major drawback for conventional insulin therapy. Gene therapy has resulted in mitigating the hypoglycemic episodes and improved the graft function and showed higher levels of insulin and c-peptide levels in the majority of patients. However, this can last only for a few years after which patients have to resort to insulin therapy. Gene therapy can be implemented in this case to prevent the toxic effects of immunosuppressive agents and control the inflammation and immune reactions following islet transplantation. With recent improvements in gene delivery and increased understanding of the mechanisms 
underlying graft failure, gene therapy for islet transplantation has the potential to move closer to the clinic as a treatment for patients with Type I diabetes. 


\section{CHAPTER 2. BIPARTITE VECTOR ENCODING hVEGF AND hIL-1Ra FOR EX VIVO TRANSDUCTION INTO HUMAN ISLETS}

\subsection{Introduction}

Type I diabetes is an autoimmune disease that results in the destruction of insulin producing $\beta$-cells of the pancreatic islets. Significant progress has been made in the field of islet transplantation where islets are transplanted via the portal veins of Type I diabetic patients. However, a large number of islet grafts do not secrete insulin, as they fail to revascularize upon transplantation and undergo apoptotic cell death. ${ }^{143}$

Islets have an intricate microvasculature, which is disrupted during the isolation and purification process. Revascularization to the transplanted islets is known to improve the delivery of nutrients and oxygen to the inner core of islets leading to their survival. ${ }^{100,133,144,145}$ Transduction of islets with an adenoviral (Adv) vector encoding hVEGF or hepatocyte growth factor has been shown to promote the formation of new blood vessels, leading to improved islet survival. ${ }^{100,133,145,146}$ Apart from revascularization, islet grafts also need to be protected from apoptosis induced by inflammatory cytokines such as interleukin-1 $\beta$ (IL-1 $\beta$ ), tumor necrosis factor-alpha (TNF- $\alpha)$ and interferon- $\gamma(\mathrm{IFN}-\gamma)$ released from resident macrophages and infiltrating immune cells at the transplantation site. ${ }^{147}$ Immunosuppressive medications are used simultaneously to protect transplanted islets, but reports of severe side effects induced by immunosuppressive drugs have prompted attention on developing ways to reduce their toxicities. ${ }^{148}$ Among various cytokines, IL-1 $\beta$ plays a key role in apoptotic cell death. Inhibition of IL-1 $\beta$ receptor binding using interleukin-1 receptor antagonist (IL-1Ra) resulted in improved islet survival and function. ${ }^{149-151}$

Considering the favorable uses of hIL-1Ra and hVEGF, we recently constructed a plasmid vector encoding these two genes (phVEGFhIL-1Ra) by cloning hIL-1Ra under the cytomegalovirus (CMV) promoter and hVEGF under the elongation factor-1R(EF$1 \mathrm{R})$ promoter in pBudCE4.1 vector, and demonstrated dose and time dependent expression of hVEGF and hIL-1Ra at after transfection. ${ }^{18}$ Since transfection efficiency of this bipartite plasmid into human islets was very low and the better known transduction efficiency of Adv vectors, in this study, we constructed a bipartite Adv vector encoding hVEGF and hIL-1Ra driven by separate CMV promoters. The use of bipartite vector encoding these genes not only simplifies the amplification and purification process of $\mathrm{Adv}$ vectors but also decreases the use of total Adv backbone for transduction, which is expected to minimize immunogenic and toxic effect of Adv vectors. We determined transduction efficiency, islet function and caspase-3 release upon incubation with inflammatory cytokine cocktail of TNF- $\alpha$, IL- $1 \beta$ and IFN- $\gamma$. We then transplanted islets under the kidney capsules of NOD-SCID mice after transduction with Adv-hVEGF-hIL$1 \mathrm{Ra}$ and determined the blood glucose, insulin and c-peptide levels. Finally, the islet bearing kidney sections of these diabetic mice were stained for human insulin, hVEGF and von Willebrand factor (hvWF). 


\subsection{Materials and Methods}

\subsubsection{Materials}

Agarose, tris-borate-EDTA buffer, goat serum, glucose and haematoxylin solution were purchased from Sigma-Aldrich Co. (St. Louis, MO). Fetal bovine serum (FBS) and CMRL-1066 media were purchased from MediaTech Cellgros (Herndon, VA). Human VEGF and IL-1Ra ELISA kits and cytokines IL-1 $\beta$, TNF- $\alpha$, and IFN- $\gamma$ were purchased from R\&D Systems (Minneapolis, MN). Human Insulin ELISA kits were purchased from Alpco Diagnostics (Windham, NH), and RNA extraction and Caspase-Glo 3/7 assay kits from Promega (Madison, WI). SYBR Green real time PCR master mix and reverse transcription reagents were purchases from Applied Biosystems (Foster City, CA).

\subsubsection{Methods}

The methods used in this study are described below.

\subsubsection{Construction of Adv-hVEGF-hIL-1Ra}

A bipartite Adv vector encoding hVEGF and hIL-Ra genes was constructed with the help of OD 260, Inc. (Boise, ID). Briefly, hIL-1Ra cDNA along with rabbit $\beta$-globin polyA was cloned into $\mathrm{pE} 3.1$ shuttle plasmid, while hVEGF fragment along with rabbit $\beta$-globin polyA was inserted into $\mathrm{pE} 1.2$ shuttle plasmid. The $\mathrm{pE3}$.1-hIL-1Ra plasmid was further modified by removing the intron adjoining CMV promoter. These plasmids were transformed in super competent TOP 10 cells and amplified in terrific broth media. pE1.2-hVEGF and pE3.1-hIL-1Ra were digested to excise these expression cassettes for ligation with SfiI digested AdenoQuick 13.1 plasmid simultaneously using T4 DNA ligase overnight at $16^{\circ} \mathrm{C}$. The ligated products were packaged into phage lambda particles using MaxPlax packaging extract (Epicentre, Madison, WI). The packaging mixture was used to infect E.coli XL-1Blue (Stratagene, La Jolla, CA) and clones were selected on LB supplemented with $25 \mu \mathrm{g} / \mathrm{ml}$ kanamycin and $50 \mu \mathrm{g} / \mathrm{ml}$ ampicillin. The Pac I linearized cosmid was transfected into 293 cells to rescue the virus. Three viral plaques were amplified, and their genomic DNAs were extracted. The presence of hVEGF and hIL1Ra expression cassettes was confirmed by restriction analysis with Hind III and Not I. The clone containing the two genes was transduced to 293 cells, and the viral extract was further transduced into 293 cells, amplified and purified on two $\mathrm{CsCl}$ gradients. The bands harvested were dialyzed against GTS buffer $(25 \%$ glycerol, $25 \mathrm{mM} \mathrm{NaCl}, 20 \mathrm{mM}$ Tris- $\mathrm{HCl}, \mathrm{pH} 8$ ) and viral suspension was collected, filtered and stored at $-70^{\circ} \mathrm{C}$. Once the construction was confirmed for the insertion of genes hVEGF and hIL-1Ra, the virus was tested for expression of these genes in vitro and then in vivo. 


\subsubsection{Determination of Viral Titer}

The following methods were used to calculate the viral titer.

- OD 260-SDS Method: Adv-hVEGF-hIL-1Ra was purified using CsCl density gradient. The purified virus was diluted at different concentrations in the range of $0.1-1.0$ in $0.1 \%$ sodium dodecyl sulfate (SDS) buffer. The absorbance was recorded at $260 \mathrm{~nm}$. The viral titer was calculated based on the extinction coefficient of $1.1 \times 10^{12}$ virus particles (VP) per Abs260 unit in the presence of SDS buffer.

- Plaque Assay: Freshly confluent $6 \mathrm{~cm}$ dishes seeded with 293 cells were infected with various dilutions of Adv-hVEGF-hIL-Ra. Three days later, the cells were split into $10 \mathrm{~cm}$ dishes to boost virus plaque formation. The cells were split again into $15 \mathrm{~cm}$ dishes 10 days later. The formation of virus plaques was monitored up to 3 weeks after which the infection titer was calculated.

- Infectious Viral Titers: The infectious viral titer was also determined by using an Adeno-X rapid titer kit from Clontech. Different dilutions of Adv-hVEGFhIL-1Ra was taken and transduced to AD-293 cells, using untransduced cells as a control. After $48 \mathrm{~h}$ post-transduction, AD-293 cells were fixed and incubated with Anti-hexon antibody, followed by a secondary horse radish peroxidase (HRP)-conjugated antibody. Then the cells were stained with 3, 3' diaminobenzidine tetrahydrochloride (DAB) substrate and positively stained cells were counted under a microscope, and the titer was determined.

\subsubsection{Islet Culture and Transduction}

Human islets were received from one of the several Islet Cell Resource (ICR) Centers in culture media at $4^{\circ} \mathrm{C}$. On receiving, the islets were cultured in CMRL-1066 medium containing 10\% fetal bovine serum (FBS) and 1\% Penicillin Streptomycin. Islets were then transduced with Adv-hVEGF-hIL-1Ra, while non-transfected islets as well as the islets transfected with phVEGF-hIL-1Ra/Lipofectamine complexes $(3 / 1,+/-, 2.5 \mu \mathrm{g}$ DNA/1000 islets) were used as controls. To determine the optimal multiplicity of infection (MOI) of Adv vectors in terms of plaque forming units (pfu) per cell of an islet which is a cluster of 1000 cells, 1000 islets were incubated with Adv-hVEGF-hIL-1Ra in $300 \mu \mathrm{l}$ media in a 96 well plate for $12 \mathrm{~h}$, followed by washing and further culturing in $1 \mathrm{ml}$ of media in 24-well plates. Gene expression was determined at day 1 and 3 by quantifying secreted protein in the media by ELISA (R\&D Systems). Total protein of the islet cell extracts was also determined using the bicinchoninic acid (BCA) protein assay kit.

\subsubsection{Quantitative Real time PCR}

To determine hVEGF and hIL-1Ra gene expression 3 days after transduction with Adv-hVEGF-hIL-1Ra, the islet pellet was collected for isolation of total RNA using 
RNAeasy mini isolation kit from Promega. Two hundred and fifty nanograms (ng) of extracted RNA was converted into cDNA using MultiScribe reverse transcriptase and random hexamers (Applied Biosystems, Inc.) by incubation at $25^{\circ} \mathrm{C}$ for $10 \mathrm{~min}$, followed by reverse transcription at $48^{\circ} \mathrm{C}$ for $30 \mathrm{~min}$ and enzyme inactivation at $95^{\circ} \mathrm{C}$ for $5 \mathrm{~min}$. In all $3.85 \mu 1$ of cDNA was amplified by real time RT-PCR. To determine the level of hVEGF expression due to Adv-hVEGF-hIL-1Ra transduction, while excluding the endogenous expression, Adv vector specific reverse primer (5'-TAG CCA GAA GTC AGA TGC TCA AGG-3') was designed from the PolyA region specific to the vector. The forward primer (5'-TTT CTG CTG TCT TGG GTG CAT TGG-3') was designed from the hVEGF gene sequence. We also designed both primers from the gene: forward (5'-TTT CTG CTG TCT TGG GTG CAT TGG-3') and reverse (5'-AAG ATG TCC ACC AGG GTC TCG AAT-3') so that cumulative expression of hVEGF can be determined.

In case of hIL-1Ra both, forward (5'-ATC CAG CAA GAT GCA AGC CCT-3') and reverse (5'-TTC GTC AGG CAT ATT GGT GAG GCT-3') primers were designed from the gene sequence (NCBI Accession\#AK290898). To determine the level of hIL-1Ra expression excluding its endogenous expression, forward primer was designed from the Adv sequence (5'-AGC CTC ACC AAT ATG CCT GAC GAA-3') and reverse primer from the gene sequence (5'-TTG CCA TGC AAG AAT GGG AAC AGG-3') (NCBI Accession\#AB021221). The PCR conditions included denaturation at $95^{\circ} \mathrm{C}$ for $10 \mathrm{~min}$, followed by 40 cycles of amplification by sequential denaturation at $95^{\circ} \mathrm{C}$ for $15 \mathrm{sec}$ and primer annealing as well as strand extension for $1 \mathrm{~min}$.

To confirm the amplification specificity, the PCR products were subjected to melting curve analysis. hVEGF and hIL-1Ra gene expression was normalized to 18s rRNA as internal control. Threshold cycle number was compared between Adv-hVEGF-hIL-1Ra with the same parameters using non-transfected islets as controls.

\subsubsection{In Vitro Islet Function after Transduction}

The in vitro islet function after transduction with Adv-hVEGF-hIL-1Ra was determined by the static incubation method, using non-transduced islets as control. Transfection media was removed and the islets were sequentially incubated at $37^{\circ} \mathrm{C}$ for $1 \mathrm{~h}$ in the media containing $2.5 \mathrm{mM}$ (basal) and $22 \mathrm{mM}$ glucose (stimulated). After incubation, supernatants were collected and analyzed for insulin release by ELISA (Alpco Diagnostics). Insulin secretion was expressed as $\mu \mathrm{U} / \mathrm{ml}$ and the ratio of insulin levels at $22 \mathrm{mM}$ to $2.5 \mathrm{mM}$ glucose was used to calculate the stimulation index.

\subsubsection{Cytoprotective Effect of Adv-hVEGF-hIL-1Ra}

One thousand islets per well of 96 well plates were transduced with $1000 \mathrm{MOI}$ of Adv-hVEGF-hIL-1Ra, Adv-hVEGF or Adv-hIL-1Ra. At 12h post-transduction, islets were washed and incubated with either fresh media or a cocktail of IL-1 $\beta(10 \mathrm{ng} / \mathrm{ml})$, 
TNF- $\alpha(10 \mathrm{ng} / \mathrm{ml})$ and IFN- $\gamma(50 \mathrm{ng} / \mathrm{ml})$. At day 3 post transduction islets were collected from each well and lysed with lysis buffer to measure the caspase- 3 concentration using the Caspase-Glo 3/7 Assay kit (Madison, WI).

We also determined the cytoprotective effect of Adv-hVEGF-hIL-1Ra on islet viability by fluorescence microscopy of islets after transduction with Adv-hVEGF-hIL$1 \mathrm{Ra}(500,1000$ and $2000 \mathrm{MOI})$, Adv-hVEGF (1000 MOI), and Adv-hIL-1Ra (1000 MOI) for $12 \mathrm{~h}$, followed by incubation with the cytokine cocktail for 3 days. These islets were stained with propidium iodide $(500 \mathrm{nM})$ to identify dying or dead cells under fluorescence microscope (Olympus).

\subsubsection{Islet Transplantation Studies}

Animal experiments were performed as per the NIH (http:/grants1.nih.gov/grants/olaw/ references/phspol.htm) and institutional animal care and use guidelines using approved protocols. To establish the diabetic animal model, streptozotocin (STZ) $(40 \mathrm{mg} / \mathrm{kg})$ was administered to NOD-SCID mice by intraperitoneal injection for 5 consecutive days. Animals were considered to be diabetic after two consecutive blood glucose measurements $\geq 325 \mathrm{mg} / \mathrm{dl}$ using a One Touch Ultra Glucometer (Lifescan, Milipitas, CA). Before transplantation, human islets were transduced by Adv-hVEGF-hIL-1Ra or Adv-LacZ at the dose of $1000 \mathrm{MOI}$ for $12 \mathrm{~h}$ and washed with PBS. About 1500 transduced or non-treated islets were transplanted under the left kidney capsules of diabetic mice. The nonfasting glucose levels were measured up to 20 days post-transplantation. The mice were then anesthetized to collect blood to measure serum insulin by ELISA and C-peptide by radioimmunoassay (Diagnostic Products Corp.). The graft-bearing kidneys were removed to confirm the function of islet grafts by the return to blood glucose levels to $\geq 325 \mathrm{mg} / \mathrm{dL}$ for two consecutive days.

\subsubsection{Immunohistochemistry and Morphometric Analysis}

To determine hVEGF expression and angiogenesis, transplanted mice were sacrificed, kidneys were isolated, washed with PBS, fixed in $4 \%$ formaldehyde overnight, and embedded in paraffin as described before. ${ }^{133}$ Sections of 5-7 $\mu \mathrm{m}$ thickness were cut and immunostained with rabbit anti-insulin, hVEGF and hvW primary antibodies, respectively. The immunoreactivity was detected using goat anti-rabbit IgG, $\mathrm{H} \& \mathrm{~L}$ chain specific peroxidase conjugate and subsequent incubation with DAB substrate. For morphometric analysis, hVEGF and hvWF-positively immunostained color (brown in each case) was selected for quantification of the relative intensity using NIH Image 1.62 software to determine the mean values, which were subsequently compared between Adv-hVEGF-hIL-1Ra transduced and control islet transplanted diabetic recipient mice. 


\subsubsection{Statistical Analysis}

Statistical significance of the difference between the two groups was determined by unpaired t-test and between several groups by one-way ANOVA.

\subsection{Results}

The results of the study are discussed below.

\subsubsection{Construction of Adv-hVEGF-hIL-1Ra}

Adv-hVEGF-hIL-1Ra is a serotype 5 based replication deficient Adv vector that contains a CMV promoter, hVEGF cDNA and rabbit $\beta$-globin polyA in the E-1 region and a CMV promoter, hIL-1Ra cDNA and rabbit $\beta$-globin polyA in the E-3 region (Figure 2-1). Both cassettes are oriented from left to right relative to the adenovirus genome and lack the CMV intron that was present in the cosmid pADhVEGF-hIL-1Ra, from which no virus could be recovered. hVEGF and hIL-1Ra coding sequences and polyA signals were placed under the control of a separate CMV promoter cloned into $\mathrm{pE} 1.2$ and $\mathrm{pE} 3.1$. The resulting vectors were combined in Adenoquick 13.1 to generate a cosmid that contains the entire sequence of recombinant adenovirus. The presence of hVEGF and hIL-1Ra expression cassettes in these vectors was confirmed by restriction digestion with Hind III and NotI. The number of viral particles in the stock solution of Adv-hVEGF-hIL-1Ra was determined by $\mathrm{OD}_{260}-\mathrm{SDS}$ method. This gives the total number of $1.2 \times 10^{12}$ viral particles per $\mathrm{ml}$ of the stock solution irrespective of their plaque forming ability. The infectious titer of Adv-hVEGF-hIL-1Ra $\left(8 X 10^{10} \mathrm{IU} / \mathrm{ml}\right)$ was calculated by plaque assay method. This was further ascertained by performing the adenotiter assay using an Adeno X rapid titer kit and the titer was calculated to be 6 X10 $\mathrm{ifu} / \mathrm{ml}$ (infectious units/milliliter). We used infectious units/ml viral titer for all the experiments.

\subsubsection{Transduction Efficiency of Adv-hVEGF-hIL-1Ra}

Following transduction of islets with Adv-hVEGF-hIL-1Ra, the level and duration of hVEGF and hIL-1Ra proteins increased with increase in MOI. hVEGF and hIL-1Ra protein levels at day 3 post-transduction was over 100 times more than that of the untransduced islets (Figure 2-2), indicating that Adv-hVEGF-hIL-1Ra transduction would lead to high local concentrations of hVEGF and hIL-1Ra in the immediate vicinity of islets post transplantation. We also transfected islets with the bipartite plasmid DNA encoding these two genes (phVEGF-hIL-1Ra) after complex formation with Lipofectamine, which produced about 100 times lower levels of hVEGF and hIL-1Ra proteins than that of the islets transduced with Adv-hVEGF-hIL-1Ra (Figure 2-2).

We also determined hVEGF and hIL-1 Ra gene expression by real time RT-PCR. The 


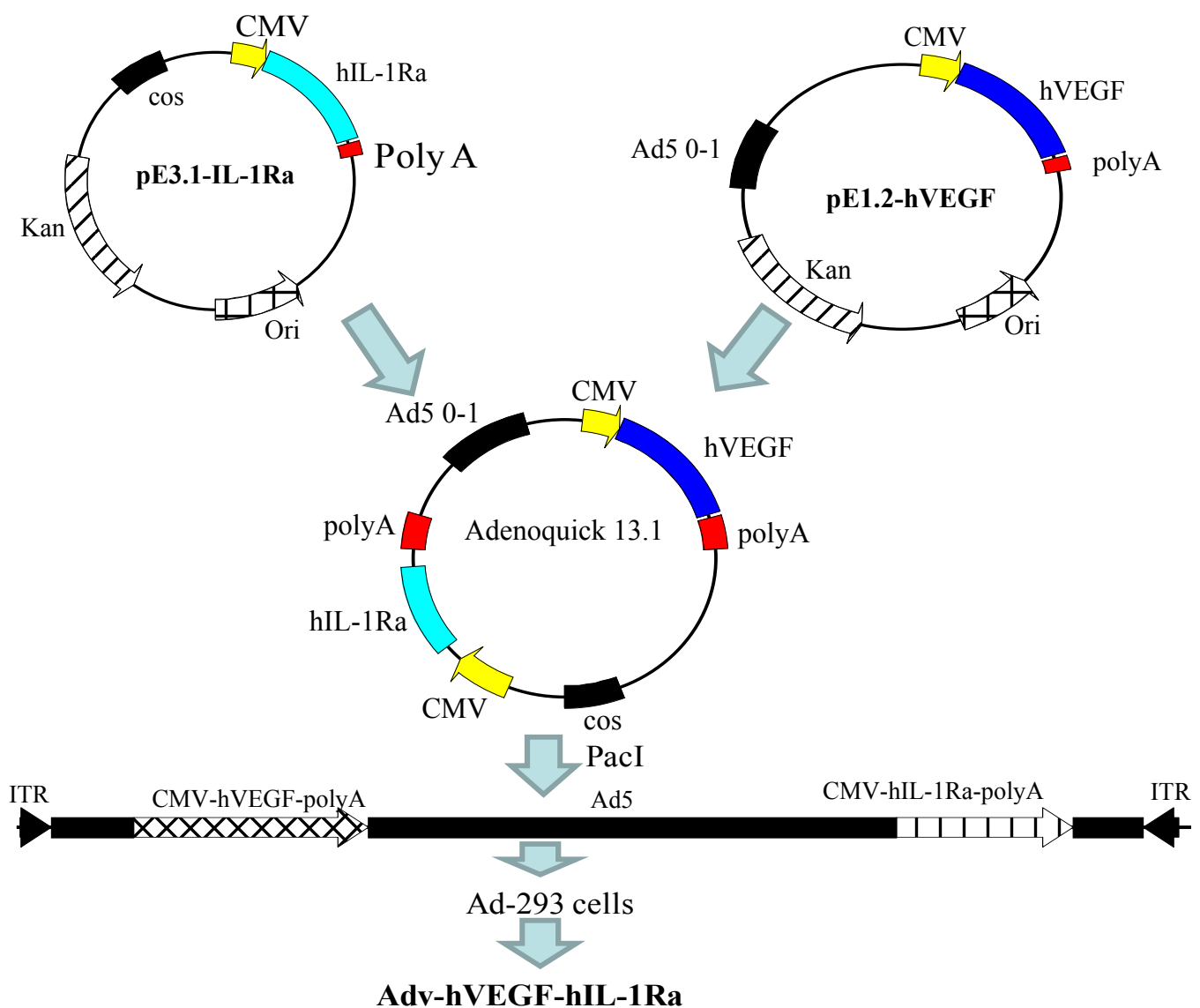

Figure 2-1 Construction of E1 and E3 deleted Adv-hVEGF-hIL-1Ra.

Construction of E1 and E3 deleted bipartite Adv vector by cloning hVEGF and hIL$1 \mathrm{Ra}$ into the multiple cloning sites of shuttle plasmids $\mathrm{pE} 3.1$ and $\mathrm{pE} 1.2$. These expression cassettes were cloned into AdenoQuick plasmid 13.1 to generate a cosmid containing the entire sequence of recombinant adenovirus. After transfection into 293 cells, AdvhVEGF-hIL-1Ra was produced. 

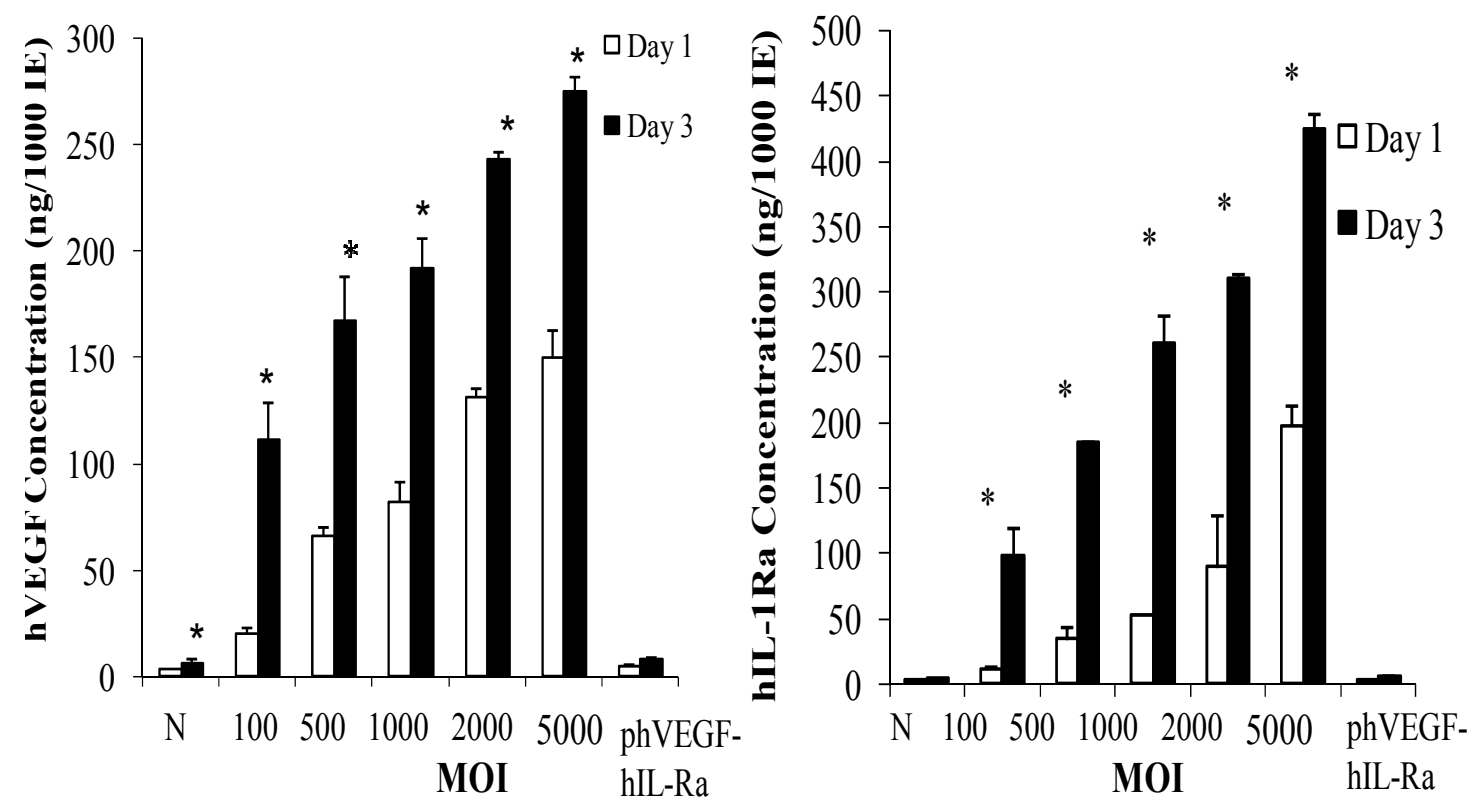

Figure 2-2 Time profile of hVEGF and hIL-1Ra at protein levels.

Time profile of hVEGF and hIL-1Ra gene expression at protein levels in human islets transduced Adv-hVEGF-IL-1Ra at different multiplicities of transduction (MOI) 100, $500,1000,2000$ and 5000 per 1000 islets. Non-transfected islets as well as the islets transfected with phVEGF-hIL-1Ra/Lipofectamine complexes $(3 / 1,+/-, 2.5 \mu \mathrm{g}$ DNA/1000 islets) were used as controls. ELISA of cell culture supernatants were performed at days 1 and 3. Cumulative expression levels were presented as the mean \pm S.D. of $n=4 .{ }^{*} p<0.05$ compared with untransduced islets using ANOVA. 
normalized mRNA concentration to that of the control was significantly increased with increase in MOI of Adv-hVEGF-hIL-1Ra (Figure 2-3). Gene expression at mRNA level of hVEGF and hIL-1Ra was more than 100 times higher than that of untransduced islets. Islets transfected with the bipartite plasmid, phVEGF-hIL-1Ra, also produced very low levels of hVEGF and hIL-1Ra mRNA. Each graph is indicative of the relative increase in expression of hVEGF and hIL-1Ra independently. This shows that Adv-hVEGF-hIL-1Ra can simultaneously express both genes with high efficiency. The mRNA levels of hVEGF and hIL-1Ra obtained by using one primer from the gene and the other from the Adv backbone showed dose dependent increase signifying that these genes were being expressed by Adv-hVEGF-hIL-1Ra (data not shown). The mRNA concentrations of each gene were normalized to that of the untransduced islets. The amplification specificity was confirmed by melting peak analysis, which showed a single predominant peak each for $18 \mathrm{~s}\left(85.4^{\circ} \mathrm{C}\right), \mathrm{hVEGF}\left(84.5^{\circ} \mathrm{C}\right)$ and $\mathrm{hIL}-1 \mathrm{Ra}\left(87.05^{\circ} \mathrm{C}\right)$, respectively (data not shown).

\subsubsection{Effect of Viral Transduction on Islet Function}

To determine whether insulin secretion was adversely affected by transduction of islets with Adv-hVEGF-hIL-1Ra, insulin secretion in response to glucose challenge was carried out at day 5 post-transduction. Glucose challenge was determined by quantifying glucose stimulated insulin release at basal level $(2.5 \mathrm{mM})$ and stimulated $(22 \mathrm{mM})$ glucose concentrations. As shown in Figure 2-4 stimulation index was almost identical to that of untransduced islets when 1000 islets were treated with Adv-hVEGF-hIL-1Ra at the dose of $1000 \mathrm{MOI}$. However, when 1000 islets were treated with this vector at $2000 \mathrm{MOI}$, there was some cytotoxicity resulting in decreased stimulation index.

\subsubsection{Apoptosis of Islets}

Apoptosis of islets leading to their impaired function and subsequent death is an important detriment to islet transplantation. Caspase-3 plays a major role in the apoptosis of islets. Therefore, we determined caspase-3 level in the islets transduced with AdvhVEGF-hIL-1Ra and incubated with a cocktail of inflammatory cytokines such as IL-1 $\beta$, TNF- $\alpha$ and IFN- $\gamma$. Caspase- 3 activity was very high in the un-transducted islets treated with the cytokine cocktail compared to that in the absence of the cytokine cocktail (Figure 2-5). The islets treated with 1000 and 2000 MOI Adv-hVEGF-hIL-1Ra and AdvhIL-1Ra showed significantly lower levels of caspase-3 activity compared to the untransduced control islets or the islets transducted with Adv-hVEGF at $1000 \mathrm{MOI}$ and incubated in the cytokine cocktail (Figure 2-5). In contrast, caspase-3 activity of all groups was very low when these islets were not incubated with the cytokine cocktail, suggesting that the Adv vector was non-toxic to islets at these tested doses.

We also determined the cytoprotective effect of Adv-hVEGF-hIL-1Ra on islet viability by fluorescence microscopy after transduction and incubation with the cytokine cocktail. These islets were stainied with propidium iodide, which is known to be 


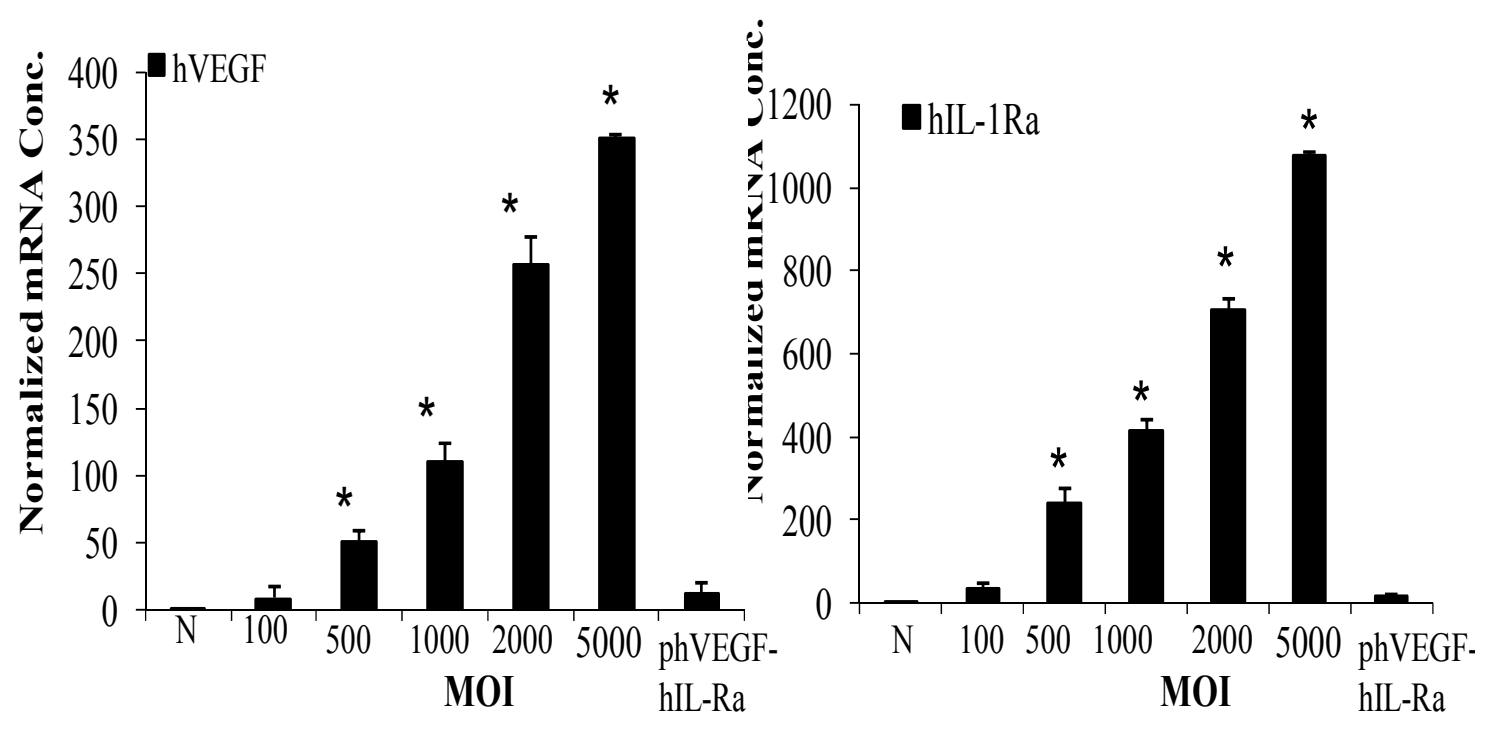

Figure 2-3 Real time PCR of islets transduced with Adv-hVEGF-IL-1Ra.

Real time PCR of human islets at day 3 post transduction with Adv-hVEGF-IL-1Ra at MOI of 100, 500, 1000, 2000 and 5000. Forward and reverse primers were designed from hIL-1Ra and hVEGF genes. Non-transfected islets as well as the islets transfected with phVEGF-hIL-1Ra/Lipofectamine complexes (3/1,+/-, $2.5 \mu \mathrm{g}$ DNA/1000 islets) were used as controls. Cell pellet was collected at day 3 and processed for mRNA extraction, reverse transcription and real time PCR using SYBR green chemistry. The normalized mRNA values of the different MOI treated islets are plotted. Since different primers were used, no direct comparison can be made, other than demonstration of increase in mRNA levels with increase in MOI. Results are expressed as the mean S.D of $n=4 * \mathrm{P}<0.05$ compared with control using ANOVA. 


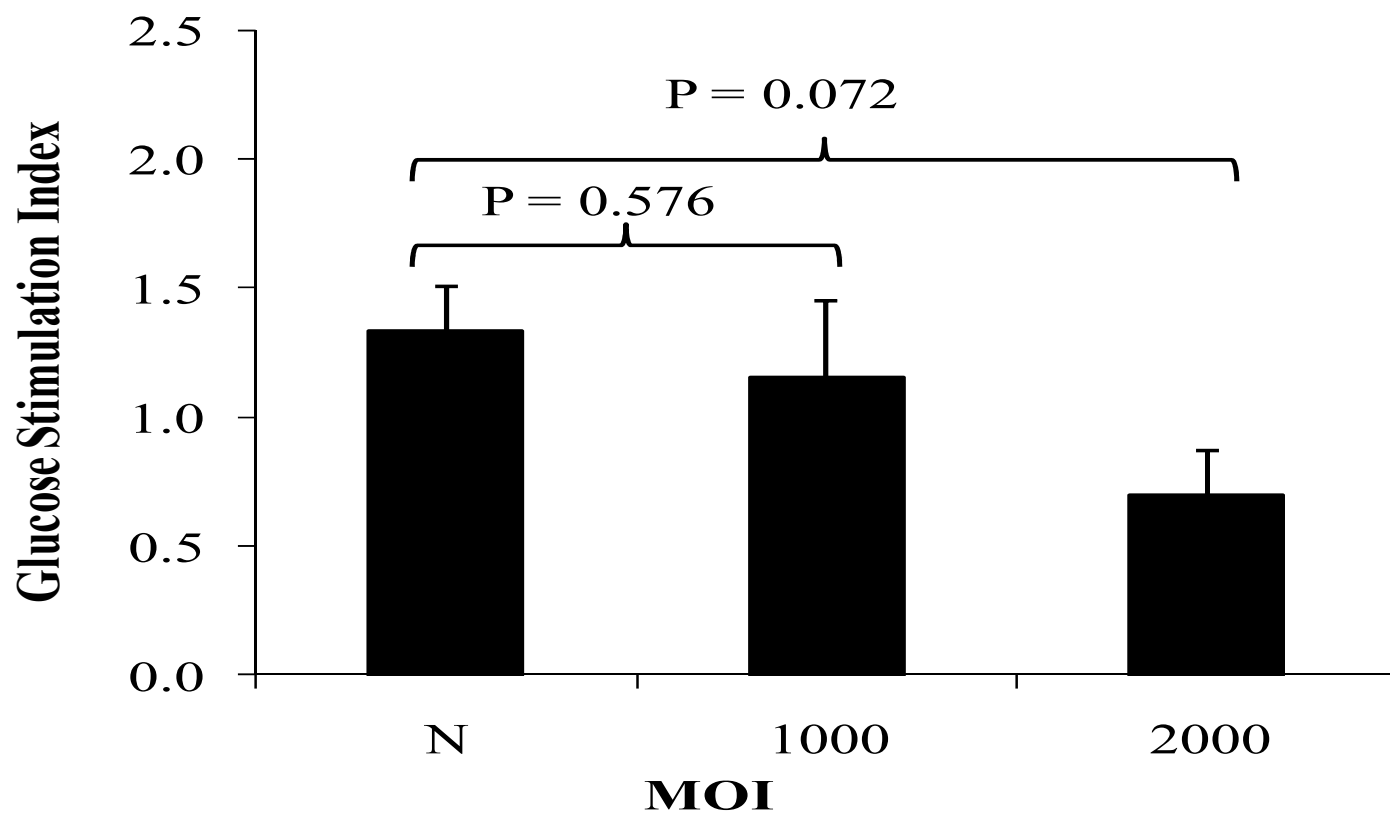

Figure 2-4 Glucose Stimulation Index.

Stimulation indexes (SI) of human islets with or without cytokine incubation after transduction with Adv-hVEGF-IL-1Ra. Non-transduced (N) islets were used as controls. Results are expressed as the mean \pm S.D of $n=3$. $*$ P value compared to untransduced islets. 


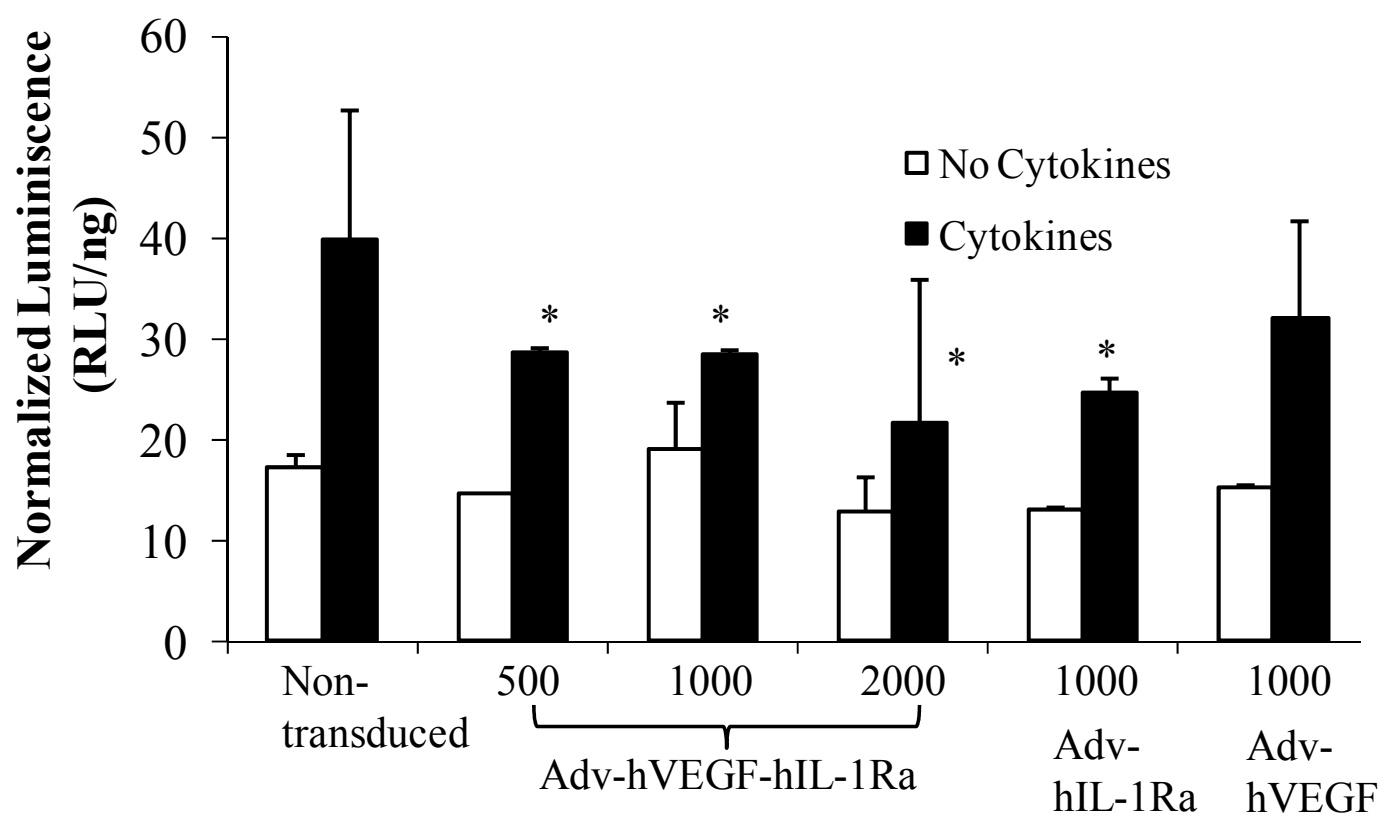

Figure 2-5 Effect of Adv-hVEGF-hIL-1Ra expression on caspase-3 activity.

Caspase-3 activity in human islets in the absence of or after incubation with the cytokines was determined by measuring the amount of caspase-3 present in the lysate of islets after cell lysis using a Caspase-Glo 3/7 luciferase assay. The islets were transduced with Adv-hVEGF-hIL-1Ra, at Adv-hVEGF or Adv-hIL-1Ra prior to incubating with cytokines IL-1 $\beta(10 \mathrm{ng} / \mathrm{ml})$, TNF- $\alpha(10 \mathrm{ng} / \mathrm{ml}) \&$ INF- $\gamma(50 \mathrm{ng} / \mathrm{ml})$. Non-transduced islets were used as control. Mean \pm S.D. of $n=3$, *p value compared with cytokine treated nontransduced islets using ANOVA. 
excluded by viable cells but can penetrate cell membranes of dying or dead cells, as evidenced red staining. As shown in Figure 2-6, the number of red cells was significantly lower in all the groups which were not incubated with the cytokine cocktail. However, incubation with the cytokine cocktail significantly increased the number of red cells in case of untransduced control islets. However, transduction of islets with Adv-hVEGFhIL-1Ra and Adv-hIL-1Ra significantly decreased the number of red cells even after incubation with the cytokine cocktail, suggesting the protective effect of hIL-1Ra gene expression. These results corroborate well with the results shown in Figure 2-5 for the effect of Adv-hVEGF-hIL-1Ra on caspase-3 activity.

\subsubsection{Islet Survival and Function after Transplantation}

The effect of hVEGF and hIL-1Ra gene expression on the islet survival and function post-transplantation of 1500 islets transduced with Adv-hVEGF-hIL-1Ra at $1000 \mathrm{MOI}$ under the kidney capsules of NOD-SCID mice was determined in terms of blood glucose, human insulin, and C-peptide levels. Diabetes was partly corrected in higher number of mice receiving Adv-hVEGF-hIL-1Ra transduced islets as compared to those receiving Adv-LacZ transfected and non-treated islets. Adv-hVEGF-AdvhIL-1Ra transduced islets showed much rapid reduction in blood glucose levels to $\leq 220 \mathrm{mg} / \mathrm{dl}$ ( 3 days) than AdvLacZ transduced or non-treated islets. Mice transplanted with Adv- hVEGF-hIL-1Ra transduced islets had the lowest blood glucose levels, followed by non-treated islets (Figure 2-7A). Blood glucose levels returned to $\geq 325 \mathrm{mg} / \mathrm{dL}$ upon removal of the islet graft-bearing kidney at days 21 after transplantation, confirming that transplanted islets were functional. Upon glucose challenge, both blood insulin and C-peptide levels were significantly higher in mice transplanted with Adv-hVEGF-hIL-1Ra transduced islets compared to all the other groups, indicating better islet engraftment and function (Figure 2-7B, C). These results suggest that compared to non-treated or Adv-LacZ transduced islets, co-expression of hVEGF and hIL-1Ra reduced the blood glucose level of mice, and increased the level of blood insulin and c-peptide upon glucose challenge.

\subsubsection{Immunohistochemistry}

To determine whether hVEGF gene expression after transduction with Adv-hVEGFhIL-1Ra will promote angiogenesis by human islets, we isolated the kidney bearing AdvhVEGF-hIL-1Ra transduced islets at 20 days post-transplantation into diabetic NODSCID mice for sectioning and staining with polyclonal rabbit hVEGF and vonWillebrand Factor (hvWF) antibodies. Staining with anti-human insulin and untransduced islet sections were used as the controls. The positive staining of hVEGF was higher in the transduced islets compared to untransduced islets (Figure 2-8), suggesting efficient hVEGF gene expression after transduction with Adv-hVEGF-hIL-1Ra. The positive staining of hvWF factor was more evident in Adv-hVEGF-hIL-1Ra transduced islets, which is an indicator for endothelial cells. Morphometric analysis revealed that AdvhVEGF-hIL-1Ra transduced Islet grafts exhibited higher hVEGF and hvWFimmunostaining and intensity than control islet grafts, with relative intensities of $1.45 \mathrm{vs}$. 


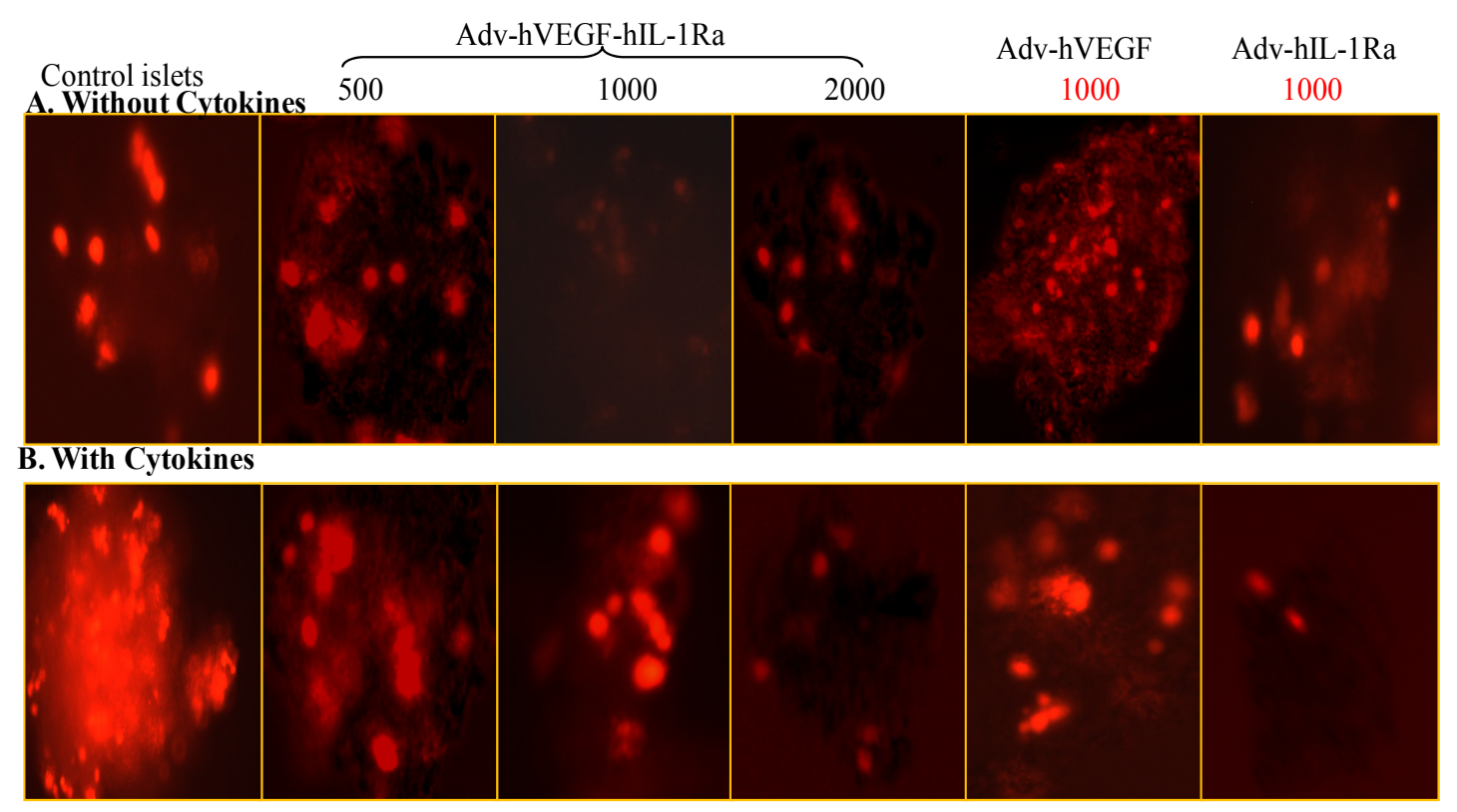

Figure 2-6 Effect of hVEGF and hIL-1Ra co-expression on islet viability.

Effect of hVEGF and hIL-1Ra co-expression on islet viability was examined under fluorescence microscopy after propidium iodide staining of intact islets transduced with Adv-hVEGF-hIL-1Ra, Adv-hIL-1Ra and Adv-hVEGF, and then incubated for 3 days with inflammatory cytokines. Red staining represents cell membranes of dying or dead cells. 

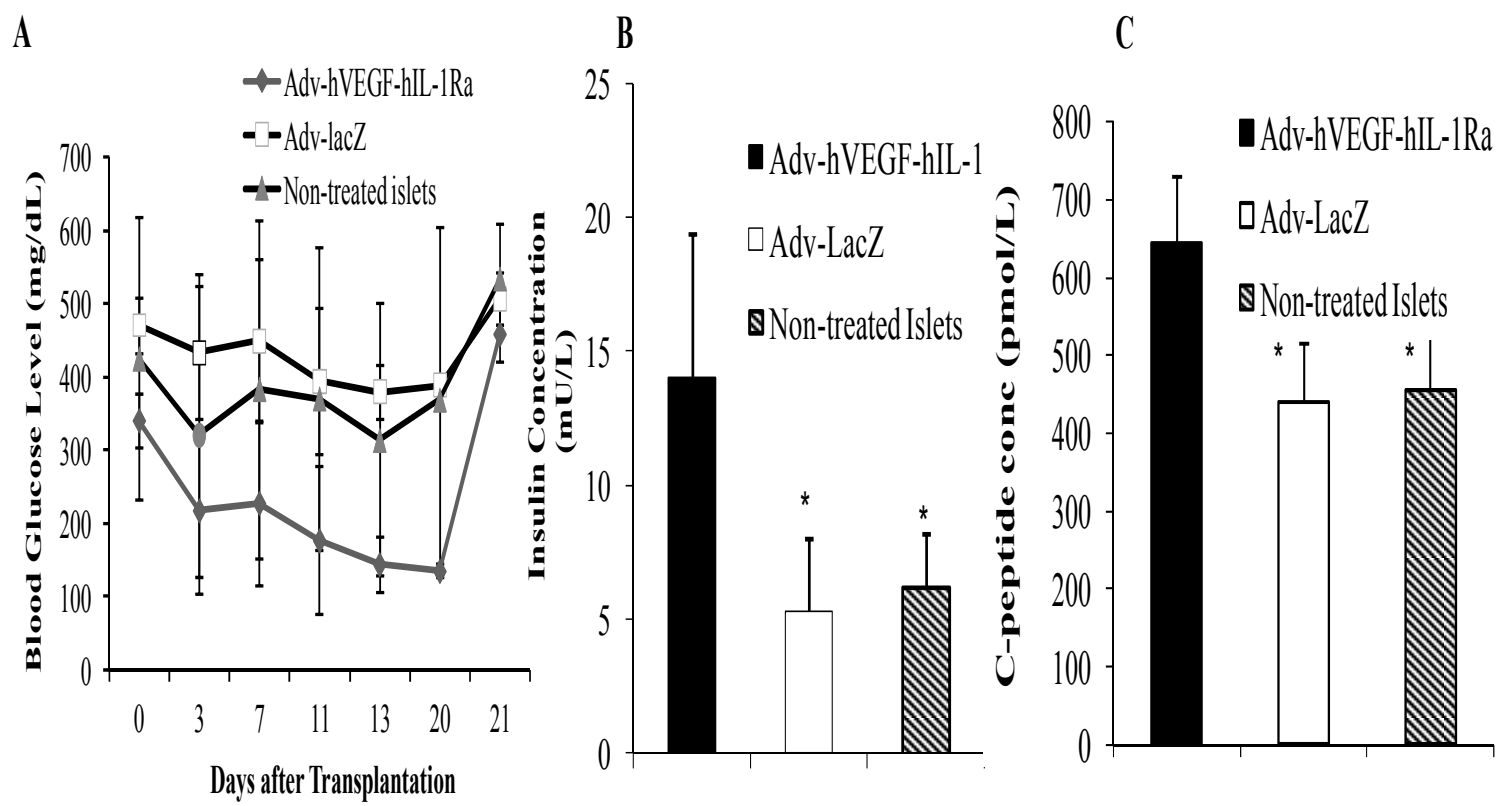

Figure 2-7 Transplantation of Adv-hVEGF-hIL-1Ra transduced islets.

Following transduction with Adv-hVEGF-hIL-1Ra at $1000 \mathrm{MOI}, 1500$ islets were transplanted under the kidney capsule of streptozotocin-induced diabetic NOD-SCID mice. The mice transplanted with Adv-LacZ-transduced and non-transduced islets were used as controls. A) The nonfasting glucose levels were measured in each animal up to 20 days post-transplantation using a One Touch Ultra Glucometer. Blood glucose levels returned to $\geq 325 \mathrm{mg} / \mathrm{dL}$ upon removal of the islet graft-bearing kidney at days 21 after transplantation. $\mathrm{B} \& \mathrm{C})$. At day 21 post-transplantation, the mice were sacrificed and blood was collected to measure serum insulin by ELISA and C-peptide by radioimmunoassay (RIA). Data are presented as the mean+S.D. of $n=4,{ }^{*} p<0.5$, compared to Adv-hVEGFhIL-1Ra transduced islet group. 

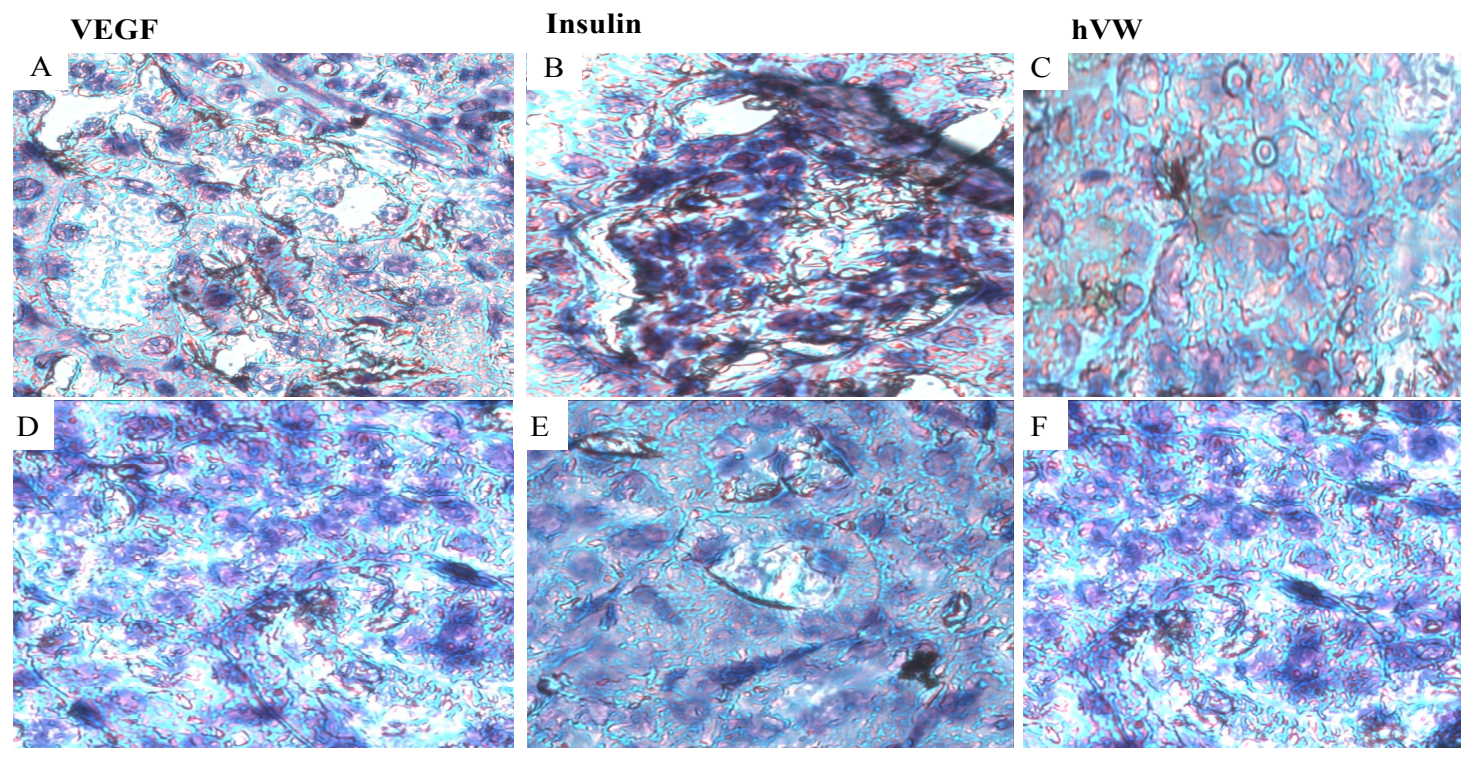

Figure 2-8 Immunohistochemical staining of islet bearing kidney sections.

Immunohistochemical staining of islet bearing kidney sections at day 20 posttransplantation of human islets transduced with Adv-hVEGF-hIL-1Ra at 1000 MOI.

Rabbit polyclonal anti human VEGF and hvW were used for detecting human VEGF and endothelial cells. These pictures were taken at $\times 40$ magnification. A) Insulin, B) hVEGF and C) vWF staining for the islets transduced with Adv-hVEGF-hIL-1Ra; and D) insulin, E) hVEGF and F) hvWF staining for the control islets. 
1 for hVEGF and 1.30 vs. 1 for hvWF, respectively. To correlate the degree of islet revascularization with the islet mass in the kidney capsule, we also stained for human insulin. Adv-hVEGF-hIL-1Ra transduced islets displayed significantly higher levels of insulin content than control islet groups (Figure 2-8B), with relative intensity of 1.3 vs. 1 . This quantitative difference in insulin content in the kidney capsule between AdvhVEGF-hIL-1 Ra transduced and control islet groups of diabetic recipient mice correlated well with their blood glucose, serum insulin and c-peptide profiles (Figure 2-7). The positive staining for human insulin also confirmed the functionality of islets. These results indicate the beneficial effect of Adv-hVEGF-hIL-1Ra for the islet survival and function post-transplantation.

\subsection{Discussion}

Isolation and purification of islets disrupt their microvasculature, leading to hypoxia and apoptosis upon transplantation. ${ }^{133} \mathrm{We}$ and others have shown that hVEGF expression promotes new blood vessel formation and improves the outcome of islet transplantation. ${ }^{18,133}$ However, effective prevention of islet destruction after transplantation requires not only revascularization but also abrogation of cytokine mediated islet cell death and dysfunction. Islet injury stimulates resident macrophages and infiltrating leukocytes to produce cytotoxic cytokines like IL-1 $\beta$, TNF- $\alpha$ and IFN- $\gamma$.

Among these cytokines, IL-1 $\beta$ is the key mediator of $\beta$ cell destruction. Administration of recombinant IL-1Ra protein has been shown to prevent islet dysfunction upon transplantation. ${ }^{151}$ Ex vivo transduction of human islets with Adv-hIL$1 \mathrm{Ra}$ has been reported to prevent IL-1 $\beta$ induced $\beta$ cell impairment and activation of islet cell apoptosis. ${ }^{150}$ Therefore, we recently transduced human islets with a mixture of AdvhVEGF and Adv-hIL-1 Ra ${ }^{133}$ and demonstrated suppression of cytokine induced iNOS gene expression and NO production. ${ }^{133,152}$ Similarly, Bertera et al., ${ }^{153}$ reported synergistic effect after simultaneous Adv transduction of mouse islets with two individual genes, such as IL-1Ra and indoleamine 2, 3 dioxygenase or hIL-1Ra and manganese superoxide dismutase (MnSOD).

The objective of this study was to evaluate a bipartite Adv vector for multiple gene delivery to human islets for promoting revascularization and inhibiting inflammatory cytokine-mediated destruction. There are several studies where bipartite Adv vectors have been constructed with one therapeutic gene and another marker gene, especially green fluorescence protein (GFP) ${ }^{154,155}$ In this study, we have constructed a replication deficient bipartite Adv vector encoding hVEGF and hIL-1Ra. The use of bipartite vector not only simplifies the amplification and purification process of Adv vectors, but also decreases the use of total Adv backbone. This is expected to minimize the immunogenic and toxic effects of Adv vectors. hVEGF is known to promote angiogenesis ${ }^{156}$ and improved vascularization can lead to reduced apoptosis. On the other hand, hIL-1Ra inhibits apoptosis of islets. ${ }^{151}$ Apoptosis begins during the islet isolation process, peaks 23 days post transplantation, and continues for approximately $7 \sim 14$ days until what remains of the graft has stabilized and become vascularized. Therefore, the use of 
replication deficient $\mathrm{Adv}$ vectors is appropriate. We decided to use $\Delta \mathrm{E} 1$ and $\Delta \mathrm{E} 3 \mathrm{Adv}$ vector because our goal was to achieve transient gene expression, while avoiding other potential side effects and in vivo virus replication. To determine hVEGF and hIL-1Ra gene expression, we first transduced human islets with Adv-hVEGF-hIL-1Ra and then monitored transgene expression for 3 days. hVEGF and hIL-1Ra expression over days 1 and 3 showed considerable increase (Figure 2-2), suggesting the efficacy of Adv-hVEGFhIL-1Ra. Variation in MOI, promoters and duration of the incubation time are known to affect the transfection efficiency of Adv vectors in various cell types. Therefore, we sought to optimize the transduction conditions of human islets with Adv-hVEGF-hIL1Ra. hVEGF and hIL-1Ra gene expression increased with increase in MOI from 100 to 5000. However, we decided to infect 1000 islets with this vector at $1000 \mathrm{MOI}$ to avoid any toxic side effects. Unlike the Adv vector, transfection with the bipartite plasmid encoding hVEGF and hIL-1Ra (p-hVEGF-hIL-1Ra) produced very little hVEGF and hIL-1Ra (Figure 2-2) which is consistent with our previous results ${ }^{18}$ and is expected since an islet is a cluster of 200-1000 cells and difficult to transfect.

Since prolonged incubation with Adv vectors may induce apoptosis and loss of endothelial cells; we decided not to co-culture the islets beyond $12 \mathrm{~h}$. We then determined $\mathrm{hVEGF}$ and hIL-1Ra gene expression at mRNA level for which we used $18 \mathrm{~s}$ as an internal control. The mRNA levels of hVEGF and hIL-1Ra increased with increase in MOI (Figure 2-3), indicating the Adv vector is indeed expressing these two genes as desired. Although hVEGF and hIL-1Ra increased at both protein and mRNA levels with increase in the Adv MOI, these results cannot be directly correlated. Unlike ELISA, we used different primers for different genes (in this case hVEGF and hIL-1Ra) and thus we can only compare the results relative to the house keeping genes. Also, it is known that there is a poor correlation between mRNA and protein levels. That is why we have to use house-keeping genes whenever we do real time PCR. Jansen et al., have hypothesized that low correlation of protein and mRNA levels are a result of post translational modifications. ${ }^{157}$

Transduction process should not affect the normal insulin secretion capacity of human islets, which varies from batch to batch. Therefore, we measured insulin release in response to glucose challenge to assess the islet functional viability. As shown in Figure 2-4, there was little decrease in the stimulation index when islets were transduced with Adv-hVEGF-hIL-1Ra at the dose of 1000 MOI per 1000 islets. These data indicate that transduction with Adv-hVEGF-hIL-1Ra had only minimal adverse effect on insulin producing $\beta$ cells of the islets in response to glucose challenge.

Several studies have shown little induction of inflammatory responses on islet apoptosis when islets are transduced with Adv vectors at low MOI, which is also true in our studies. Since we wash islets with PBS to remove any free viral particles prior to transplantation and these vectors being replication deficient, we do not expect the host to mount a rigorous immune response. While there will be no free viral particles with the transplanted islets, even direct administration of this dose of Adv vectors have been seen to be non-toxic. Studies of Crystal and coworkers further showed local delivery of low 
and intermediate doses of replication deficient Adv vectors $\left(10^{11}\right.$ particles $)$ well tolerated. $^{158}$

Most islet grafts are destroyed due to immune and inflammatory reaction mediated by proinflammatory cytokines. To mimic the in vivo situation of the cytokine mediated insults we incubated islets with a cocktail of IL-1 $\beta$, TNF- $\alpha$ and INF- $\gamma$. Incubation with the inflammatory cytokines induced apoptosis in islets indicated by the increase in caspase-3 (Figure 2-5), which is a converging point of apoptosis for intrinsic and extrinsic pathways and caspase- 6 and 7 are generated from caspase- 3 . However, the islets treated with 1000 and 2000 MOI Adv-hVEGF-hIL-1Ra and Adv-hIL-1Ra showed significantly lower levels of caspase-3 activity compared to the untransduced islets or the islets transducted with Adv-hVEGF at $1000 \mathrm{MOI}$ and incubated in the cytokine cocktail, possibly due to hIL-1Ra gene expression (Figure 2-5). Similarly, incubation of islets with the cytokine cocktail significantly increased the number of red cells indicating dying or dead cells (Figure 2-6), which was much less for the islets transduced with Adv-hVEGFhIL-1Ra or Adv-hIL-1Ra, even after incubation with the cytokine cocktail. These results are well correlated with the literature where Adv vector encoding IL-1 Ra ${ }^{150}$ as well as recombinant IL-1 $\mathrm{Ra}^{159}$ have shown to protect islets from inflammatory cytokines. In contrast, there was no increase in caspase-3 after transduction of islets with Adv-hVEGFhIL-1Ra, Adv-hIL-1Ra or Adv-hVEGF in the absence of inflammatory cytokines, suggesting these Adv vectors are fairly safe at these tested doses.

Effect of Adv-hVEGF-hIL-1Ra transduction on improvement in the survival and function of islets posttransplantation was determined in streptozotocin-induced diabetic NOD-SCID mice. We observed a reduction in blood glucose levels of mice transplanted with Adv-hVEGF-hIL-1Ra transduced islets than that observed with Adv-LacZtransduced or non-treated islets (Figure 2-7A). This increase correlated with the higher amount of serum insulin and C-peptide secreted by the islets at day 20 posttransplantation (Figure 2-7B and C). These results indicate that hVEGF and hIL-1Ra coexpression is likely to reduce the islet mass required to achieve normoglycemia.

Although we did not examine the apoptosis in graft islet cells, our in vitro data clearly indicated transduction of Adv-hVEGF-hIL-1Ra led to significant reduction of caspase-3 activity, which is the indicator of apoptosis.

Revascularization of islet grafts after transplantation is an important process that influences long-term survival and function of the grafts. Endothelial cells reside within isolated islets and potentially participate in the revascularization process. Immunohistochemical staining in the islet bearing kidney sections at day 20 after transplantation was positive for human insulin, hVEGF and hvWF (Figure 2-8). Positive staining for insulin, which stains pancreatic $\beta$-cells, corroborates the blood insulin data and confirms that islets are indeed functional after transplantation, which is in good agreement with the contemporary literature. hvWF is human endothelial cell marker. Therefore, relatively stronger positive staining for hVEGF and hvWF antigens by the Adv-hVEGF-hIL-1Ra transduced islet bearing kidney section groups indicates that blood vessel formation in the transplanted islets was relatively more efficient when islets were transduced with Adv-hVEGF-hIL-1Ra prior to transplantation. This indicates the 
usefulness of this adenoviral vector in improving the survival and function of islets after transplantation. 


\section{CHAPTER 3. BIPARTITE ADENOVIRAL VECTOR ENCODING hHGF AND hIL-1Ra FOR IMPROVED HUMAN ISLET TRANSPLANATATON}

\subsection{Introduction}

Type I diabetes is a chronic inflammatory autoimmune disease resulting from the loss of islet function, requiring life-long insulin therapy. Human islet transplantation has the potential to replace pancreatic endocrine function in patients with Type I diabetes ${ }^{160,161}$ One major drawback to this approach is the requirement of islets derived from two to four cadaver donors to treat a single patient with diabetes. In addition, required islet mass has been seen in successfully transplanted recipients compared with healthy people ${ }^{162}$ Thus, an enormous shortage of human islets is a barrier to the use of islet transplantation on a larger scale. Despite recent success, islet transplantation still lags behind primarily because a large number of transplanted islets often do not function. ${ }^{143}$

Islets have an intricate microvasculature, which is disrupted during islet isolation and purification process. Therefore, revascularization to the transplanted islets is essential for delivery of nutrients and oxygen to the inner core of islets for their survival. ${ }^{100,133,144,163}$ Hepatocyte growth factor (HGF) is a potent mitogen for human islets and is known to increase the proliferation and expansion of $\beta$-cells and promotes revascularization of islets, while maintaining physiologic glucose responsiveness after transplantation. ${ }^{104}$ Overexpression of HGF in the $\beta$-cells of transgenic mice directed by the rat insulin Type II promoter (RIP) has shown to increase $\beta$-cell mass and proliferation, and improve islet function and islet transplant outcome. ${ }^{164}$ Ex vivo transduction of islets with an adenoviral $(\mathrm{Adv})$ vector encoding HGF has been reported to improve the outcome of islet transplantation. ${ }^{165}$ Intravenous injection of Adv-HGF in streptozoticin (STZ)-induced diabetic mice have been shown to ameliorate hyperglycemia and prolong survival period in the diabetic mice. ${ }^{166}$ Moreover, HGF gene transfer has also been shown to increase the expression of three key $\beta$-cell genes, glucokinase, Glut-2 (the $\beta$-cell glucose transporter), and insulin. ${ }^{167}$

Success of islet transplantation not only depends on islet revascularization, but also on its protection from apoptosis induced by hypoxia, oxidative stress and inflammatory cytokines released from resident macrophages and infiltrating immune cells at the transplantation site. ${ }^{147}$ More than $70 \%$ of islets become inactive in 7-14 days posttransplantation. ${ }^{168}$ Among these cytokines, IL-1 $\beta$ plays a key role in apoptotic cell death. Inhibition of IL-1 $\beta$ receptor binding using interleukin-1 receptor antagonist (IL-1Ra) resulted in improved islet survival and function. ${ }^{149-151} \mathrm{HGF}$ has also been reported to induce resistance to apoptosis. ${ }^{169}$

Considering the favorable uses of hHGF and hIL-1Ra, in this study we constructed a bipartite Adv vector encoding these two genes (Adv-hHGF-hIL-1Ra) by cloning hIL-1Ra and hHGF under a separate cytomegalovirus (CMV) promoter. We determined transduction efficiency, islet function, and caspase-3 release upon incubation with inflammatory cytokine cocktail of TNF- $\alpha$, IL-1 $\beta$ and IFN- $\gamma$. We then transplanted islets 
under the kidney capsules of nonobese diabetic/severe combined immunodeficient (NOD-SCID) mice after transduction with Adv-hHGF-hIL-1Ra and determined the levels of blood glucose and serum insulin. Finally, the islet bearing kidney sections of these diabetic mice were stained for human insulin, hHGF and von Willebrand factor (vWH).

\subsection{Materials and Methods}

\subsubsection{Materials}

Agarose, tris-borate-EDTA buffer, goat serum, glucose and haematoxylin solution were purchased from Sigma-Aldrich Co. (St. Louis, MO). Fetal bovine serum (FBS) and CMRL-1066 media were purchased from MediaTech Cellgros (Herndon, VA). Phosphate buffer solution (PBS) was purchased from GIBCO-BRL (Gaithersburg, MD). Human HGF and IL-1Ra ELISA kits and cytokines IL-1 $\beta$, TNF- $\alpha$ and IFN- $\gamma$ were purchased from R\&D Systems (Minneapolis, MN). Human Insulin ELISA kits were purchased from Alpco Diagnostics (Windham, NH). RNA extraction kit and Caspase-Glo 3/7 assay kit were procured from Promega (Madison, WI). SYBR Green real time PCR master mix and reverse transcription reagents were purchases from Applied Biosystems (Foster City, CA). Sodium citrate was procured from Curtin Matheson Science Inc. (Houston, TX.)

\subsubsection{Methods}

The following methods were used with regards to generation of the adenoviral vector and testing its efficacy both in vitro and in vivo.

\subsubsection{Construction of Adv-hHGF-hIL-1Ra}

A bipartite Adv vector expressing hHGF and hIL-1Ra genes was constructed with the help of OD 260, Inc. (Boise, ID). Briefly, the hHGF cDNA was excised from pCMVhHGF using BamHI, and cloned into the BamHI site of $\mathrm{pE1}$.2, which contains a CMV promoter, multiple cloning site, and bGH polyA signal. Similarly, hIL-1Ra cDNA was excised from pUMVC-hIL-1Ra vector using ScaI, separated by gel fractionation, and purified using Promega PCR and cleanup kit (Promega, Madison, WI). The hIL-1Ra fragment along with rabbit $\beta$-globin polyA was then cloned into $\mathrm{pE3} .1$ shuttle plasmid using NotI restriction site. The $\mathrm{pE}$ 3.1 IL-1Ra plasmid was further modified by removing the intron adjoining CMV promoter. All the ligation reactions were done at 1:3 and 1:10 ratios using T4 DNA ligase and then transformed in super competent TOP 10 cells and amplified in terrific broth media. The plasmids were then purified using Gen Elute plasmid Mini prep kit (Sigma-Aldrich, St. Louis, MO). The plasmids pE 1.2-hHGF and pE 3.1-hIL-1Ra were digested with AlwNI and DraIII respectively and the excised fragments were purified on agarose gel. These fragments were ligated with SfiI-digested AdenoQuick 13.1 plasmid simultaneously using T4 DNA ligase overnight at $16^{\circ} \mathrm{C}$. The 
ligated products were packaged into phage lambda particles using MaxPlax packaging extract (Epicentre, Madison, WI). The packaging mixture was used to infect E. coli XL1Blue (Stratagene) and clones were selected on LB supplemented with $25 \mu \mathrm{g} / \mathrm{ml}$ kanamycin and $50 \mu \mathrm{g} / \mathrm{ml}$ ampicillin. The Pac I linearized cosmid was transfected into 293 cells to rescue the virus. Three viral plaques were amplified, and their genomic DNAs were extracted. The presence of hHGF and hIL-1Ra expression cassettes in these three vectors was confirmed by restriction analysis with SpeI and EcoRV, respectively. The clone containing the two genes was infected to 293 cells. Both the cells and the medium were harvested and frozen/thawed three times. This viral extract was further infected into 293 cells, amplified and purified on two $\mathrm{CsCl}$ gradients. The bands harvested were dialyzed against $3 \mathrm{X} 11 \mathrm{GTS}$ buffer (25\% glycerol, $25 \mathrm{mM} \mathrm{NaCl}, 20 \mathrm{mM}$ Tris-HCl (pH 8) for 12 hours at $4^{\circ} \mathrm{C}$. The dialyzed viral suspension was collected, filtered and stored at $-70^{\circ} \mathrm{C}$.

\subsubsection{Determination of Viral Titer}

The infectious viral titer was also determined by using an Adeno-X rapid titer kit from Clontech (Mountain View, CA). Different dilutions of Adv-HGF-hIL-1Ra was taken and transduced to AD-293 cells, using un-transduced cells as a control. After 48h post-transduction, AD-293 cells were fixed and incubated with Anti-Hexon antibody, followed by a secondary horse radish peroxidase (HRP)-conjugated antibody. Then the cells were stained with 3, 3' diaminobenzidine tetrahydrochloride (DAB) substrate and positively stained cells were counted under a microscope, and the titer was determined.

\subsubsection{Islet Culture and Transduction}

Human Islets were received from one of the several Islet Cell Resource (ICR) Centers in culture media at $4^{\circ} \mathrm{C}$. Upon receipt, the islets were cultured in CMRL-1066 medium containing 10\% fetal bovine serum (FBS) and 1\% Penicillin Streptomycin. Islets were then transduced with Adv hHGF-hIL-1Ra, while non-transduced islets were used as controls. To determine the optimal multiplicity of Infection (MOI) of Adv in terms of plaque forming units (pfu) per islet equivalent, 1000 islets were incubated with diluted adenovirus at 100, 500, 1000, 2000 and $5000 \mathrm{MOI}$ in $300 \mu$ media in a 96 well plate for $12 \mathrm{~h}$, followed by washing and further culture in $1 \mathrm{ml}$ of media in $24-w e l l$ plates. Gene expression was determined at days 1 and 3 by quantifying secreted protein in the media by ELISA (R\&D Systems, Minneapolis, MN). Total protein of the islet cell extracts was also determined using the bicinchoninic acid (BCA) protein assay kit to normalize the results. Three samples per group were included for the experiment.

\subsubsection{Quantitative Real Time PCR}

To determine hHGF and hIL-1Ra gene expression in human islets at the mRNA level by real time RT-PCR, at day 3 post-transduction of islets with Adv-hHGF-hIL-1Ra the 
islet pellet was collected for isolation of total RNA using RNAeasy mini isolation kit from Promega. RNA concentration was measured by UV spectrophotometry using Biomate 3 spectrophotometer. Two hundred and fifty nanograms (ng) of extracted RNA was converted into cDNA using MultiScribe reverse transcriptase and random hexamers (Applied Biosystems, Inc., Foster City, CA) by incubation at $25^{\circ} \mathrm{C}$ for $10 \mathrm{~min}$, followed by reverse transcription at $48^{\circ} \mathrm{C}$ for $30 \mathrm{~min}$ and enzyme inactivation at $95^{\circ} \mathrm{C}$ for $5 \mathrm{~min}$. In all $3.85 \mu \mathrm{l}$ of cDNA was amplified by real time RT-PCR. To determine the level of HGF expression, the following hHGF-specific primers were used: Forward: 5'-CCT ATG CAG AGG GAC AAA GGA A-3'; Reverse: 5'-GCC TTG CAA GTG AAT GGA AGT CCT-3' (NCBI Accession\#291674). In case of hIL-1Ra both, forward (5'-ATC CAG CAA GAT GCA AGC CCT-3') and reverse (5'-TTC GTC AGG CAT ATT GGT GAG GCT-3') primers were designed from the gene sequence, (NCBI Accession\#AK290898). The PCR conditions included denaturation at $95^{\circ} \mathrm{C}$ for $10 \mathrm{~min}$, followed by 40 cycles of amplification by sequential denaturation at $95^{\circ} \mathrm{C}$ for $15 \mathrm{sec}$ and primer annealing as well as strand extension for $1 \mathrm{~min}$. $\mathrm{hHGF}$ and hIL-1Ra gene expression was normalized to $19 \mathrm{~s}$ rRNA as internal control.

To confirm the amplification specificity, the PCR products were subjected to melting curve analysis. The expressed HGF and hIL-1Ra levels were quantified and normalized to the total amount of cDNA used. Threshold cycle number was compared between AdvhHGF-hIL-1Ra with the same parameters using non-transfected islets as controls.

\subsubsection{In Vitro Islet Function after Infection}

The in vitro function of human islets after infection with Adv-hHGF-hIL-1Ra was determined by the static incubation method. Non-infected islets were used as control. Transfected media was removed by centrifugation at $300 \mathrm{~g}$ and the islets were sequentially incubated at $37^{\circ} \mathrm{C}$ for $1 \mathrm{~h}$ in the media containing $2.5 \mathrm{mM}$ (basal) and $22 \mathrm{mM}$ glucose (stimulated). After incubation, supernatants were collected and analyzed for insulin release by ELISA (Alpco Diagnostics). Insulin secretion was expressed as $\mu \mathrm{U} / \mathrm{ml}$ and the ratio of insulin levels at $22 \mathrm{mM}$ to $2.5 \mathrm{mM}$ glucose was used to calculate the stimulation index.

\subsubsection{Cytoprotective Effect of Adv-hHGF-IL-1Ra}

One thousand islets per well of 96 well plates were infected with $1000 \mathrm{MOI}$ of AdvHGF-hIL-1Ra at 500, 1000 and 2000 MOIs. At 12h post-transduction, islets were washed and incubated with a cocktail of IL- $\beta(10 \mathrm{ng} / \mathrm{ml})$, TNF- $\alpha(10 \mathrm{ng} / \mathrm{ml})$ and IFN- $\gamma(50 \mathrm{ng} / \mathrm{ml})$ cytokines. Non-transduced islets with and without cytokine treatment were used as controls. Blank media was also used the negative control. At day 3 post transduction islets were collected from each well and were lysed with lysis buffer to measure the caspase-3 concentration using the Caspase-Glo 3/7 Assay kit (Madison, WI). 


\subsubsection{Western Blotting}

At day 3 post-transduction of human islets with Adv-hHGF-hIL-1Ra, protein extracts were prepared by washing cells with PBS and lysing in RIPA buffer containing protease inhibitor (Roche, Basel, Switzerland). Human islets were homogenized and lysates were spun at $15000 \mathrm{~g}$ for $15 \mathrm{~min}$ to remove insoluble material. Protein concentrations were determined by BCA protein assay. Proteins were resolved on 4-15\% sodium dodecyl sulfate polyacrylamide gel electrophoresis (SDS-PAGE) (Bio-Rad, Hercules, CA) and transferred to a PVDP membrane (Bio-Rad, Hercules, CA), blocked with TBST buffer composed of $50 \mathrm{mM}$ Tris $(\mathrm{pH} 7.6), 150 \mathrm{mM} \mathrm{NaCl}$ and $0.05 \%$ Tween supplemented with $5 \%$ fat-free milk at $4{ }^{\circ} \mathrm{C}$ for $1 \mathrm{~h}$. After a brief rinse, the membrane was incubated overnight at $4^{\circ} \mathrm{C}$ in TBST containing $0.5 \%$ milk with mouse anti-human Bcl-2 (Santa Cruz, CA) (1:200) and mouse anti-human Bax (Santa Cruz, CA). Goat anti-human Actin monoclonal antibody (Santa Cruz, CA) (1:2000) was used for detecting an internal control protein. Then, blots was washed four times using TBST for $1 \mathrm{~h}$ at room temperature. Immunodetection was performed using a horseradish peroxidase (HRP) Chemi Blot Starter Kit (Bio-rad, Hercules, CA) according to the manufacture's instruction.

\subsubsection{Islet Transplantation Studies}

Animal experiments were performed as per the $\mathrm{NIH}$ (http:/grants1.nih.gov/grants/olaw/ references/phspol.htm) and institutional animal care and use guidelines using the approved protocol. To induce diabetic animal model, streptozotocin (STZ) $(40 \mathrm{mg} / \mathrm{kg})$ was administered to NOD-SCID mice by intraperitoneal injection for 5 consecutive days. Animals were considered to be diabetic after two consecutive measurements of blood glucose $\geq 300 \mathrm{mg} / \mathrm{dl}$ using a One Touch Ultra Glucometer (LifeScan, Inc.). Before transplantation, human islets were transduced by Adv-hHGF-hIL-1Ra at the dose of 1000 MOI for 12h and washed with PBS. About 1000 transduced or non-treated islets were transplanted under the left kidney capsules of diabetic mice. The nonfasted glucose levels were measured from the snipped tail of each animal up to 28 days post-transplantation. At day 28 post-transplantation, the mice were then anesthetized to collect blood to measure serum insulin and c-peptide levels by ELISA (Alpco Diagnostics, Salem, NH). At day 28, the graft-bearing kidneys were removed from some animals to confirm the function of islet grafts by the return of blood glucose levels to $\geq 300 \mathrm{mg} / \mathrm{dL}$ for two consecutive days.

\subsubsection{Intraperitoneal Glucose Tolerance Test}

Twenty seven days after islet transplantation under the kidney capsules of STZinduced NOD-SCID diabetic mice, glucose tolerance was analyzed in overnight fasted mice by intraperitoneal injection of $2 \mathrm{~g}$ of glucose $/ \mathrm{kg}$ of body weight as described by Garcia-Ocana et al. ${ }^{165}$ Blood samples were obtained from the snipped tail and analyzed for glucose levels using a One Touch Ultra Glucometer (LifeScan, Inc.). 


\subsubsection{Immunohistochemistry and Morphometric Analysis}

To determine hHGF expression and angiogenesis, transplanted mice were sacrificed, kidneys were isolated, washed with PBS, fixed in 4\% formaldehyde overnight, and embedded in paraffin as described before. ${ }^{133}$ Sections of $5-7 \mu \mathrm{m}$ thickness were cut and immunostained with rabbit anti-insulin, hHGF and hvW primary antibodies, respectively. The immunoreactivity was detected using goat anti-rabbit IgG, H\&L chain specific peroxidase conjugate and subsequent incubation with DAB substrate. For morphometric analysis, hHGF and hvWF-positively immunostained color (brown in each case) was selected for quantification of the relative intensity using NIH Image 1.62 software to determine the mean values, which were subsequently compared between Adv-hHGF-hIL$1 \mathrm{Ra}$ transduced and control islet transplanted diabetic recipient mice.

\subsubsection{Statistical Analysis}

Statistical significance of the difference between the two groups was determined by unpaired t-test and between several groups by one-way ANOVA.

\subsection{Results}

The results obtained from these studies are outlined below.

\subsubsection{Construction of Adv-hHGF-hIL-1Ra}

Adv-hHGF-hIL-1Ra is a serotype 5 based replication deficient Adv vector that contains a CMV promoter, hHGF cDNA and rabbit $\beta$-globin poly $\mathrm{A}$ in the $\mathrm{E}-1$ region and a CMV promoter, hIL-1Ra cDNA and rabbit $\beta$-globin poly $\mathrm{A}$ in the E-3 region (Figure 3-1). hHGF and hIL-1Ra coding sequences and polyA signals were placed under the control of a separate CMV promoter cloned into $\mathrm{pE} 1.2$ and $\mathrm{pE} 3.1$. The resulting vectors were combined in Adenoquick 13.1 to generate a cosmid that contains the entire sequence of recombinant adenovirus. The presence of hHGF and hIL-1Ra expression cassettes in these vectors was confirmed by restriction digestion with Hind III (data not shown). The number of viral particles in the stock solution of Adv-hHGF-hIL-1Ra was determined using an Adeno X rapid titer kit from Clontech (Mountain View, CA) and the titer was calculated to be $1.24 \mathrm{X} 10^{\wedge} 9 \mathrm{ifu} / \mathrm{ml}$ (infectious units $/ \mathrm{ml}$ ).

\subsubsection{Transduction Efficiency of Adv-hHGF-hIL-1Ra}

Following the transduction of human islets with Adv-hHGF-hIL-1Ra, the level and duration of hHGF and hIL-1 Ra proteins increased with increase in MOI. hHGF and hIL1 Ra protein levels at day 3 post-transduction was over 60 and 40 times higher than that of the untransduced islets, respectively (Figure 3-2), indicating that Adv-hHGF-hIL-1Ra 

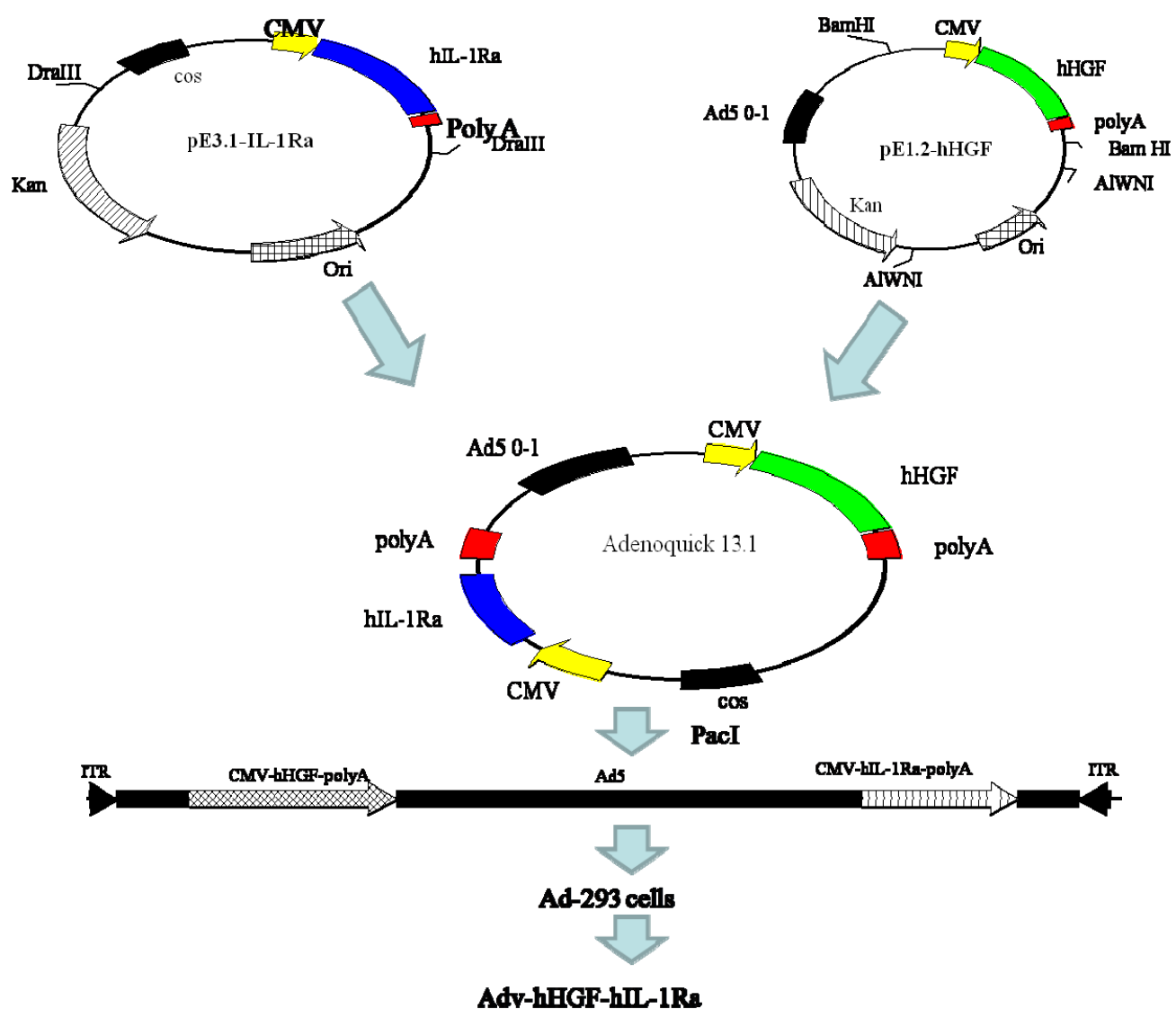

Figure 3-1 Construction of E1 and E3 deleted bipartite Adv-hHGF-hIL-1Ra.

Adv-HGF-hIL-1Ra was constructed by excising hHGF and hIL-1Ra form vector pUMVC and cloning into the multiple cloning sites of shuttle plasmids $\mathrm{pE} 3.1$ and $\mathrm{pE} 1.2$ using NotI. The expression cassettes of hHGF and hIL-1Ra containing the CMV promoter were cloned into AdenoQuick plasmid 13.1 to generate a cosmid containing the entire sequence of recombinant adenovirus. After transfection into 293 cells, recombinant adenovirus Adv-hHGF-hIL-1Ra was produced. 


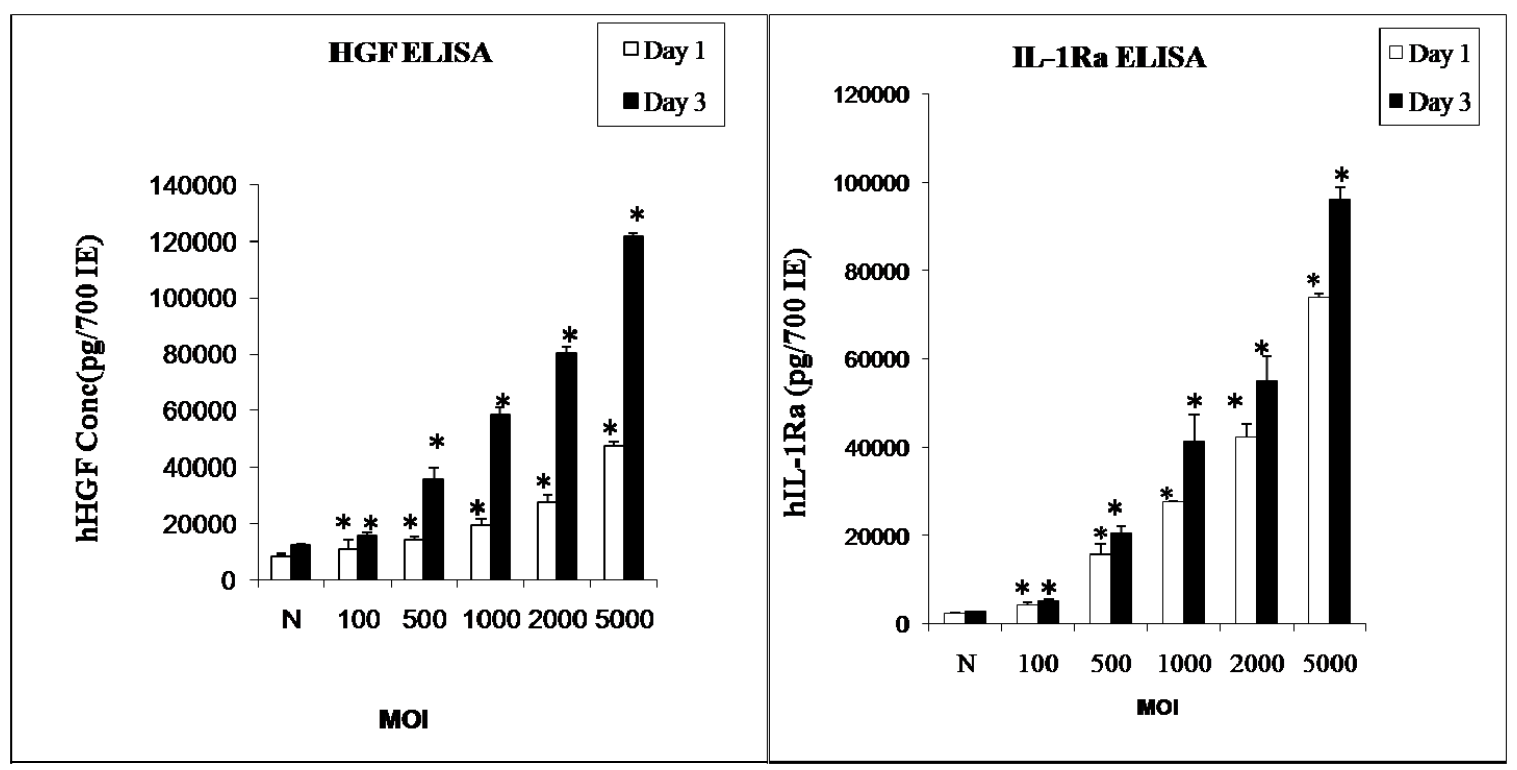

Figure 3-2 Expression of HGF and IL-1Ra genes at protein level.

Time profile of hHGF and hIL-1Ra gene expression at protein levels in human islets transduced Adv-HGF-IL-1Ra at different multiplicities of transduction (MOI) 100, 500, 1000, 2000 and 5000 per 1000 islets. Non-transduced islets were used as controls. ELISA of cell culture supernatants were performed at days 1 and 3. Cumulative expression levels were presented as the mean \pm S.D. $(n=4)$. N, non-transduced control islets. Difference between any two groups was determined by ANOVA. $\mathrm{P}<0.05$ was considered statistically significant. 
transduction would lead to high local concentrations of hHGF and hIL-1Ra in the immediate vicinity of islets post transplantation. We also determined hHGF and hIL-1Ra gene expression at mRNA levels by real time RT-PCR. As shown in Figure 3-3, the normalized mRNA concentration to that of the control was significantly increased with increase in MOI of Adv-hHGF-hIL-1Ra. Gene expression at mRNA level of hHGF and hIL-1Ra was more than 60 and 40 times higher than that of untransduced islets, respectively (Figure 3-3). Each graph is indicative of the relative increase in expression of hHGF and hIL-1Ra independently. This shows that Adv-hHGF-hIL-1Ra can simultaneously express both genes with high efficiency. The mRNA concentrations of each gene were normalized to that of the control i.e., untransduced islets. The amplification specificity was confirmed by melting peak analysis, which showed a single predominant peak each for $\mathrm{s} 19\left(84.5^{\circ} \mathrm{C}\right)$, hHGF $\left(78.8^{\circ} \mathrm{C}\right)$ and $\mathrm{hIL}-1 \mathrm{Ra}\left(85.5^{\circ} \mathrm{C}\right)$, respectively (data not shown).

\subsubsection{Effect of Viral Transduction on Islet Function}

To determine whether insulin secretion was adversely affected by transduction of islets with Adv-hHGF-hIL-1Ra, insulin secretion in response to glucose challenge was carried out at day 3 post-transduction at 500, 1000 and 2000 MOI, while non-transduced islets were used as the control. Glucose challenge was determined by quantifying glucose stimulated insulin release at basal level $(2.5 \mathrm{mM})$ and stimulated $(22 \mathrm{mM})$ glucose concentrations. There is a significant decrease in the stimulation index of un-transduced control islets when incubated with the cytokine cocktail compared to that in the absence of cytokine incubation (Figure 3-4).

Stimulation indices of Adv-hHGF-hIL-1Ra-transduced islets were almost identical to that of untransduced islets, when they were not incubated with the cytokine cocktail, suggesting that the transduction process does not cause any harm to the islets. Although incubation with the cytokine cocktail also led to decrease in the stimulation indices of Adv-hHGF-hIL-1Ra transduced islets, this decrease was much less compared to that of the un-transduced control islets, possibly due to the protective effect of hHGF and hIL1Ra co-expression (Figure 3-4).

\subsubsection{Apoptosis of Islets}

Apoptosis of islets leads to their impaired function and subsequent death, and thus it is an important detriment to islet transplantation. Caspase- 3 is the converging point of apoptotic pathways. Therefore, we determined the level of caspase- 3 in the islets transduced with Adv-hHGF-hIL-1Ra, following by incubation with the cytokine cocktail of IL-1 $\beta$, TNF- $\alpha$ and IFN- $\gamma$. Non-transduced control islets with and without cytokine treatment were used as controls. As shown in Figure 3-5, caspase-3 activity was high in the un-transduced islets treated with the cytokine cocktail compared to that in the absence of the cytokine cocktail. Transduction of islets with Adv-hHGF-hIL-1Ra protected islets from cytokine induced apoptosis as evidenced by low increase in caspase-3 levels 

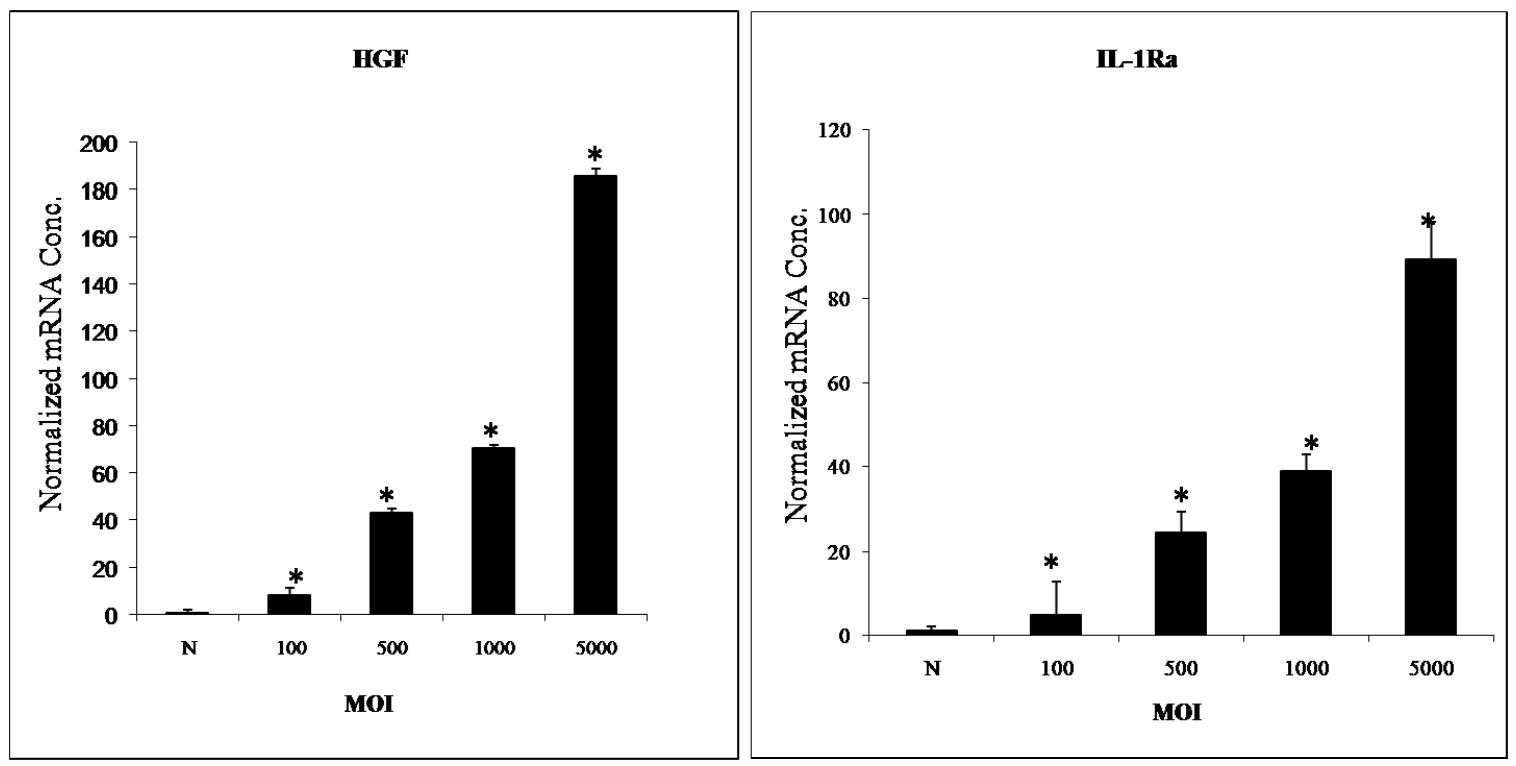

Figure 3-3 Real time PCR of Adv-hHGF-hIL-1Ra transduced human islets.

Real time PCR of human islets at day 3 post transduction with Adv-HGF-IL-1Ra at different MOI of 100, 500, 1000, 2000 and 5000. Forward and reverse primers were designed from hIL-1Ra and HGF genes. Non-transduced islets were used as controls. Cell pellet was collected at day 3 and processed for mRNA extraction, reverse transcription and real time PCR using SYBR green chemistry. The normalized mRNA values of the different MOI treated islets are plotted. Since different primers were used, no direct comparison can be made, other than demonstration of increase in mRNA levels with increase in MOI. N, non-transduced control islets. Difference between any two groups was determined by ANOVA. $\mathrm{P}<0.05$ was considered statistically significant. 


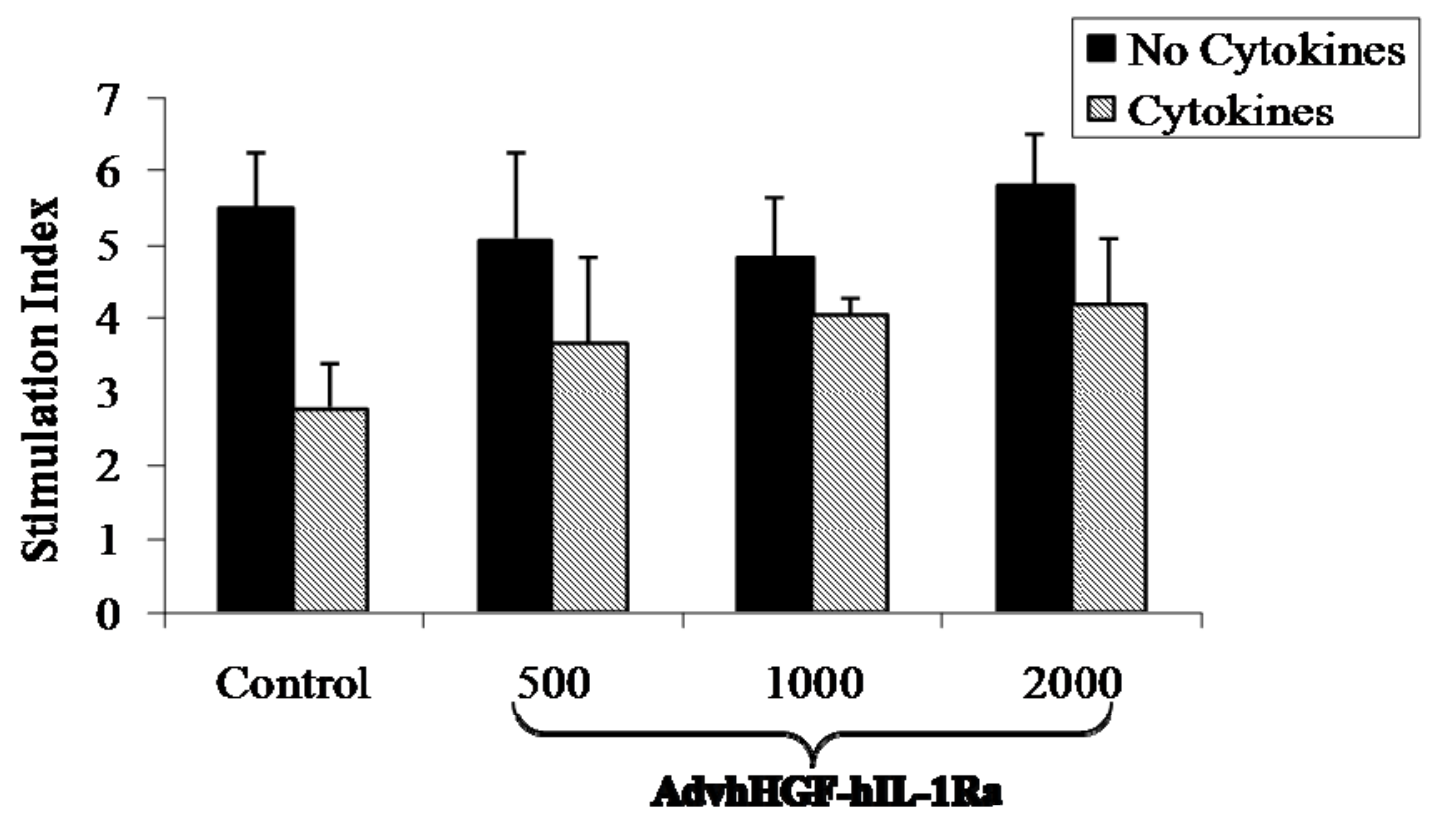

Figure 3-4 Glucose Stimulation Index.

Stimulation index (SI) of human islets with or without cytokine incubation after transduction with Adv-hHGF-IL-1Ra at 500, 1000 and 2000 MOI. Non-transduced islets were used as controls. SI was determined as the ratio of insulin release from islets when they are incubated at $396 \mathrm{mg} / \mathrm{dl}$ and $45 \mathrm{mg} / \mathrm{dl}$ glucose containing media. Increase in insulin secretion in response to high glucose concentration and decrease with reducing concentration is significant of metabolically healthy and functional islets. Results are expressed as a mean \pm S.D of $n=4$. 


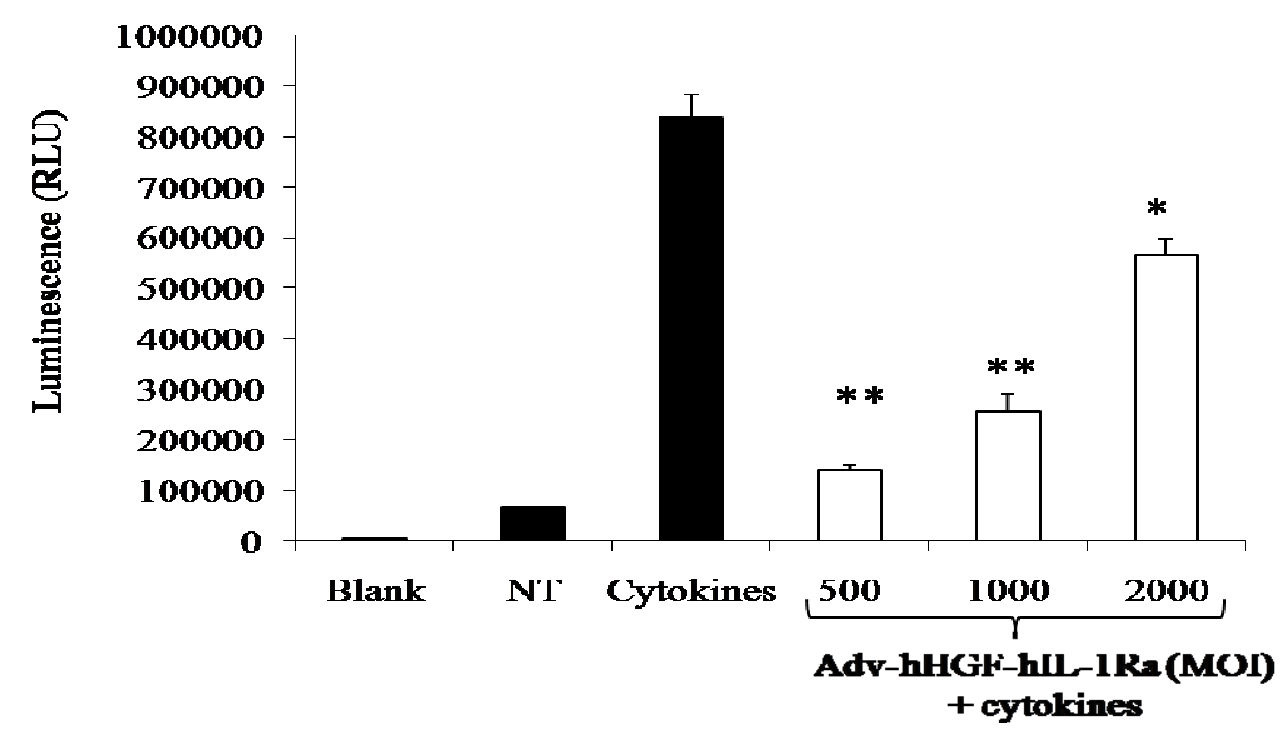

Figure 3-5 Effect of hHGF and hIL-1Ra co-expression on caspase-3 activity.

Following transduction with Adv-hHGF-hIlL-1Ra, islets were incubated with a cocktail of cytokines, such as IL-1 $\beta(10 \mathrm{ng} / \mathrm{ml})$, TNF- $\alpha(10 \mathrm{ng} / \mathrm{ml})$ and INF- $\gamma(50 \mathrm{ng} / \mathrm{ml})$. Non-transduced islets with and without cytokine treatment were used as controls. Blank media was also used as negative controls. Caspase-3 activity was determined at day 3 post-transduction by measuring the amount of caspase- 3 present in the lysate of islets after cell lysis using a Caspase-Glo 3/7 luciferase assay. Results are expressed as the mean \pm S.D. of $n=4$. ${ }^{*} \mathrm{p}<0.05$ and ${ }^{* *} \mathrm{p}<0.02$, compared to non-transduced islets with cytokine treatment. NT, non-transduced islets without cytokine treatment. 
compared to that of non-traduced islets incubated with the cytokines. Since the islets transduced at $2000 \mathrm{MOI}$ showed much higher level of caspase-3 activity compared to that of $1000 \mathrm{MOI}$ transduced islets, we decided to transduce islets at $1000 \mathrm{MOI}$ for in vivo testing. The inability of Ad5 vectors to be integrated into the host genome is another attribute of the Ad5 vectors that make them an attractive vector for transgene expression. Also the Ad5 vectors lacking the E1 and E3 genes render them to produce less immune response. ${ }^{170-172}$ The viral proteins synthesized will be minimal. However, a major concern in the use of adenoviral vectors is safety. Therefore, the vector associated immune response will be minimal. This is a very significant factor that needs to be thoroughly addressed before it can be used in a clinical setting. Although the adenoviral vectors are replication deficient, there are reports in the literature suggesting the immune response these vectors could elicit following their administration. ${ }^{85,172,173}$ We have seen that at higher doses 2000 MOI there was increase in caspase-3 activity in the islets transduced by the Adv indicating that higher doses of the Adv can be detrimental to the survival of islets following transplantation. This might be due to increase in vector backbone resulting in eliciting immune response. Also, the viral proteins can elicit an immune response. Therefore, we decided to use $1000 \mathrm{MOI}$ of Adv concentration in all the studies. We also determined the effect of Adv-hHGF-hIL-1Ra transduction on the levels of Bcl-2 (anti-apoptotic) and Bax (pro-apoptotic) by Western blot analysis. As shown in Figure 3-6, there was significant increase in Bcl-2 level, but decrease in Bax level.

\subsubsection{Islet Survival and Function after Transplantation}

The effect of hHGF and hIL-1Ra gene expression on the islet survival and function post-transplantation of 1000 islets transduced with Adv-hHGF-hIL-1Ra at $1000 \mathrm{MOI}$ under the kidney capsules of NOD-SCID mice was determined in terms of blood glucose, human insulin, and C-peptide levels. Following transplantation, blood glucose level decreased to around $200 \mathrm{mg} / \mathrm{dl}$ for both the un-transduced control and Adv-hHGF- hIL1Ra-transduced islet transplanted mice. However, the mice transplanted with untransduced islets showed gradual increase in the blood glucose level reaching to $300 \mathrm{mg} / \mathrm{dl}$ at day 28 post-transplantation. In contrast, no increase blood glucose level was seen in case of the mice transplanted with Adv-hHGF-hIL-1Ra-transduced islets even at 28 day post-transplantation (Figure 3-7). After removal of the kidney containing islets from some mice, blood glucose levels immediately returned to pre-transplant diabetic levels $(>500 \mathrm{mg} / \mathrm{dL})$, confirming that the transplant was responsible for reducing the blood glucose (Figure 3-7).To further define the function of the transplanted islets, glucose tolerance tests were performed at day 27 post-transplantation from the same mice.

After intraperitoneal glucose injection, blood glucose values were significantly lower in mice transplanted with Adv-hHGF-hIL-1Ra-transduced islets than in mice transplanted with uninfected islets (Figure 3-8). These results collectively indicate that at 4 weeks after transplantation of an equivalent mass of islets, Adv-hHGF-hIL-1Ra-transduced islets lead to superior glucose control and to tolerance as compared with control islets. 


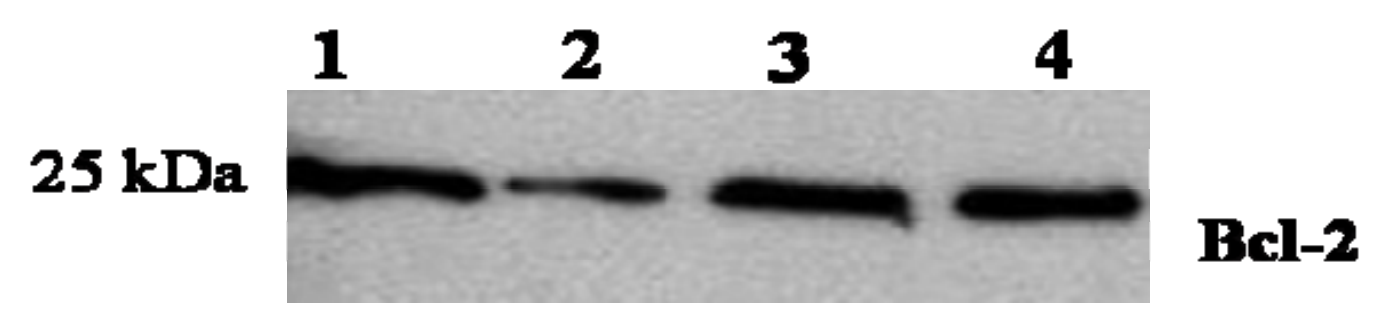

$21 \mathrm{kDa}$

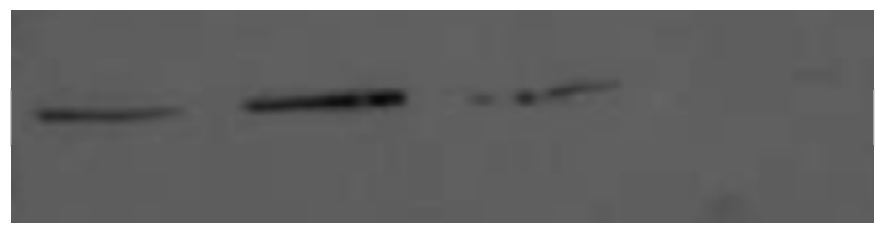

Bax

$43 \mathrm{kDa}$

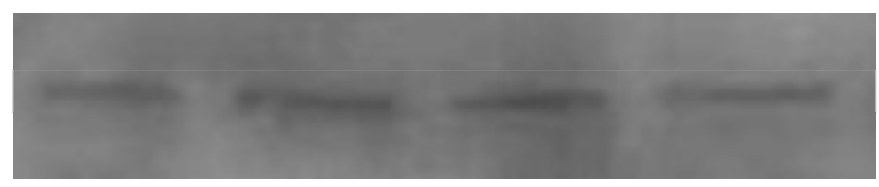

Actin

Figure 3-6 Effect of Adv-hHGF-hIL-1Ra on Bcl-2 and Bax protein levels.

Following transduction with Adv-hHGF-hIlL-1Ra, islets were incubated with a cocktail of cytokines, such as IL-1 $\beta(10 \mathrm{ng} / \mathrm{ml})$, TNF- $\alpha(10 \mathrm{ng} / \mathrm{ml})$ and INF- $\gamma(50 \mathrm{ng} / \mathrm{ml})$. Non-transduced islets with and without cytokine treatment were used as controls. Bcl-2 and Bax was determined at day 3 post-transduction by Western blot analysis. Lane 1, non-transduced islets; lane 2, non-transduced islets with cytokine treatment; lane 3, islets transduced with Adv-hHGF-hIL-1Ra at 500 MOI with cytokine treatment; and lane 4, islets transduced with Adv-hHGF-hIL-1Ra at 1000 MOI with cytokine treatment. 


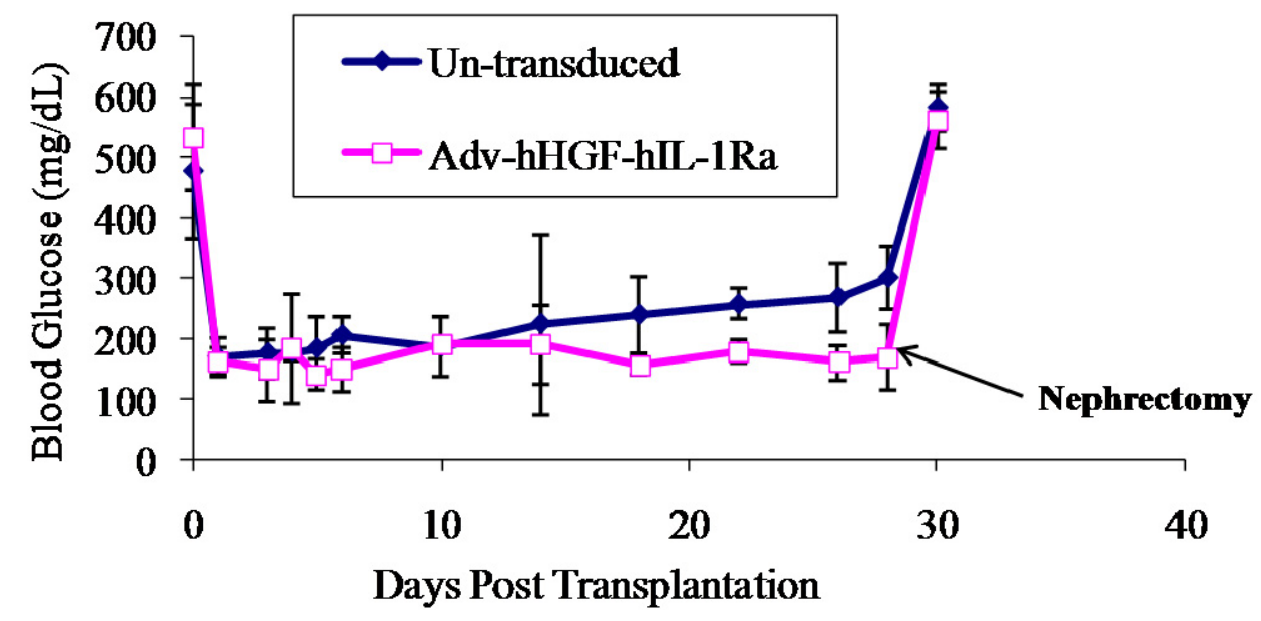

Figure 3-7 Transplantation of Adv-hHGF-hIL-1Ra transduced islets.

Following transduction with Adv-hHGF-hIL-1Ra at 1000 MOI, 1000 islets were transplanted under the kidney capsule of streptozotocin-induced diabetic NOD-SCID mice. The mice transplanted with un-transduced islets were used as controls. The nonfasting glucose levels were measured in each animal up to 28 days posttransplantation using a One Touch Ultra Glucometer (LifeScan, Inc.). 


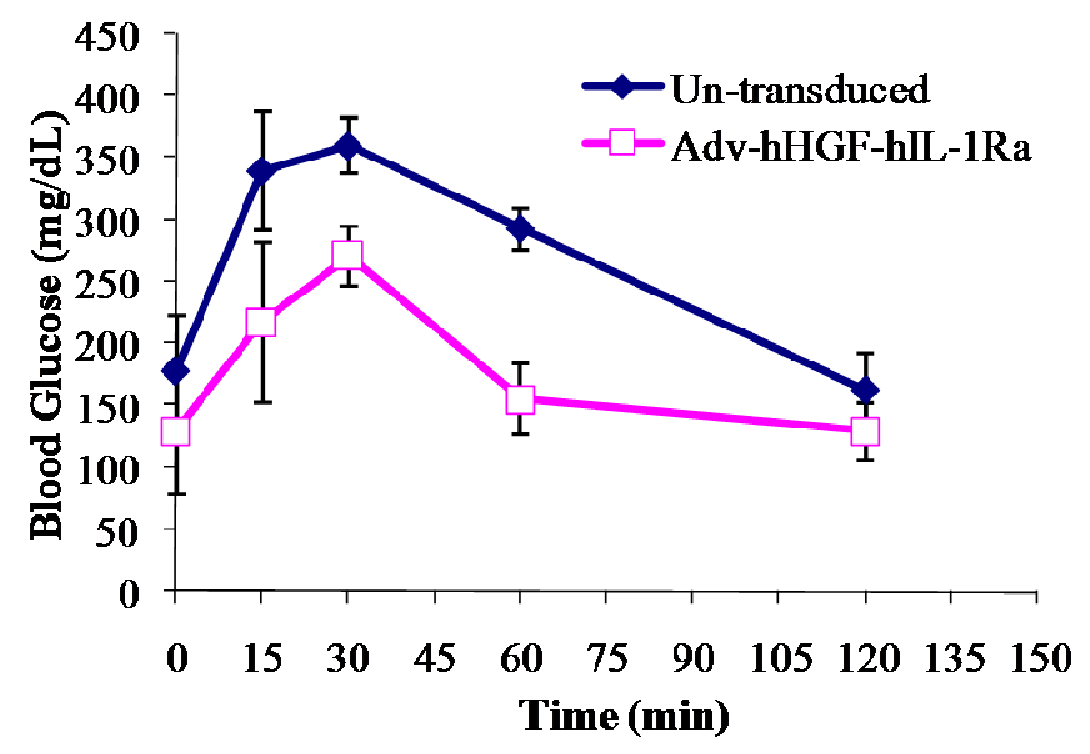

Figure 3-8 Intraperitoneal Glucose Tolerance Test.

Glucose tolerance in streptozotocin-induced NOD-SCID mice transplanted with uninfected or Adv-hHGF-hIL-1Ra transduced islets. At day 27 after the transplant, mice were fasted overnight and then injected intraperitoneally with glucose $(2 \mathrm{~g} / \mathrm{kg}$ of body weight). Blood glucose levels were measured from the snipped tail at the time points indicated with a glucometer. Results are the mean \pm S.D. of $n=4$. $* \mathrm{P}<0.05$ compared with control using ANOVA. 
Just before the removal of kidney containing islets, blood was collected for measuring the serum insulin and C-peptide levels. As shown in Figure 3-9A and B, serum levels of insulin and c-peptide were significantly higher in mice transplanted with Adv-hHGF-hIL1Ra transduced islets compared to un-transduced islet transplanted mouse groups, indicating better islet engraftment and function. These results suggest that compared to non-treated islets, co-expression of hHGF and hIL-1Ra sustained the low glucose level of mice for prolonged period, and increased the levels of serum insulin and c-peptide.

\subsubsection{Immunohistochemistry}

To determine whether hHGF gene expression after transduction with Adv-hVEGFhIL-1 Ra will promote angiogenesis by human islets, we isolated the kidney bearing AdvhHGF-hIL-1Ra transduced islets at 28 days post-transplantation into diabetic NOD-SCID mice for sectioning and staining with polyclonal rabbit hHGF and von Willebrand Factor (hvWF) antibodies. Staining with anti-human insulin and untransduced islet sections were used as the controls. The positive staining of hHGF was higher in the transduced islets compared to un-transduced islets (Figure 3-10), suggesting efficient hHGF gene expression after transduction with Adv-hHGF-hIL-1Ra. The positive staining of vWF factor was more evident in Adv-hHGF-hIL-1Ra transduced islets, which is an indicator for endothelial cells. Morphometric analysis revealed that Adv-hHGF-hIL-1Ra transduced islet grafts exhibited higher hHGF and vWF-immunostaining intensity than control islet grafts, with relative intensities of $1.70 \mathrm{vs}$. 1 for hHGF and $1.50 \mathrm{vs} .1$ for hvWF respectively.

To correlate the degree of islet revascularization with the islet mass in the kidney capsule, we also stained for human insulin. Adv-hHGF-hIL-1Ra transduced islets (Figure 3-10A) displayed significantly higher levels of insulin content than control islet groups (Figure 3-10D), with relative intensity of 1.7 vs. 1 . This quantitative difference in insulin content in the kidney capsule between Adv-hHGF-hIL-1Ra transduced and control islet groups of diabetic recipient mice correlated well with their serum insulin and c-peptide profiles (Figure 3-9). The positive staining for human insulin also confirmed the functionality of islets. These results indicate the beneficial effect of Adv-hHGF-hIL-1Ra for the islet survival and function post-transplantation.

\subsection{Discussion}

Isolation and purification of islets are known to disrupt their microvasculature, leading to hypoxia and apoptosis upon transplantation. ${ }^{133} \mathrm{HGF}$ has been shown to increase $\beta$-cell proliferation vitro and ameliorate hyperglycemia in streptozotocininduced diabetic mice transplanted with murine islet transplantation. ${ }^{164,165,174}$

Islet injury stimulates resident macrophages and infiltrating leukocytes to produce cytotoxic cytokines like IL-1 $\beta$, TNF- $\alpha$ and IFN- $\gamma$. Among these inflammatory cytokines, IL- $1 \beta$ is the key mediator of $\beta$ cell destruction. Administration of recombinant IL-1Ra 


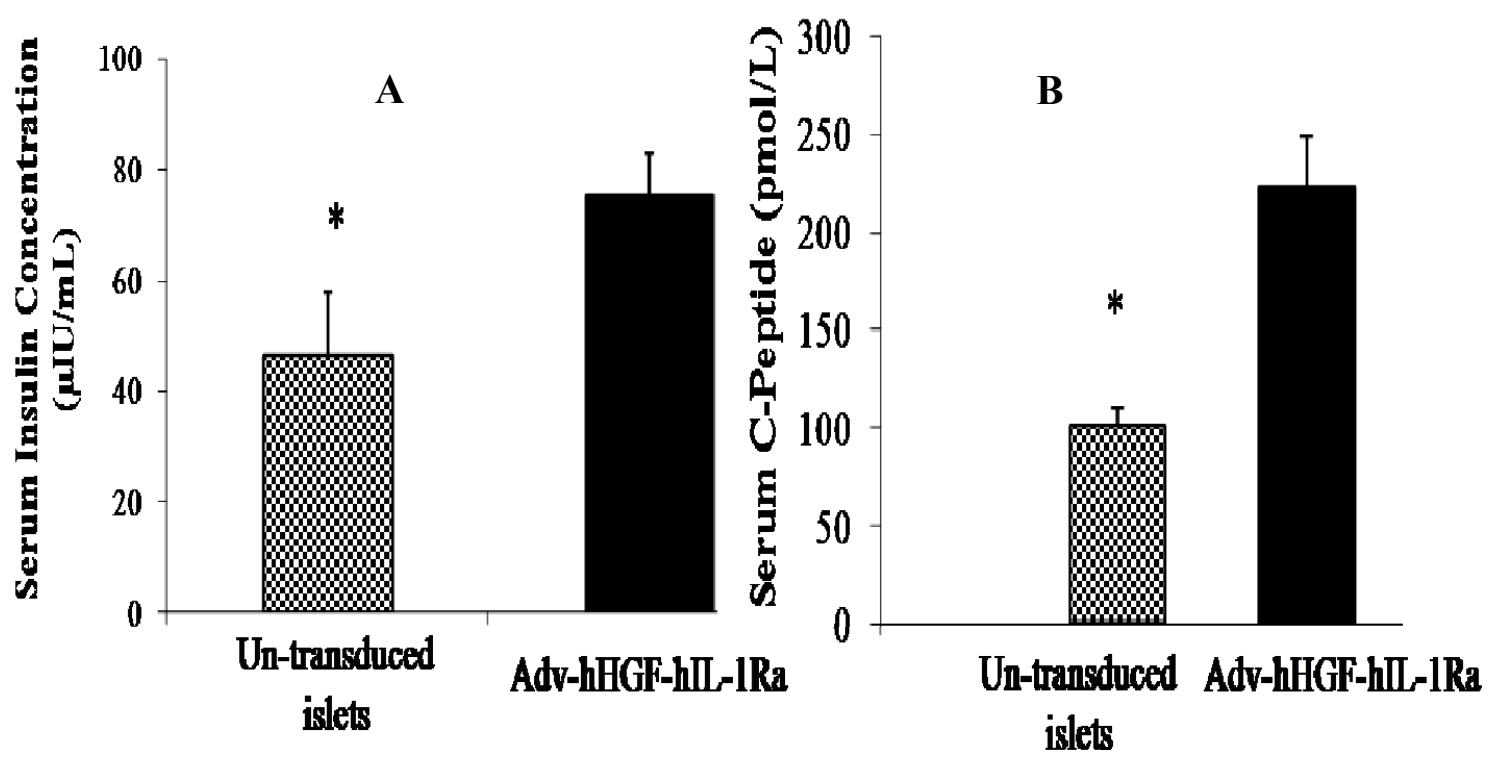

Figure 3-9 Serum insulin and c-peptide ELISA.

Following ex vivo transduction with Adv-hHGF-hIL-1Ra at 1000 MOI, 1000 islets were transplanted under the kidney capsule of streptozotocin-induced diabetic NODSCID mice. The mice transplanted with un-transduced islets were used as controls. At day 28 post-transplantation, the mice were sacrificed and blood was collected to measure A) serum insulin and B) C-peptide by ELISA. Results are expressed as the mean \pm S.D. $(\mathrm{n}=4) * \mathrm{p}<0.05$. 


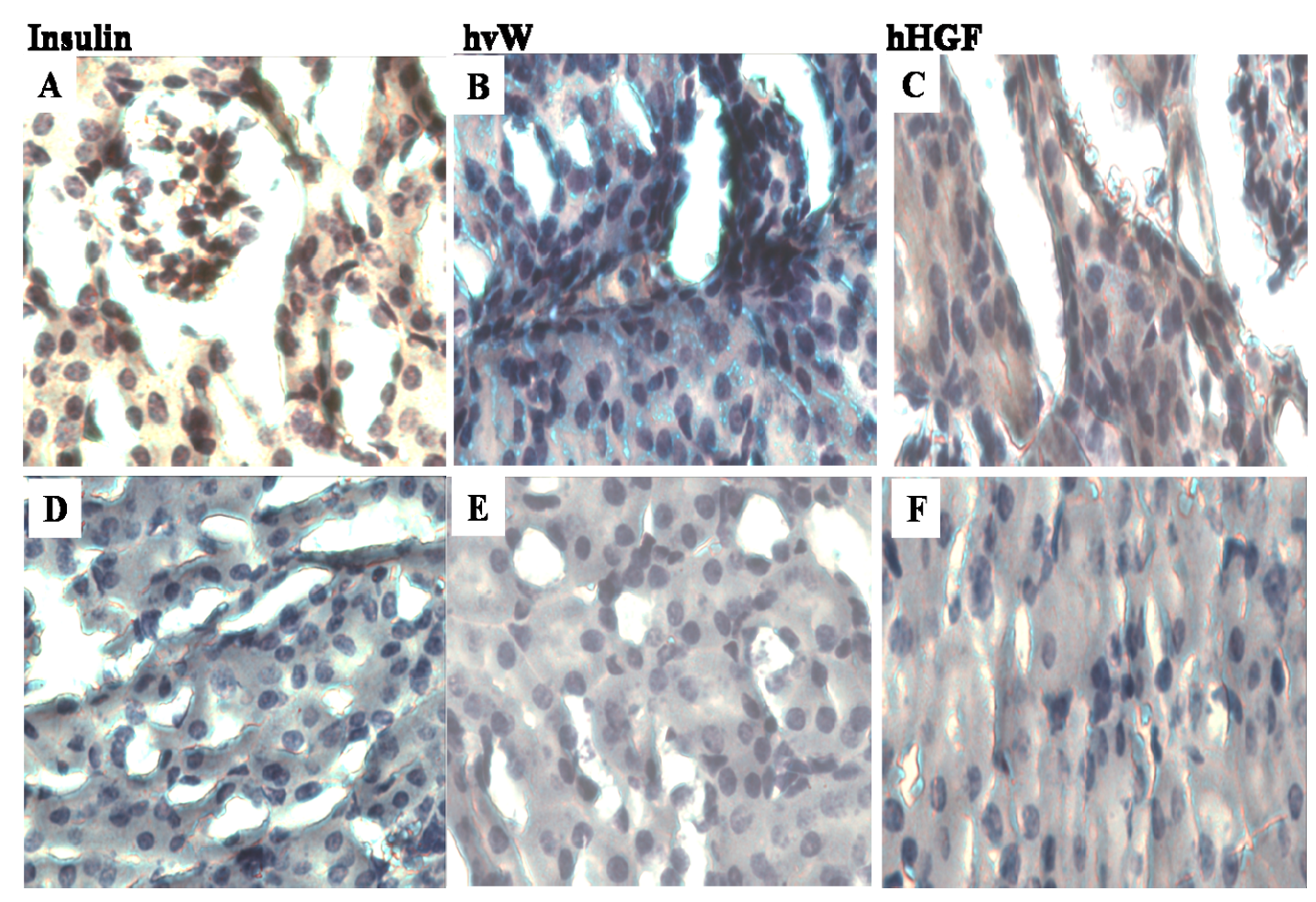

Figure 3-10 Immunohistochemical staining of islet bearing kidney sections.

Immunohistochemical staining of islet bearing kidney sections at 28 days posttransplantation of human islets transduced with Adv-hHGF-hIL-1Ra at $1000 \mathrm{MOI}$. Rabbit polyclonal anti-human HGF and hvW were used for detecting hHGF and endothelial cells. A) Insulin, B) hvW and C) hHGF staining for Adv-hHGF-hIL-1Ratransduced islets; and D) insulin, E) hvW and F) hHGF staining for the control islets. 
protein has been shown to prevent islet dysfunction upon transplantation. ${ }^{151}$ Ex vivo transduction of human islets with Adv-hIL-1Ra has been reported to prevent IL-1 $\beta$ induced $\beta$ cell impairment and activation of islet cell apoptosis. ${ }^{150}$ On the other hand, HGF is known to induce angiogenesis, regenerate $\beta$-cells and protect islets from apoptosis. Therefore, the objective of this study was to construct and characterize a bipartite Adv vector, Adv-hHGF-hIL-1Ra, for enhancing the performance of human islet grafts and correction of hyperglycemia upon transplantation under the kidney capsules of STZ-induced diabetic NOD-SCID mice.

Apoptosis begins during the islet isolation process, peaks 2-3 days post transplantation, and continues for approximately 7 14 days until what remains of the graft has stabilized and become vascularized. Therefore, the use of replication deficient Adv vectors promotes islet engraftment during the first week may be all that is necessary. The lack of integration is an advantage as well, since it will not allow induction of several key cell genes. The viral dose delivered to the patient by ex vivo gene therapy would be small reducing concerns regarding anaphylaxis. Finally, hHGF and hIL-1Ra coexpression will suppress immune reaction, leading to attenuation of host allergic reactions. Therefore, we decided to use $\Delta \mathrm{E} 1$ and $\Delta \mathrm{E} 3 \mathrm{Adv}$ vector because our goal was to achieve transient gene expression, while avoiding other potential side effects and in vivo virus replication. Since prolonged incubation with Adv vectors may induce apoptosis and loss of endothelial cells; we decided not to co-culture the islets beyond $12 \mathrm{~h}$, we determined HGF and hIL-1Ra gene expression at protein and mRNA levels. The protein and mRNA levels of HGF and hIL-1Ra increased with increase in MOI (Figures 3-2 and 3-3), indicating the Adv vector is indeed expressing these two genes as desired. However, these results cannot be directly correlated, since we used different primers for different genes and thus we can only compare our real time RT-PCR results in relative to the house keeping genes.

Apoptosis is a major pathway through which islets undergo cell death and has been considered as a major factor for primary graft non-function. ${ }^{175}$ To mimic the in vivo situation of the cytokine mediated insults, we incubated islets with a cocktail of inflammatory cytokines such as IL- $1 \beta$, TNF- $\alpha$ and IFN- $\gamma$. Caspase- 3 is a converging point of apoptosis for intrinsic and extrinsic pathways and caspase- 6 and 7 are generated from caspase-3. There was gradual increase in caspase- 3 with increase in Adv-hHGF$\mathrm{hIL}-1 \mathrm{Ra}$ in the presence of these cytokines, but caspase- 3 levels were much lower compared to the un-transduced islets (Figure 3-5). The levels of caspase-3 at 500 and $1000 \mathrm{MOI}$ was low, suggesting these Adv vectors are fairly safe at these tested doses. Bcl-2 family of intracellular proteins plays an important role in regulation of cytochrome $\mathrm{C}$ release into the cytosol and caspase activation. Anti-apoptotic members, such as Bcl-2, $\mathrm{Bcl}-\mathrm{X}_{\mathrm{L}}$ and Bcl-w are critical for cell survival, whereas pro-apoptotic members, such as Bax, Bad and Bak among others promote apoptosis. ${ }^{176}$ Heterodimerization of Bcl-2 with Bax or Bak modulates apoptosis, and the ratio of Bcl-2 to Bax or Bak determines survival or death following an apoptotic stimulus. Transduction of islets with Adv-hHGF-hIL-1Ra greatly enhanced the level of Bcl-2 protein while inhibited Bax protein level (Figure 3-6). This result further demonstrates the protective effect of hHGF and hIL-1Ra co-expression since $\mathrm{Bcl}-2$ has been shown to inhibit cytochrome $\mathrm{C}$ release and protect against oxidative 
stress-induced apoptosis, ${ }^{177}$ indicating the usefulness of this vector in protecting islets from apoptosis following transplantation.

Effect of Adv-hHGF-hIL-1Ra transduction on improvement in the survival and function of islets post-transplantation was determined in streptozotocin-induced diabetic NOD-SCID mice. We observed a reduction in blood glucose levels of mice transplanted with Adv-HGF-hIL-1Ra transduced islets than that observed with un-transduced islets, especially after 5 days post-transplantation, but increase in blood glucose levels for the mice transplanted with un-transduced islets (Figure 3-6). This decrease in blood glucose levels by Adv-hHGF-hIL-1Ra-transduced mice correlated with the higher amount of serum insulin and C-peptide secreted by the islets at day 28 post-transplantation (Figure 3-9A and B). Our results are in good agreement with Lopez-Talavera et al., ${ }^{146}$ who observed glycemic control in Adv-HGF-transduced islet transplanted rats superior to the tat observed in diabetic control rats. Revascularization of islet grafts after transplantation is an important process that influences the long-term survival and function of the grafts. Endothelial cells reside within isolated islets and potentially participate in the revascularization process. Immunohistochemical staining in the islet bearing kidney sections at day 28 after transplantation was positive for human insulin, hHGF and vWF (Figure 3-10). Positive staining for insulin, which stains pancreatic $\beta$-cells corroborates the serum insulin data (Figure 3-9A) and confirms that islets are indeed functional after transplantation, which is in good agreement with the contemporary literature. vWF is human endothelial cell marker. Therefore, relatively stronger positive staining for hHGF and vWF antigens by the Adv-hHGF-hIL-1Ra transduced islet bearing kidney section groups indicates that blood vessel formation in the transplanted islets was relatively more efficient when islets were transduced with Adv-hHGF-hIL-1Ra prior to transplantation.

These results indicate that HGF and hIL-1Ra co-expression is likely to reduce the islet mass required to achieve normoglycemia. Although we did not examine the apoptosis in graft islet cells, our in vitro data clearly indicated that transduction of AdvHGF-hIL-1Ra led to significant reduction of caspase-3 activity, which is the indicator of apoptosis.

We have tested two different adenoviral vectors Adv-hVEGF-hIL-1Ra and AdvhHGF-hIL-1Ra by transducing them into human islets and studying their survival and function following transplantation in NOD-SCID mice. Both VEGF and HGF are angiogenic growth factors. However, we replaced VEGF with HGF as HGF not only promotes revascularization, but also can protect the islets from apoptosis. It can also promote the proliferation of $\beta$-cells and also has shown to decrease the islet mass needed for achieving normoglycemia. In our studies, we have seen that the islets transduced with Adv-hHGF-hIL-1Ra maintained normoglycemia in diabetic NOD-SCID mice for longer period compared to islets transduced with Adv-hVEGF-hIL-1Ra. Also, we have seen the toxicity of adenoviral vectors at higher doses thereby needing to decrease the viral dose for transducing islets prior to transplantation. Considering all these factors, it can be clearly said that Adv-hHGF-hIL-1Ra is more apt for transducing the human islets to improve their survival and function following transplantation. Although, further studies are warranted to study the immunogenicity of the adenoviral vectors, their effect 
following transplantation of Adv transduced islets in the hepatic portal vein mimicking the human setting to clearly say about the usefulness of these vectors in clinical setting. 


\section{CHAPTER 4. TRIPLEX FORMING OLIGONUCLEOTIDES AGAINST TYPE ALPHA 1(I) COLLAGEN ATTENUATES LIVER FIBROSIS INDUCED BY BILE DUCT LIGATION}

\subsection{Introduction}

Liver fibrosis results from chronic damage to the liver in conjunction with the accumulation of extracellular matrix (ECM) proteins, which is a characteristic of most chronic liver diseases. ${ }^{68}$ The main causes of liver fibrosis in industrialized countries include chronic hepatitis $\mathrm{C}$ virus (HCV) infection, alcohol abuse, and nonalcoholic steatohepatitis (NASH). Such chronic diseases lead to scarring in the liver that, with increasing severity, prevents effective liver regeneration and maintenance of liver function. ${ }^{178}$ Liver fibrosis is associated with major alterations in both the quantity and composition of ECM. The accumulation of ECM proteins distorts the hepatic architecture by forming a fibrous scar, and the subsequent development of nodules of regenerating hepatocytes defines cirrhosis. ${ }^{179}$

Type I collagen, the major component of the extracellular matrix in the fibrotic liver, is a heterotrimer composed of $2 \alpha 1$ chains and $1 \alpha 2$ chain. These chains are encoded by 2 distinct genes, COLlAl and COLlA2, respectively. Hepatic stellate cells (HSCs; also known as vitamin A-storing cells, fat-storing cells, Ito cells, and lipocytes) residing in the perisinusoidal space of Disse in the liver, are the main producers of type $\alpha 1$ (I) collagen and other components of extracellular matrix in both normal and fibrotic livers. ${ }^{180,181}$ Following chronic injury, cytokines such as transforming growth factor $\beta$ (TGF- $\beta$ ) and protein kinase signaling pathways mediate a complex interplay among different hepatic cells. ${ }^{182-184}$ Under the influence of these signals, HSCs activate or transdifferentiate into myofibroblast-like cells, acquiring contractile, proinflammatory, and fibrogenic properties resulting in increased synthesis of types I and III fibrillar collagen. ${ }^{185,186}$

In contrast to the traditional view that cirrhosis is an irreversible disease, recent evidence indicates that even advanced fibrosis is reversible. ${ }^{187,188}$ Preclinical studies have reported a scientific rationale and experimental evidence supporting the use of many potential therapies for fibrosis. Such therapies have been targeted to any of several different biological targets (e.g., inhibition of collagen synthesis, interruption of matrix deposition, stimulation of matrix degradation, modulation of stellate cell activation, or induction of HSC death). Triplex-forming oligonucleotides (TFOs) constitute an interesting DNA sequence-specific tool that can be used to target cleaving or crosslinking agents, transcription factors or nucleases to a chosen site on the DNA. TFOs have proved effective in altering gene expression by interfering with the binding of transcription factors. ${ }^{189,190}$ Because liver fibrosis is due to the overproduction of Type I collagen by liver fibrogenic cells, targeted inhibition of transcription of type $\alpha 1$ (I) collagen gene is expected to prevent fibrosis. Recently, we showed that antiparallel phosphorothioate (APS) TFOs specific for $\alpha 1$ (1) collagen form triplexes efficiently and inhibit transcription in cultured immortalized rat hepatic stellate cells (HSC-T6) in 
vitro. ${ }^{191}$ These results suggest that targeting collagen transcription may theoretically attenuate fibrosis in livers undergoing tissue remodeling.

In the current study, we studied the antifibrotic effects of TFOs in rats undergoing common bile duct ligation (CBDL). TFO administration in CBDL rats resulted in decreased liver injury, inflammation, and fibrosis. At the cellular level, TFO induced similar effects in primary rat HSCs. This evidence indicates that the prevention of fibrosis by anti-collagen TFO treatment could preserve organ function and be therapeutically useful. Our data may suggest that anticollagen TFOs could be effective in future gene therapy for fibrosis in vital organs.

\subsection{Materials and Methods}

\subsubsection{Materials}

TFO, which was a 25-mer antiparallel fully phosphorothioate ODN (3'GAGGGGGGAGGAGGGAAAGGAAGGG-5') targeting rat $\alpha 1$ (I) collagen gene promoter was synthesized by Invitrogen (Carlsbad, CA). Bovine serum albumin (BSA) (fraction V, purity $>98 \%$ ) was purchased from USB Corporation (Cleveland, OH). SYBR Green-1 dye universal master mix and MultiScribe reverse transcriptase were purchased from Applied Biosystems, Inc. (Foster City, CA). Tumor necrosis factor (TNF)- $\alpha$ ELISA kits were purchased from eBioscience, Inc. (San Diego, CA). RNA extraction kit was procured from Promega (Madison, WI). Immobilon polyvinylidene fluoride (PVDF) membrane was purchased from Millipore (Billerica, MA). Rabbit antirat TGF- $\beta 1$ and $\beta$ actin primary antibodies and horseradish peroxidase-conjugated goat antirabbit secondary antibody were purchased from Santa Cruz Biotechnology (Santa Cruz, CA). Chemiluminescence (ECL) detection kit was purchased from GE Healthcare Life Sciences (Pittsburgh, PA). Serum alanine transaminase and aspartate transaminase kits were purchased from ID Labs, Inc (London, Canada). Citric acid and sodium citrate were procured from Curtin Matheson Scientific, Inc (Houston, TX). Sodium hydroxide was purchased from Fisher Scientific, (Fair Lawn, NJ). Hydroxyproline, Chloramine-T, 3, 3'diaminobenzidine (DAB) and goat serum were purchased from Sigma-Aldrich (St. Louis, $\mathrm{MO})$.

\subsubsection{Animals and Experimental Design}

Male Sprague-Dawley rats weighing 250-280g were maintained under conditions as per the NIH (http:/grants1.nih.gov/grants/olaw/references/phspol.htm) and institutional animal care and use Committee using the approved protocol. All animals were housed in microisolator cages in virus-free facilities and fed laboratory chow ad libitum. CBDL or

sham operation was performed as described previously. ${ }^{192-194}$ After a midline laparotomy, liver lobes were retracted upwards and the intestines were reflected to the right to expose the common bile duct (CBD). The CBD was then isolated by careful blunt dissection, 
doubly ligated with 4-0 silk and transected between the two ligations. The sham operation was performed similarly, with the exception of ligating and transecting the bile duct.

Liver was then replaced into the abdominal cavity making sure there were no twists in the vascular pedicles of its lobes. The abdomen was closed with 4-0 silk running suture. Rats were injected with TFO at $8 \mathrm{mg} / \mathrm{kg}$ per dose twice a week for 4 weeks via the tail vein. Sham operated rats and CBDL control rats were injected with an equal volume of saline. Animals were sacrificed after 7 weeks. Blood and liver tissue were collected for biochemical, RNA, histological and immunohistochemical studies.

\subsubsection{Cell Culture}

Immortalized rat hepatic stellate cells (HSC-T6) were a kind gift by Dr. Scott Friedman (Mount Sinai School of Medicine, New York). HSC-T6 cells at a density of $1 \mathrm{X} 10^{6}$ cells were seeded on uncoated plastic tissue culture 6-well plates and cultured in Dulbecco's Modified Eagle Medium (DMEM, Gibco BRL, Grand Island, NY) containing $10 \%$ of fetal bovine serum (FBS) until $50 \%$ confluence was achieved. The growth medium was replaced with a pre-warmed serum-free DMEM. TFO was mixed with lipofectamine 2000 according to the manufacture's protocol and then used for transfection. Scrambled TFO was used as control.

\subsubsection{Serum Transaminase Levels}

Serum levels of aspartate aminotransferase (AST) and alanine aminotransferase (ALT) were used as markers of liver injury. ALT and AST concentrations were measured using IDTox ${ }^{\mathrm{TM}}$ Alanine transaminase color endpoint assay kit and IDTox ${ }^{\mathrm{TM}}$ Aspartate transaminase enzyme assay kit (ID Labs ${ }^{\mathrm{TM}}$ Inc, London, ON, Canada) according to the manufacturer's instructions and absorbance was measured using a spectrophotometer.

\subsubsection{Hydroxyproline Assay}

Hydroxyproline content was quantified colorimetrically from 30mg of liver tissue as previously described. ${ }^{195}$ Briefly, fresh liver tissue was collected and weighed at the time of sacrificing the animals. The tissue was then hydrolyzed with $6 \mathrm{~N} \mathrm{HCl}$ and then heated in an oven at $110^{\circ} \mathrm{C}$ for $18 \mathrm{~h}$. The tissue was incubated in a vacuum desiccator overnight until the $\mathrm{HCl}$ was completely dried. Citrate buffer was added to dilute the samples. Then, Chloramine-T reagent was added to the samples and allowed to react for $20 \mathrm{~min}$ at room temperature. Following this, fresh Ehrlich's reagent was prepared and added to each sample. Samples were then placed in a warm water bath $\left(60^{\circ} \mathrm{C}\right)$ and allowed to react for $15 \mathrm{~min}$. Samples were then cooled to room temperature and absorbance was read at $550 \mathrm{~nm}$ for each sample and a standard control. The results were expressed as micrograms of hydroxyproline per grams of liver. Hydroxyproline standard curve was also prepared using variable concentrations of it and plotting the standard curve after measuring the 
absorbance at 560nm. Hydroxyproline levels correspond to the total amount of collagen present.

\subsubsection{ELISA for Tumor Necrosis Factor- $\alpha$}

Cell culture supernatant or serum was used to determine the levels of TNF- $\alpha$ using an ELISA kit according to the manufacturer's specifications (eBiosciences, San Diego, CA). For in vitro studies, rat HSC-T6 cells were cultured as described above. Medium was removed and cells incubated in serum-free medium for $24 \mathrm{~h}$. Supernatants were collected and stored at $-80^{\circ} \mathrm{C}$ until analysis. For in vivo studies, blood was collected immediately after euthanasia and centrifuged at $10,000 \mathrm{rpm}$ for $7 \mathrm{~min}$ at room temperature to separate the serum. Serum was at $-80^{\circ} \mathrm{C}$ for future use. ELISA data were then presented as the percentage $(\%)$ of the control for in vitro studies or picograms per milliliter of serum for in vivo studies.

\subsubsection{Myeloperoxidase (MPO) Activity Assay}

The presence of MPO, an enzyme specific for neutrophils, was used as an index of liver neutrophil accumulation. Briefly, the frozen tissue was thawed and placed in $4 \mathrm{ml}$ iced $0.5 \%$ hexadecyltrimethylammonium bromide and $50 \mathrm{mmol}$ potassium phosphate buffer solution with the $\mathrm{pH}$ adjusted to 5 . Each sample was homogenized for $30 \mathrm{~s}$ and centrifuged at $15,000 \mathrm{rpm}$ for $15 \mathrm{~min}$ at $4^{\circ} \mathrm{C}$. Supernatants were then mixed with hydrogen peroxide-sodium acetate and tetramethyl benzidine solutions. The change in absorbance was measured spectrophotometrically at $460 \mathrm{~nm}$. One unit of MPO activity was defined as the quantity of enzyme degrading 1 mol peroxide per minute at $25^{\circ} \mathrm{C}$ per gram of tissue.

\subsubsection{Real Time Polymerase Chain Reaction}

To determine Type I collagen, alpha smooth muscle actin ( $\alpha$-SMA), TGF- $\beta 1$, and tissue inhibitor of metalloproteinase 1 (TIMP-1) gene expression in rat liver, total liver RNA was extracted using RNeasy extraction kit (Qiagen, Valencia, CA). RNA concentration and purity was measured and confirmed using Nanodrop (Thermo Scientific, Wilmington, DE). Total RNA (385ng) was reverse transcribed to cDNA templates using MultiScribe reverse transcriptase and random hexamers by incubation at $25^{\circ} \mathrm{C}$ for $10 \mathrm{~min}$, followed by reverse transcription at $48^{\circ} \mathrm{C}$ for $30 \mathrm{~min}$ and enzyme inactivation at $95^{\circ} \mathrm{C}$ for $5 \mathrm{~min}$. In all 100ng of cDNA was amplified by real time PCR using SYBR Green dye universal master mix on an LightCycler ${ }^{\circledR} 480$ (LC 480) (Applied Biosystems, Inc., Foster City, CA) using the primers (Table 4-1) for Type I collagen (NCBI Accession\#NM_053304.1), $\alpha$-SMA (NCBI Accession\#NM_031004.2), TGF$\beta 1(N C B I$ Accession\#NM_021578.2), TIMP-1(NCBI Accession\#NM_053819.1). The PCR products were subjected to a melting curve analysis and crossing point $(\mathrm{Cp})$ was used for calculating the relative amount of mRNA compared to the house keeping gene, 
Table 4-1 Primers for real time PCR.

Gene Forward Primer (5'-3') Reverse Primer (5'-3')

$\alpha 1(\mathrm{I})$

collagen TGGTCCCAAAGGTTCTCCTGGT TTAGGTCCAGGGAATCCCATCACA

$\alpha$-SMA ACAACGTGCCTATCTATGAGGGCT AGCGACATAGCACAGCTTCTCCTT

TGF- $\beta 1$ CATCCATGACATGAACCGACCCTT ACAGAAGTTGGCATGGTAGCCCTT

TIMP-1 CCTCTGGCATCCTCTTGTTGCTAT CATTTCCCACAGCGTCGAATCCTT 
hypoxanthinephophoribosyltransferase (HPRT), and then scaled relative to controls, where control samples were set at a value of 1 . Thus, results for all experimental samples were graphed as relative expression compared with the control. Melting curve analysis was also done to check the amplification specificity.

\subsubsection{Histological Staining}

Formalin-fixed liver specimens from the TFO treated, BDL and Sham operated rats were dehydrated in alcohols, incubated in xylene, and embedded in paraffin. Fivemicron-thick tissue sections were cut and stained with either hematoxylin-eosin for general histology or Masson's trichrome for collagen staining, according to manufacturer's protocols.

\subsubsection{Immunofluorescent Staining}

Immunofluorescent staining was performed on snap frozen liver tissue. Briefly, $5 \mu \mathrm{m}$ cryosections were cut on lysine coated slides and fixed in $95 \%$ cold ethanol. Slides were air dried and stored at $-80^{\circ} \mathrm{C}$ till further use. The sections were blocked with $10 \%$ goat serum with $1 \% \mathrm{BSA}$ in PBS for $2 \mathrm{~h}$ at room temperature. Cryosections then incubated with the following primary antibodies overnight at $4^{\circ} \mathrm{C}$ : anti rabbit $\alpha$ - SMA, anti rabbit TGF- $\beta$, and anti-mouse cytoketain- 19 . The following secondary antibodies were used, anti-rabbit Alexa Fluor 488, anti-mouse Alexa Fluor 594 and anti-rabbit Alexa Fluor 594. Nuclear staining was performed using 4', 6-diamidino-2-phenylindole (DAPI). Immunofluorescence was visualized on a Zeiss Apoplan Microscopy system.

\subsubsection{Statistical Analysis}

Data are expressed as the mean \pm standard deviation (SD). Difference between any two groups was determined by ANOVA and t-test. $\mathrm{P}<0.05$ was considered statistically significant.

\subsection{Results}

\subsubsection{TFO Downregulates Type 1 (I) $\alpha$-Collagen In Vitro}

HSCs were cultutred and transfected with $1 \mu \mathrm{g}$ of TFO. mRNA analysis showed a drastic decrease in the expression of type 1 (I) $\alpha$-Collagen in the HSCs transfected with TFO inhibiting type 1 (I) $\alpha$-Collagen compared to that of scrambled TFO and untransfected HSCs (Figure 4-1). 


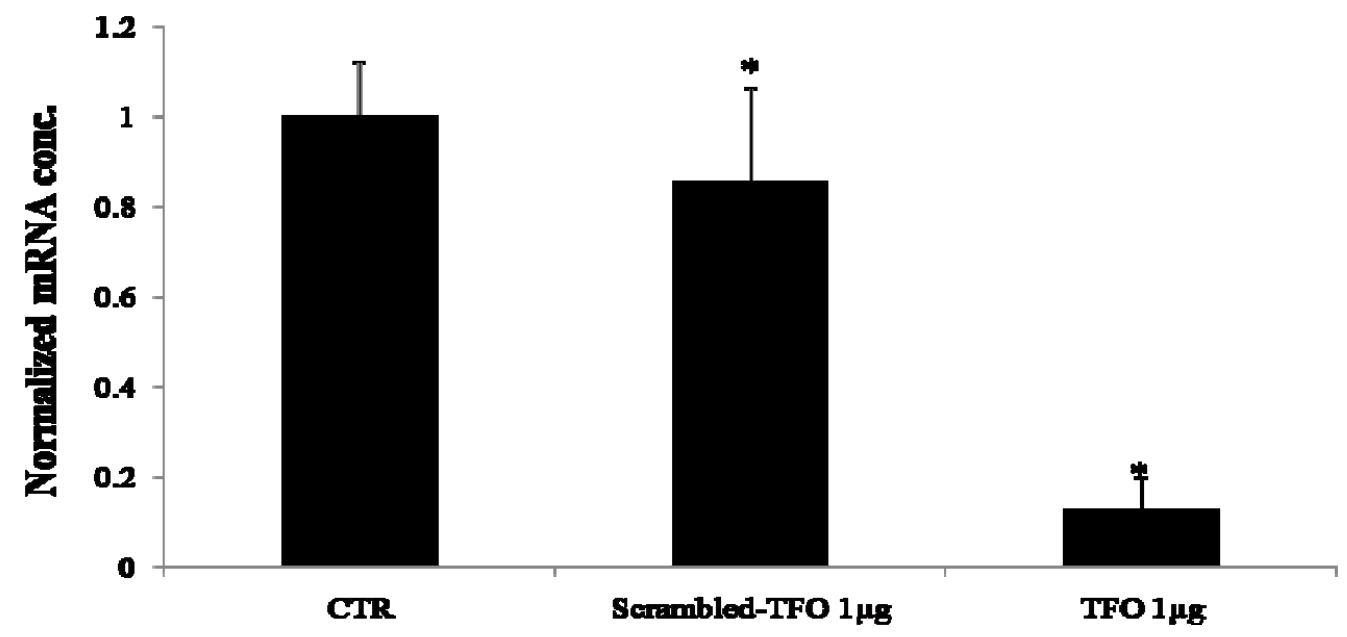

Figure 4-1 TFO down regulated type 1 (I) $\alpha$-collagen in HSCs. 
4.3.2. TFO Downregulates Profibrogenic and Proinflammatory Cytokines in HSCs In Vitro

TGF- $\beta 1$ and TIMP- 1 are the two major profibrogenic cytokines implicated in the development of liver fibrosis. ${ }^{196-198}$ As shown in Figure 4-2A, transfection of HSC-T6 cells with $1 \mu \mathrm{g}$ of TFO complexed with Lipofectamine significantly attenuated expression of TIMP 1 and TGF- $\beta 1$ mRNAs on real time PCR. Similarly, TFO significantly decreased proinflammatory cytokine TNF- $\alpha$ concentration in cultured medium as measured by ELISA (Figure 4-2B).

\subsubsection{TFO Attenuates Chronic Liver Injury in CBDL Rats}

To examine the effect of TFO on liver fibrosis, the common bile ducts of rats were ligated and at 3 weeks post CBDL, three rat livers were isolated to confirm liver fibrosis by evaluating i) macroscopically, ii) hydroxyproline assay and Masson's trichrome staining (data not shown). Then, TFO was injected intravenously twice a week at a dose of $8 \mathrm{mg} / \mathrm{kg}$ for 4 consecutive weeks and the degree of liver fibrosis was assessed by using three independent methods: collagen quantitation by measuring hydroxyproline content, Col 1A2 mRNA level in the liver samples and histological analysis by hematoxylin and eosin histological staining as well as Masson's trichrome staining. Hydroxyproline is a quantitative measure of collagen deposition and fibrosis. ${ }^{199}$ As expected, there was significant increase in hydroxyproline content in the liver following CBDL, indicating that the liver fibrosis model was successfully established. TFO treatment reduced hydroxyproline content in the fibrotic liver, which was similar to that of the normal rats (Figure 4-3).

To determine whether the decrease in hydroxyproline content in the liver of TFO treated CBDL rats was due to the transcription inhibition of $\alpha 1$ (1) collagen, we determined collagen mRNA levels by real time PCR. The levels of $\alpha 1$ (I) collagen mRNA (corrected by the steady-state levels of HPRT mRNA) were decreased in the liver of TFO treated CBDL rats.

\subsubsection{Histopathological Alterations}

Liver architecture of fibrotic livers with or without TFO treatment was determined by hematoxylin and eosin histological staining. As shown in Figure 4-4, there was significant number of bile infarcts noticed in the BDL liver sections. TFO treatment showed significant reduction in the bile infarcts. Figure 4-5 shows Masson's trichrome staining of the liver sections of the control, CBDL and TFO-treated rat livers. In the control rats which were exposed to saline only, deposition of collagen was found surrounding the central veins and enclosing portal triads representing the collagen distribution of healthy livers. CBDL rats being treated with saline over 4 weeks displayed a periportal fibrosis characterized by portal-portal septa surrounding the lobules. Collagen deposition was extensive in the peribiliary and interstitial tissue. There were also 

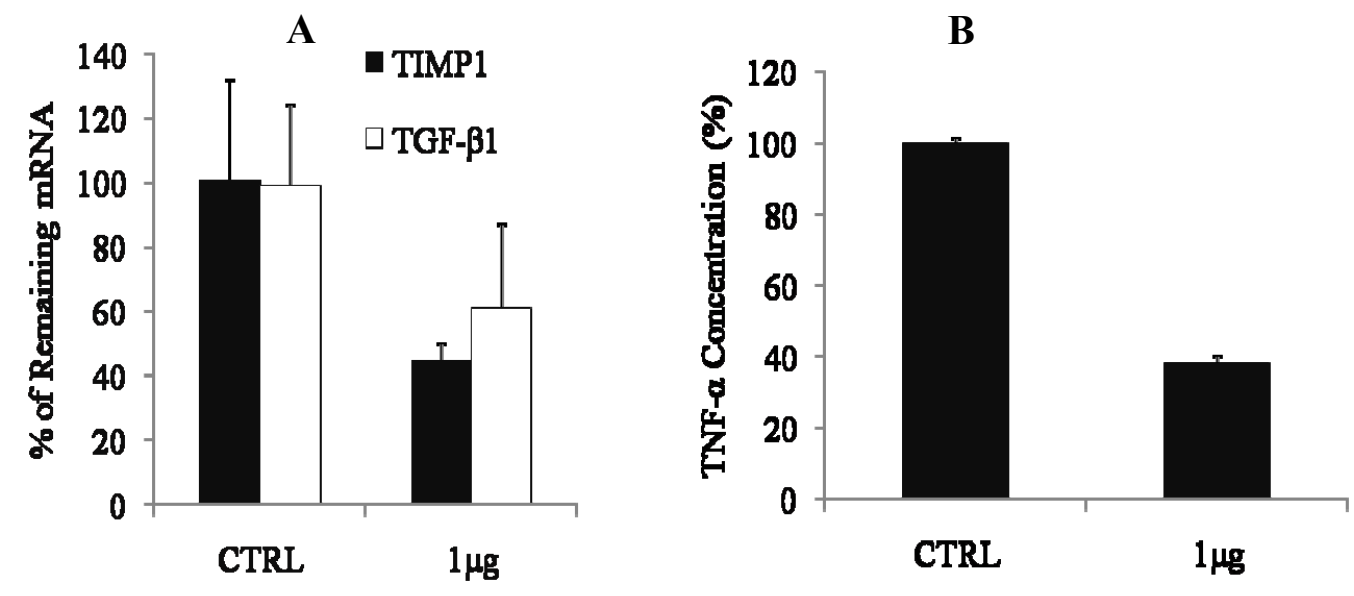

Figure 4-2 In vitro effect of TFO on HSC cells.

A) Effect on TIMP1 and TGF- $\beta 1$ expression after transfection of HSC-T6 cells with TFO after complex formation with lipofectamine 2000 . At $72 \mathrm{~h}$ post-transfection, cells were harvested, total RNA was extracted, and TIMP1 and TGF- $\beta 1$ expression was determined at mRNA levels using real time PCR. B) TNF- $\beta$ concentration in cultured medium was measured by enzyme-linked immunosorbent assay (ELISA). Data are expressed as the mean \pm standard deviation (SD). 


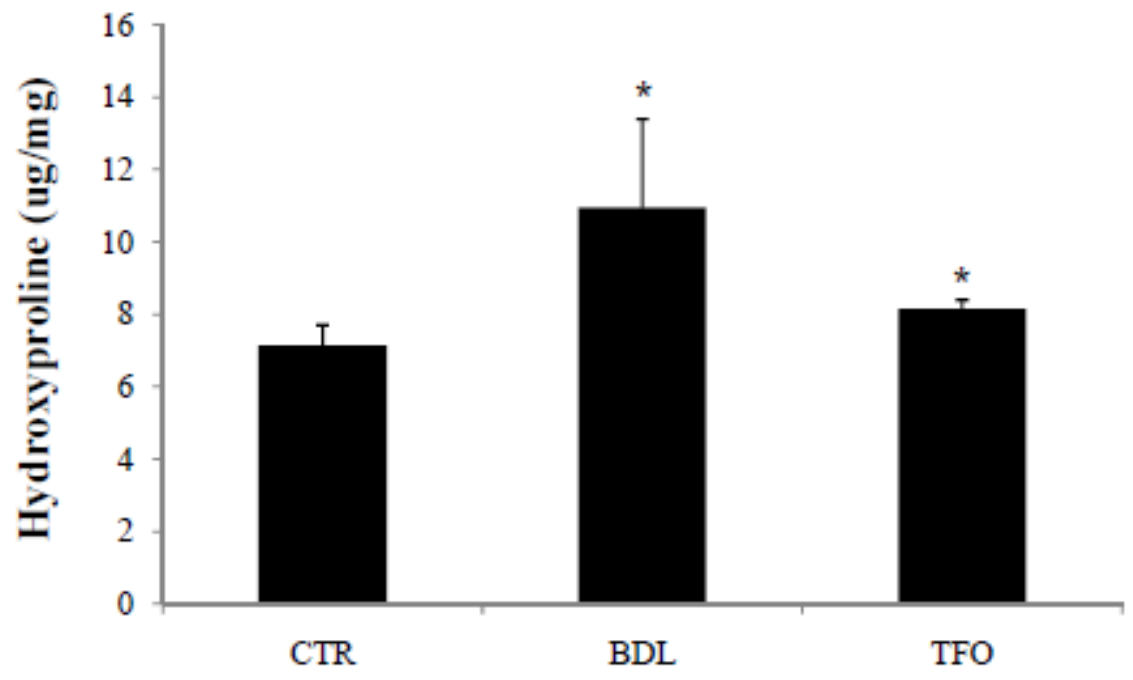

Figure 4-3 Hydroxyproline assay.

Total tissue hydroxyproline content was determined by biochemical assay at 7 weeks after BDL. Data are expressed as micrograms of hydroxyproline per milligram of tissue. Results are expressed as the mean \pm S.D. ${ }^{*} \mathrm{P}<0.05$ compared with control using ANOVA and t-test. TFO treatment reduces hepatic hydroxyproline content. 

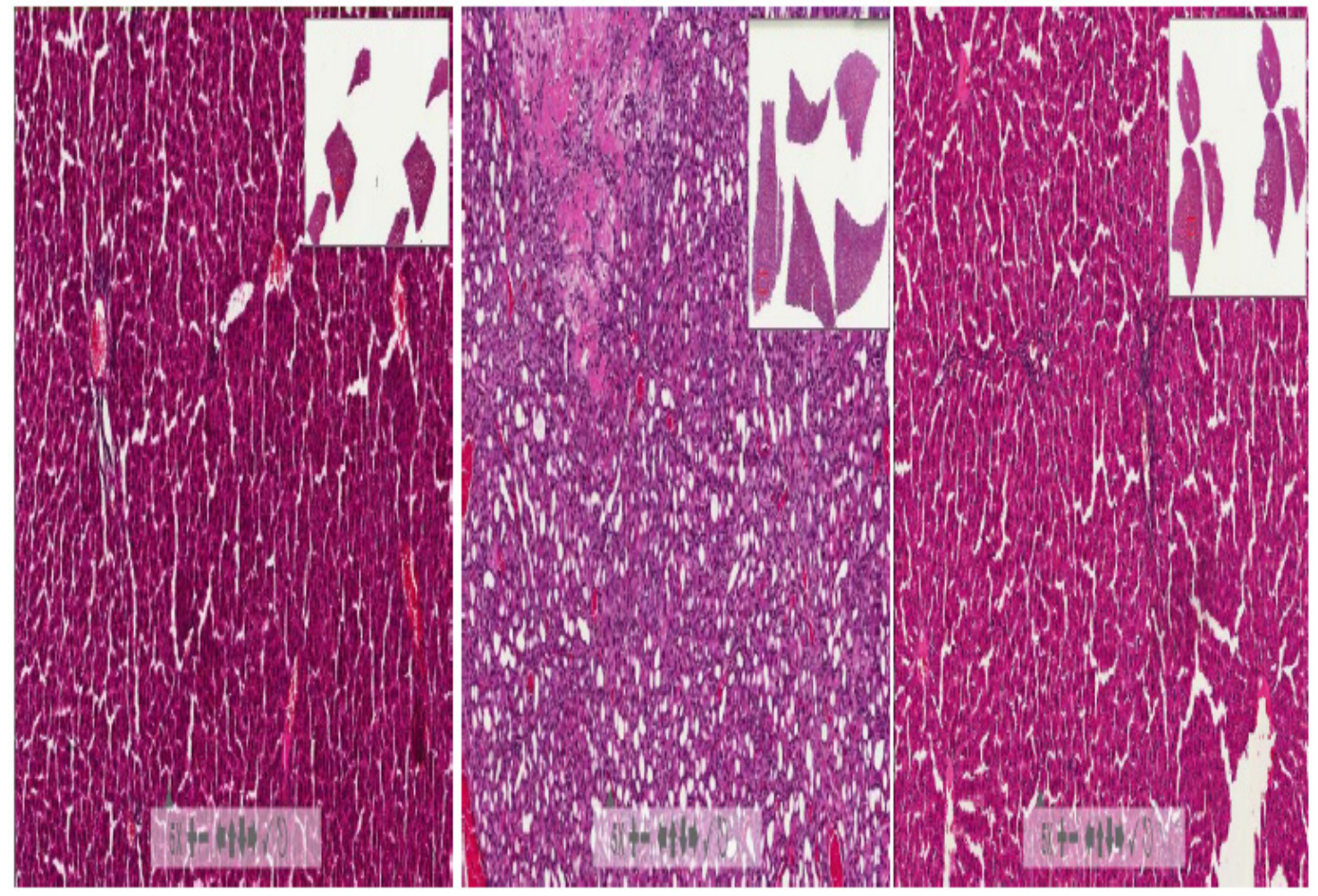

Figure 4-4 Hematoxylin and Eosin staining of livers sections.

TFO gene therapy attenuates liver fibrosis induced by BDL. Hematoxylin and Eosin staining staining of liver tissue sections from control, BDL and TFO-treated rats at 7 weeks after BDL. 

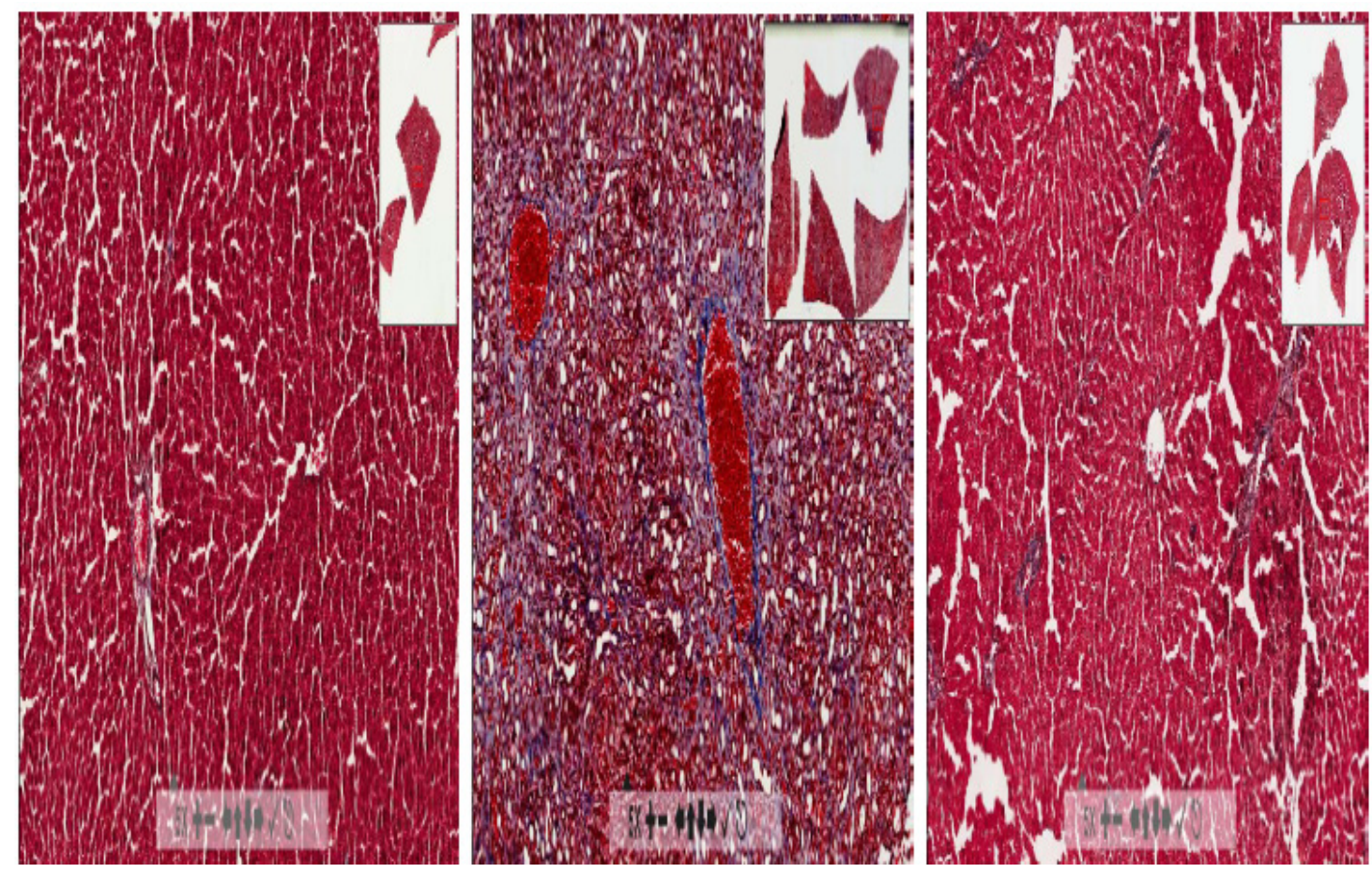

Figure 4-5 Masson's Trichrome staining for collagen.

TFO gene therapy attenuates liver fibrosis induced by BDL. Masson's trichrome staining of liver tissue sections from control, BDL and TFO-treated rats at 7 weeks after BDL. Significantly less hepatic collagen staining was observed in TFO-treated rats. 
confluent areas of necrotic hepatocytes in the collagenized zones. TFO administration, however, resulted in a marked reduction in the fibrotic stage indicated by decreasing portal and periportal accumulation of collagen and essentially no collagen accumulation was found in liver interstitium (Figure 4-5). Also, there was further reduction in branching of collagen in the liver sections of TFO treated rats compared to that of common bile duct ligated rats.

A computer-aided morphometric analysis revealed that the intensity of collagen staining in the liver sections at 7 weeks after CBDL was reduced by $60 \%$ after TFO treatment, when compared with the saline treated CBDL rats.

\subsubsection{Effect of TFO on Liver Functions and Serum Fibrotic Markers}

ALT is a cytosolic enzyme, primarily present in the liver. An increase in plasma ALT indicates liver damage more specifically than AST, which is a mitochondrial enzyme present in large quantities in the heart, liver, skeletal muscle, and kidney and in part indicates liver injury. At 7 weeks post CBDL, serum AST and ALT levels as determined by colorimetric assay were significantly higher in BDL animals with no TFO treatment compared with the CBDL rats treated with TFO (Figure 4-6).

\subsubsection{TFO Attenuates In Vivo Myofibroblast Activation}

To provide further evidence of the anti-fibrotic properties of TFO in CBDL rats, we used real time RT-PCR to determine the effects of this compound on hepatic $\alpha$-SMA protein expression, a well-established marker of HSC activation during liver fibrogenesis. ${ }^{200}$ Compared with sham controls, $\alpha$-SMA mRNA expression in the CBDL liver at 7 weeks was markedly increased, suggesting activation of the hepatic myofibroblasts following CBDL-induced injury. However, the induction of $\alpha$-SMA mRNA in the fibrotic liver was largely blocked by intravenous administration of TFO twice a week for four consecutive weeks at 3 weeks after CBDL (Figure 4-7). We next examined hepatic myofibroblast activation after CBDL by using immunofluorescence staining. In the normal rat liver, $\alpha$-SMA was exclusively and strongly expressed in vessel walls (portal vessels and centrilobular veins). The staining for $\alpha$-SMA was dramatically increased in CBDL liver forming whorls of layers (onion skinning) around the proliferated bile ductules. In addition to the peribiliary region in which $\alpha$-SMA positive cells were observed, strong $\alpha$-SMA staining was also found in enlarged and/or proliferated biliary epithelia (Figure 4-8). However, $\alpha$-SMA staining was fairly week in the fibrotic liver after intravenous administration of TFO twice a week for four consecutive weeks at 3 weeks after CBDL (Figure 4-8).

To confirm the epithelial origin of $\alpha$-SMA-positive cells, we used double immunofluorescence staining for biliary epithelial marker cytokeratin-19 (red) and $\alpha$ SMA (green). Figures 4-9 and 4-10 demonstrate clear colocalization of cytokeratin-19 and myofibroblast marker $\alpha-S M A$ in biliary epithelial cells at 7 weeks after CBDL. 

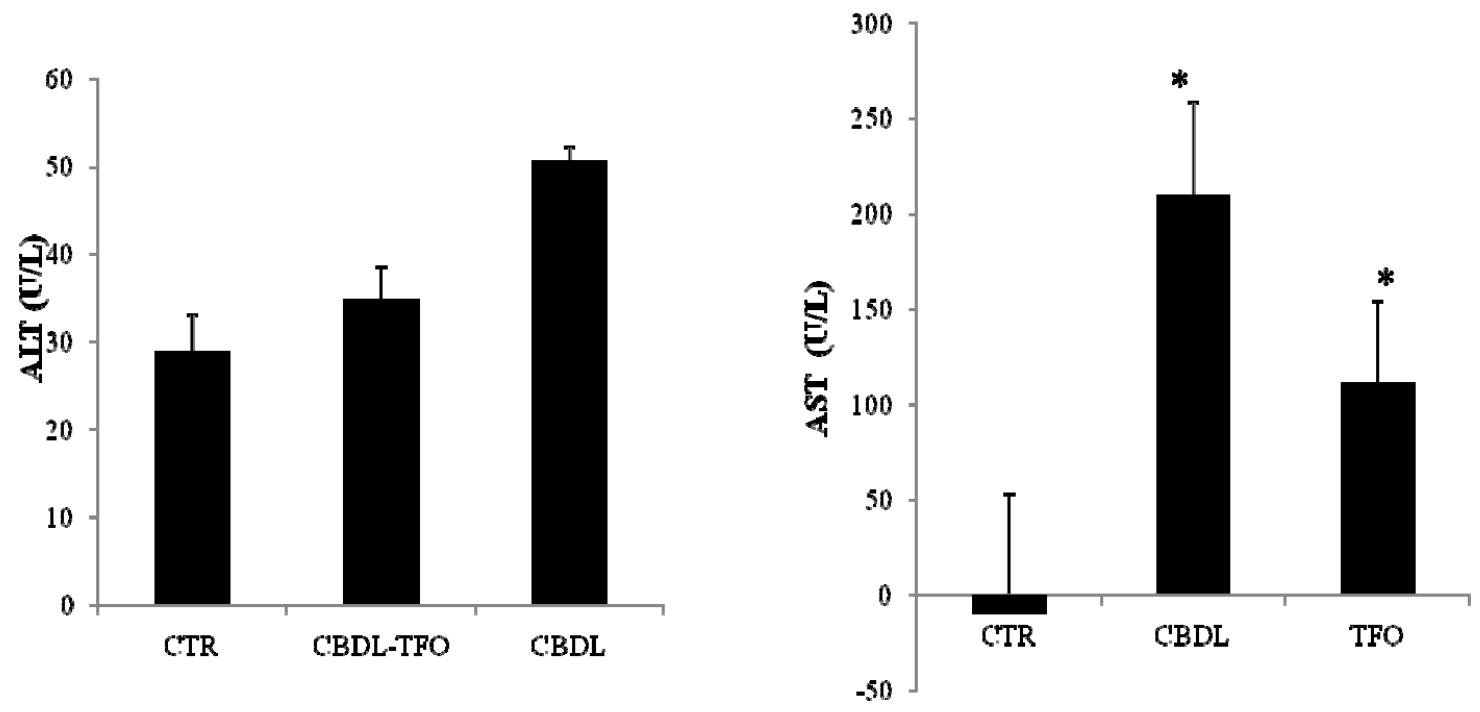

Figure 4-6 Serum ALT and AST assay.

Effect of TFO treatment on alanine aminotransferase (ALT) and aspartate aminotransferase (AST) content. ALT and AST levels were determined by colorimetric assay at 7 weeks after CBDL in rats TFO treatment reduces hepatic ALT and AST content compared to CBDL rats without any TFO treatment. Results are expressed as the mean \pm S.D. ${ }^{*} \mathrm{P}<0.05$ compared with control using ANOVA. 


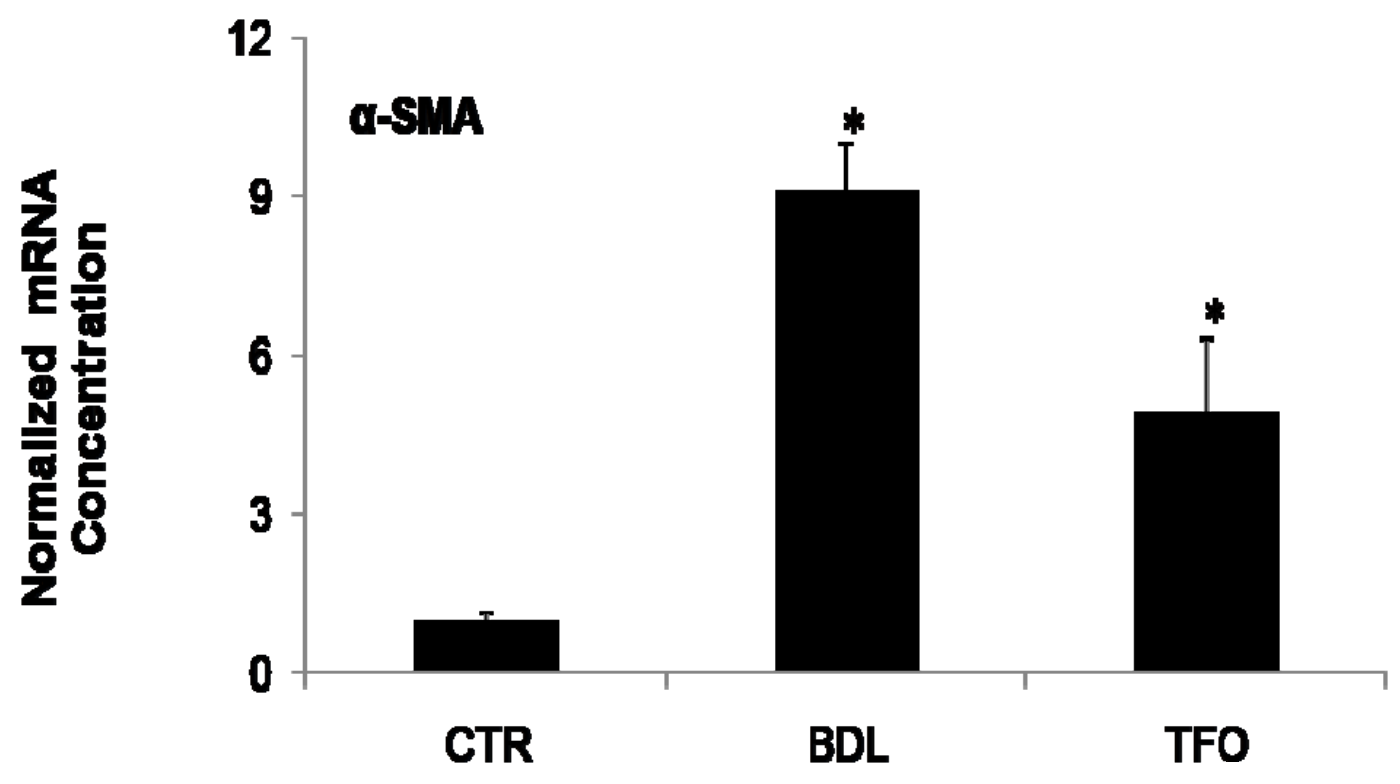

Figure 4-7 Real time PCR analysis for $\alpha$-SMA.

Reverse transcription-PCR showed an inhibitory effect of TFO on $\alpha$-collagen expression levels in BDL rats. The mRNA levels of $\alpha$-collagen and $\alpha$-SMA was determined by reverse transcription-PCR 7 weeks after BDL. ${ }^{*} \mathrm{P}<0.05$ compared with control using ANOVA. 

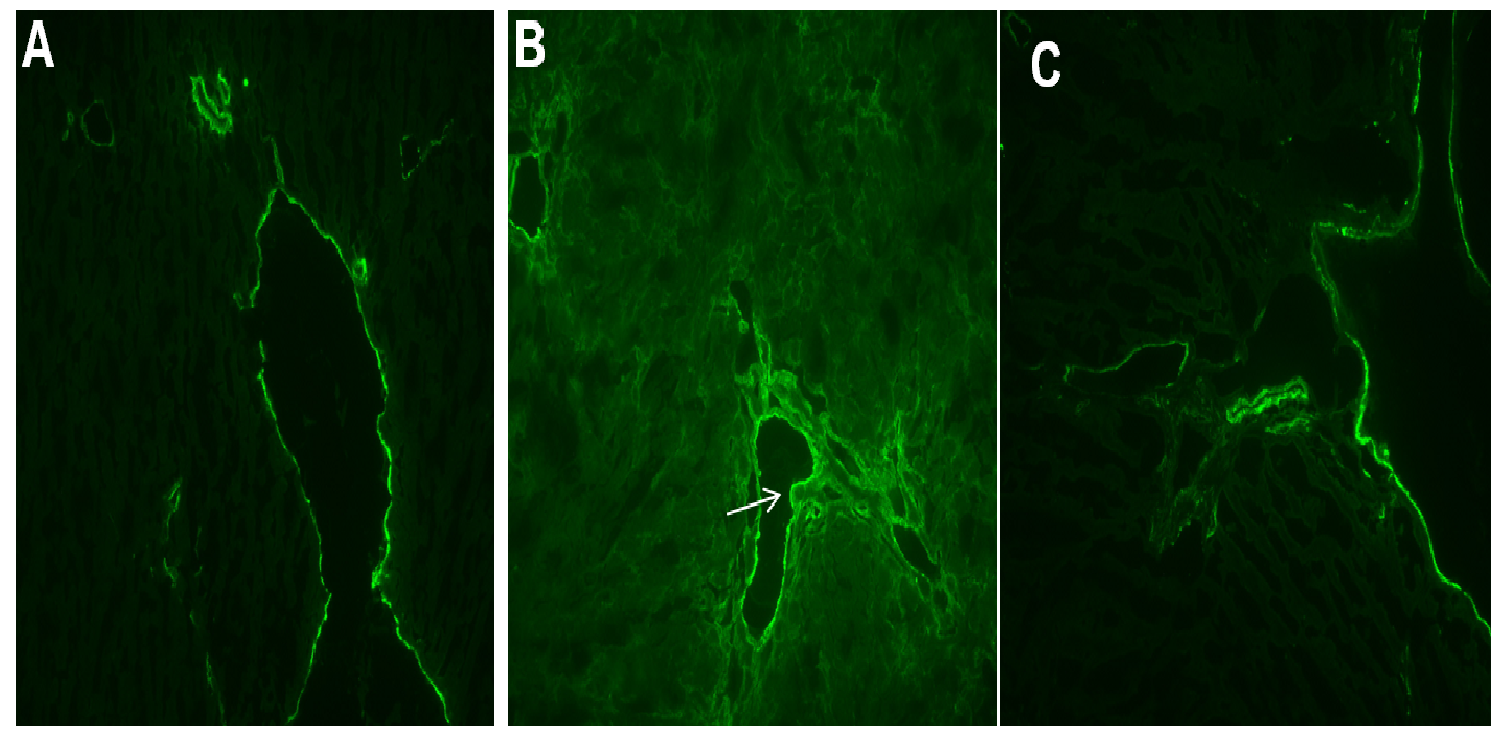

Figure 4-8 Immunoflorescent staining for $\alpha$-SMA.

Immunoflorescent staining for $\alpha$-SMA in A) Control B) BDL and C) TFO treated liver sections at $10 \mathrm{X}$ magnification respectively. Arrows indicate the positive staining for $\alpha$-SMA in and proliferated bile ductules. 


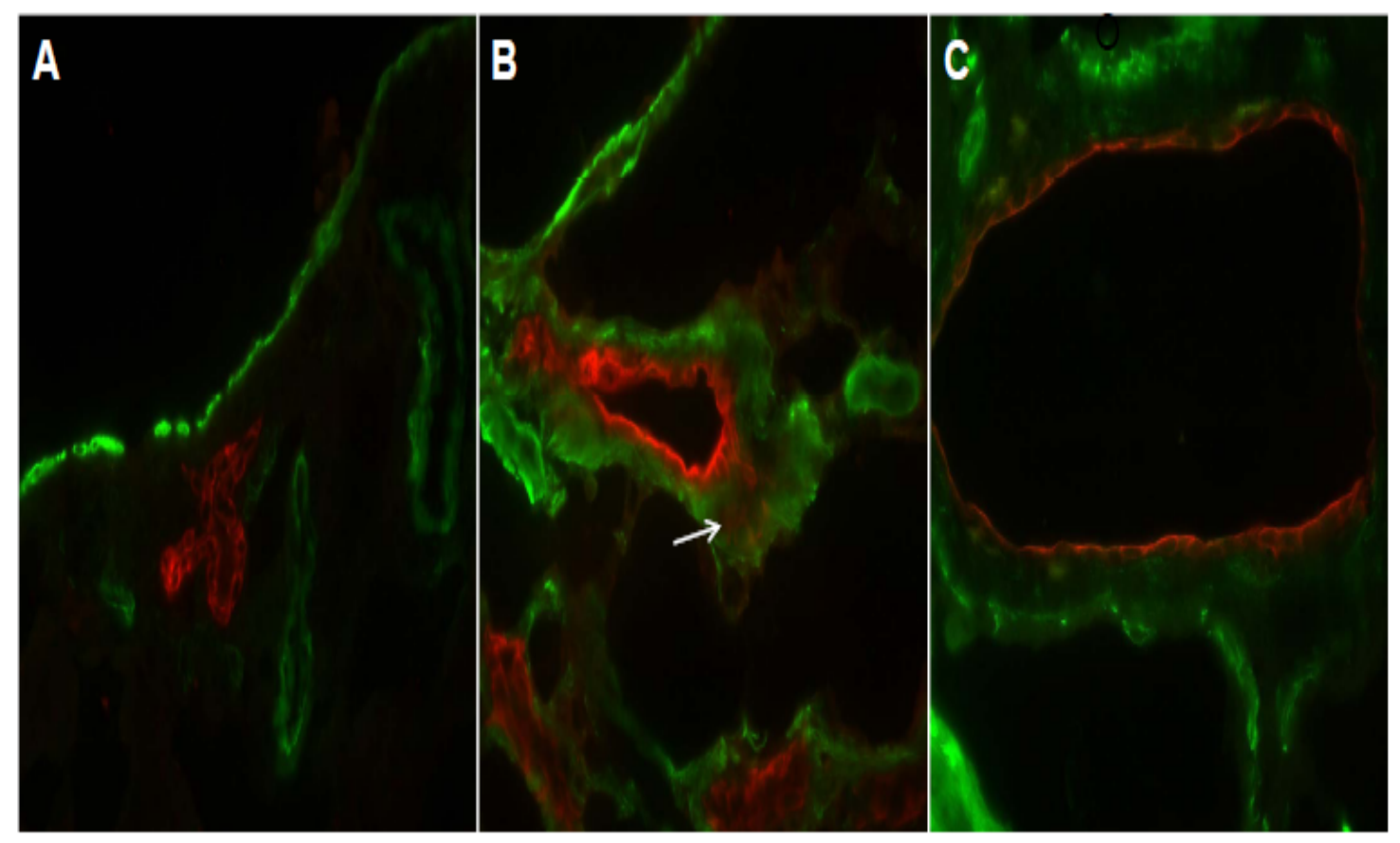

Figure 4-9 Double immunostaining for cytokeratin-19 and $\alpha$-SMA.

Double immunostaining for cytokeratin-19 and $\alpha$-SMA in A) Control B) BDL and C) TFO treated liver sections. The arrows indicate the colocalization of cytokeratin-19 and $\alpha$-SMA indicating the phenomenon of epithelial-mesenchymal cell transition (EMT) occurring in and around the peribiliary space. 


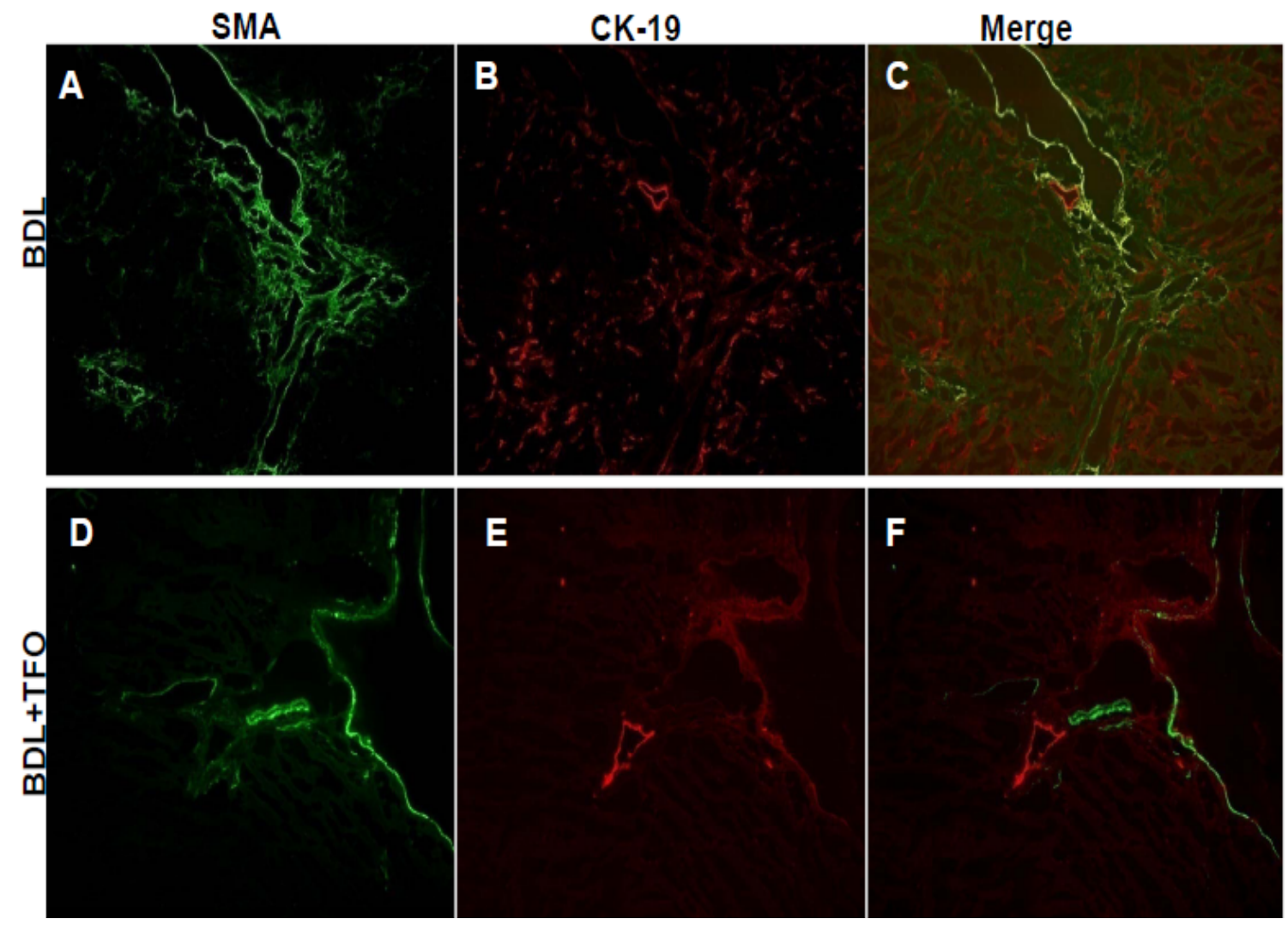

Figure 4-10 Colocalization of Cytokeratin-19 and $\alpha$-SMA.

Double immunostaining for cytokeratin-19 and $\alpha$-SMA in A\&B) BDL and C\&D) TFO treated liver sections. Merging of cytokeratin-19 and $\alpha-S M A$ is shown in C\&F. All the images are at $10 \mathrm{X}$ magnification. 
Exogenous TFO, however, prevented the coexpression of cytokeratin-19 and $\alpha$-SMA in biliary epithelium. These observations imply that biliary epithelial cells may undergo a phenotypic transition into myofibroblasts under pathological conditions and that exogenous TFO administration could protect the biliary epithelia by blocking this phenotypic transition.

\subsubsection{TFO Inhibits Neutrophil Infiltration by Downregulating Inflammatory Cytokines}

CBDL may cause hepatocyte apoptosis due to TNF- $\alpha$ upregulation and consequent neutrophil infiltration. Therefore, we determined the amount of TNF- $\alpha$ secreted from the livers of TFO treated and non-treated CBDL rats. There was only slight elevation in TNF- $\alpha$ level in the non-treated BDL group indicating only minor inflammation following the induction of liver fibrosis by bile duct ligation (Figure 4-11A). The level of myeloperoxidase (MPO) which is a marker for neutrophil infiltration was less in the TFO treated CBDL group compared to non-treated CBDL group, indicating that TFO treatment can decrease neutrophilic infiltration by inhibiting the release of inflammatory cytokine such as TNF- $\alpha$ (Figure 4-11B).

\subsection{Discussion}

Fibrosis is characterized by an excessive production of ECM components, especially Type I collagen, and if not controlled it can lead to organ dysfunction. ${ }^{201}$ Therefore, inhibition of collagen synthesis should prevent fibrosis. Joseph et al., demonstrated that antiparallel polypurine phosphorothioate oligonucleotides could form triplexes with the $\mathrm{C} 1$ region of the $\alpha 1$ (I) collagen gene promoter and inhibit transcription of $\alpha 1$ (I) collagen gene promoter activity in rat fibroblasts in culture. ${ }^{82} \mathrm{We}$ determined triplex formation of a psoralen modified TFO with $\alpha$ 1(I) collagen gene in isolated nuclei and intact HSC-T6 cells, and demonstrated strong correlation between triplex formation and transcription inhibition of TFOs. ${ }^{191}$ HSCs are the key fibrogenic cells and upon activation they convert into myofibroblast- like cells, excrete excess ECM components, and overexpress TGF- $\beta 1$ and $\alpha$-SMA, which are markers for myofibroblasts and fibronectin, respectively. ${ }^{202}$ Therefore, in our previous studies, we isolated different liver cells after systemic administration of TFO and demonstrated almost $40 \%$ of the total liver recovery was in the HSCs. ${ }^{203}$ In the present study, we investigated the therapeutic effect of TFO on liver fibrosis in rats induced by CBDL, which is a commonly used animal model.

The serum TNF- $\alpha$ levels were elevated following CBDL, and was noted to be lower in the TFO treated group (Figure 4-11A). TNF- $\alpha$ is an important mediator of hepatotoxicity and is upregulated in several animal models of chronic and acute liver injury. Gabele et al., have studied the effect of CBDL on TNF- $\alpha$ knockout mice and found them to be more resilient to the liver injury secondary to CBDL. ${ }^{204}$ They also showed that TNF- $\alpha$ knockout mice had a significant decrease in expression of collagen, $\alpha$-SMA and TGF- $\beta 1$ upon induction of fibrosis by BDL. Histopathological examination 


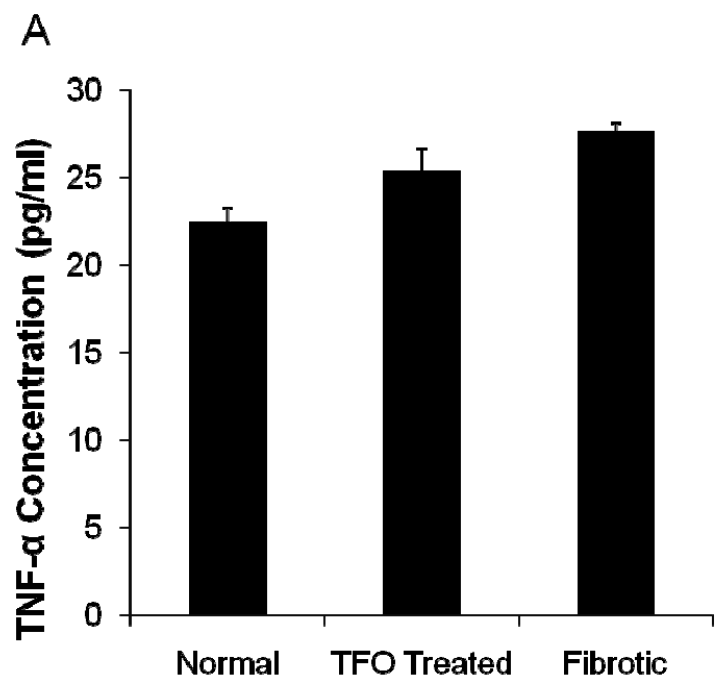

B

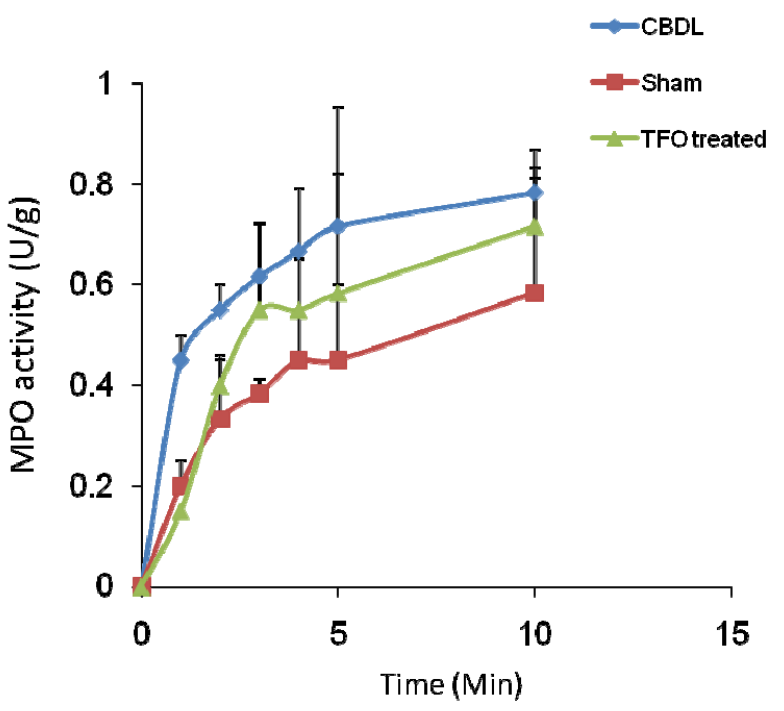

Figure 4-11 TNF- $\alpha$ ELISA and Myeloperoxidase assay.

A) Effect of in vivo TFO treatment on TNF- $\alpha$. Rat serum was separated from the plasma. Following separation, TNF- $\alpha$ in serum were measured by enzyme-linked immunosorbent assay (ELISA). B) Myeloperoxidase (MPO) activity in the liver tissue of CBDL sham and TFO treated Time (Min) ELISA). CBDL, groups. One unit of MPO activity was defined as the amount of the MPO in 1 gram of liver tissue that could change $1 \mathrm{OD}$ in absorbance at $460 \mathrm{~nm}$. 
of the liver tissue using Masson's trichrome staining also revealed a decrease in staining after treatment with TFO twice a week for 4 consecutive weeks at 3 weeks post CBDL (Figure 4-5). In addition, the TFO treated group also showed less neutrophilic infiltration compared to the BDL group suggesting the attenuation of liver fibrosis. The increase in concentration of ALT and AST levels seen following BDL also showed a decreasing trend after TFO treatment indicating reduction of hepatic inflammation. Xia et al., have shown that mesenchymal cell transition (EMT) plays a vital role in the development of liver fibrosis due to CBDL. ${ }^{205}$ TGF- $\beta 1$ has also been shown to induce EMT transition to induce idiopathic pulmonary fibrosis. ${ }^{186}$ There have been reports, which suggest that the cultured neonatal rat hepatocytes undergo EMT at specific conditions. ${ }^{206}$ It will not be surprising if BECs undergo phenotypic transition to matrix-producing myofibroblasts after CBDL. It has also been seen that there is a decrease in the hepatic myofibroblast activation and biliary fibrosis when EMT was blocked by hepatocyte growth factor. ${ }^{205}$ TGF- $\beta 1$ is also an important factor resulting in EMT resulting in fibrosis. There are several reports in the literature which have showed that TGF- $\beta 1$ can induce EMT upregulation of Smad-interacting protein-1 (SIP1) and Snail..$^{206-208}$ In the present study, TGF- $\beta 1$ levels were increased significantly following BDL as seen by Western blot. Treatment with TFO showed a significant reduction in TGF- $\beta 1$ levels. (data not shown) These findings suggest that the transformed HSCs possibly secreted TGF- $\beta 1$. This is also consistent with the current idea that HSCs are transformed into myofibroblasts and secrete TGF- $\beta 1$, thus stimulating the production of ECM, and probably their proliferation, through a paracrine/autocrine loop.

In the present study, we have shown that CBDL results in the transformation of biliary epithelial cells (BECs) to $\alpha$-SMA expressing myofibroblasts which migrate into the periductal region through the impaired basement membrane. Most of BECs around the bile duct in CBDL rats were found positive for $\alpha$-SMA (Figure 4-8) corroborating the fact that EMT may be the phenomenon behind the development of myofibroblasts. There was also considerable co-localization of both $\alpha$-SMA and Cytokeratin-19 in the interstitium around the bile duct suggesting migration of newly formed myofibroblasts (Figures 4-9 and 4-10).

TFO treatment can inhibit the transcription of Type I collagen and also decrease in $\alpha$ SMA levels in TFO treated group, suggesting that the inhibition of collagen can also lead to down regulation of $\alpha$-SMA levels. This helps in attenuating fibrosis induced by CBDL. Furthermore, it also inhibits the EMT, thereby decreasing the development of profibrogenic cells and inhibiting the accumulation of ECM. This study shows that a TFO can have a beneficial effect on decreasing liver fibrosis and reversing progression of liver injury. Further studies would be required to understand the exact mechanism by which the TFO inhibits EMT. 


\section{CHAPTER 5. SUMMARY}

Transplantation therapy for diabetes is limited by the toxicity of immunosuppressive drugs. However, even if toxicity can be minimized, there will still be a shortage of human donor organs. Ex vivo transplantation of islets can be a viable alternative for treating diabetes. Transplantation of islets is a viable therapy for treating Diabetes. However, there are certain problems that need to be overcome. The major problems for islet transplantation are lack of revascularization following transplantation and the nonfunction of islet grafts due to immune responses following transplantation. We used adenoviral vectors encoding VEGF/HGF and IL-1Ra genes which can help in revascularization of islets and protect the islets from immune mediated destruction.

We constructed Adenoviral vector encoding VEGF and IL-1Ra. Initially we confirmed the expression of the genes, VEGF and IL-1Ra following transduction in human islets. There was very high expression of these genes from the Adv transduced islets compared to non-transduced islets. We further checked the functionality and viability of islets following Adv transduction. The stimulation indices of Adv transduced islets were similar to the non-transduced islets indication no detrimental effects from the Adv. Following this, we studied the effect of IL-1Ra in protecting the islets from apoptosis by incubating the Adv treated islets in a cytokine cocktail. The caspase- 3 levels were less compared to that of the islets with no Adv transduction. We then transplanted islets transduced with the Adv-hVEGF-hIL-1Ra in the kidney capsules of NOD-SCID diabetic mice. The islets transduced with Adv showed better metabolic control and functionality and were able to lower the blood glucose levels for a long period of time compared to mice transplanted with non-transduced islets or control vector Adv- Lacz. There was also an increase in expression of insulin and c-peptide in mice transplanted with Adv compared to mice transplanted with non-transduced islets.

Immunohistochemical staining of kidney sections revealed extensive staining for insulin and human vonWillebrand factor (marker for endothelial cells) in Adv-hVEGF-hIL-1Ra transduced islets. This shows that the bicistronic adenoviral vector indeed expressed the genes VEGF and IL-1Ra and was able to decrease the blood glucose levels in the Diabetic NOD-SCID mice.

We then decided to replace VEGF with HGF as HGF can not only promote revascularization of islets, but also can further promote $\beta$ - cell proliferation, function and survival following transplantation. HGF gene therapy also showed reduction in minimum islet transplant mass required for transplantation. Adenoviral-mediated gene transfer of HGF has been shown to improve islet transplant outcomes. HGF is also known to inhibit apoptosis. We constructed Adv-hHGF-hIL-1Ra and transduced them to human islets to see the expression of HGF and IL-1Ra. There was dose and time dependent expression of both the genes from the vector. The Adv vectors were relatively safe at lower dose of around $1000 \mathrm{MOI}$ with the stimulation index being similar to that of control nontransduced islets. Then we studied the effect of Adv in protecting the islets from apoptosis. The islets transduced with Adv-hHGF-hIL-1Ra showed less expression of caspase-3 compared to control indicating the effectiveness of $\mathrm{Adv}$ in protection from 
apoptosis. Transplantation of islets transduced with Adv-hHGF-hIL-1Ra showed better metabolic function and decreased the blood glucose levels for a longer time compared to NOD-SCID mice transplanted with non-transduced islets. Immunohistochemical staining of kidney sections showed extensive staining for insulin and $\mathrm{hVw}$ factor for $\mathrm{Adv}$ transplanted islets compared to that of non-transplanted control. At the end of in vivo study, intraperitoneal glucose tolerance test (IPGTT) was done to study the metabolic function of transplanted islets. The mice transplanted with Adv transduced islets behave well compared to those with non-transduced islets.

In summary bipartite Adenoviral vectors which are replication-deficient can be used successfully for transducing islets for improving the islet transplantation outcomes. We saw a decrease in blood glucose levels in NOD-SCID mice transplanted with Adv transduced islets and further these islets were able to decrease the blood glucose levels for a sustained period of time. Adv vectors were relatively safe at MOI up to 1000 and showed efficient expression of VEGF, HGF and IL-1Ra corroborating the literature. More studies reflecting the immune responses from these vectors need to be studied to further confirm the beneficial effects of these vectors in islet transplantation. Further, HGF thereapy can decrease the number of islets needed for transplantation, which is very crirtical as there is an enormous shortage in islets required to attain euglycemia.

Liver fibrosis is a result of accumulation of fibrous, scar tissue in the liver. It usually occurs due to the injury to liver cells (hepatocytes) by trauma, viral infection, excessive alcohol consumption, toxins or other factors that lead to increase in activation of immune system and resulting in the excessive accumulation of ECM. The hepatic stellate cells (HSCs) get activated resulting in inflammation of hepatocytes causing excessive accumulation of Type I collagen and ECM. It is often associated with high morbidity and mortality and can lead to the need for a liver transplant. There has been no proper therapy for liver fibrosis till now. Bile duct ligation is the most frequently used method in developing fibrosis. We have evaluated the use of TFOs inhibiting Type I collagen in attenuating fibrosis induced by bile duct ligation. BDL is a commonly used animal model for inducing fibrosis in liver. Biliary fibrosis can be caused either due to biliary atresia or primary sclerosing cholangitis. The injury to the bile duct by performing BDL may trigger a chain of events resulting in the development of fibrosis.

We have shown that BDL results in the transformation of BECs to $\alpha$-SMA expressing myofibroblasts which apparently migrate into the periductal region through the impaired basement membrane. Most of the BECs around the bile duct in BDL rats were found positive for $\alpha$-SMA corroborating the fact that EMT may be the phenomenon behind the development of myofibroblasts. Further, colocalization of $\alpha$-SMA and Cytokeratin-19 in the BECs confirmed the phenotypic transition of BECs to mesenchymal cells. There was considerable colocalization of both $\alpha$-SMA and Cytokeratin-19 in the interstitium around the bile duct signifying the migration of newly formed myofibroblasts. This phenomenon was mitigated in the animals treated with TFO inhibiting Type I collagen. TGF- $\beta 1$ can be an important factor resulting in biliary EMT of hepatocytes resulting in fibrosis. TGF$\beta 1$ levels were increased significantly following BDL as seen by western blot and Real time PCR analysis. Treatment with TFO showed a significant reduction in the TGF- $\beta 1$ 
levels. There was also a decrease in the $\alpha$-SMA levels in the TFO treated group compared to the non-treated BDL group suggesting that the inhibition of collagen can also lead to down regulation of $\alpha$-SMA levels.

The hydroxyproline levels in TFO treated group was very less compared to the BDL group. This is in line with our previous studies suggesting the inhibition of transcription of Type I collagen. The serum TNF- $\alpha$ levels were elevated following BDL, and was higher than that in the TFO treated group. TNF- $\alpha$ is a mediator of hepatotoxicity and is upregulated in several animal models of chronic and acute liver injury. Masson's trichrome staining revealed a significantly high staining for collagen in liver sections obtained from BDL group which decreased after treatment with TFO. There was also seen an increase in concentrations of ALT and AST levels following BDL. TFO treatment showed a decrease in the concentrations of the ALT and AST levels indicating the effectiveness of TFO in attenuating liver fibrosis.

TFO treatment inhibiting the transcription of Type I collagen helps in attenuating fibrosis induced by BDL. It also further inhibits the EMT, thereby decreasing the development of profibrogenic cells and inhibiting the accumulation of ECM. Although, the mechanism of TFO inhibiting the EMT needs to be thoroughly investigated, this study shows the beneficial effects of TFO and it can be a good candidate for treating fibrotic disorders. 


\section{LIST OF REFERENCES}

1. Butler AE et al. Beta-cell deficit and increased beta-cell apoptosis in humans with type 2 diabetes. Diabetes 2003; 52: 102-110.

2. Rahier J et al. Pancreatic beta-cell mass in European subjects with type 2 diabetes. Diabetes Obes Metab 2008; 10 Suppl 4: 32-42.

3. Ojo AO et al. The impact of simultaneous pancreas-kidney transplantation on long-term patient survival. Transplantation 2001; 71: 82-90.

4. Larsen JL. Pancreas transplantation: indications and consequences. Endocr Rev 2004; 25: 919-946.

5. Lanza RP, Sullivan SJ, Chick WL. Perspectives in diabetes. Islet transplantation with immunoisolation. Diabetes 1992; 41: 1503-1510.

6. Soon-Shiong P et al. Successful reversal of spontaneous diabetes in dogs by intraperitoneal microencapsulated islets. Transplantation 1992; 54: 769-774.

7. Narang AS, Mahato RI. Biological and biomaterial approaches for improved islet transplantation. Pharmacol Rev 2006; 58: 194-243.

8. Makhlouf $\mathrm{L}$ et al. The role of autoimmunity in islet allograft destruction: major histocompatibility complex class II matching is necessary for autoimmune destruction of allogeneic islet transplants after T-cell costimulatory blockade. Diabetes 2002; 51: 3202-3210.

9. Mahato RI, Rolland A, Tomlinson E. Cationic lipid-based gene delivery systems: pharmaceutical perspectives. Pharm Res 1997; 14: 853-859.

10. van Gaal EV, Hennink WE, Crommelin DJ, Mastrobattista E. Plasmid engineering for controlled and sustained gene expression for nonviral gene therapy. Pharm Res 2006; 23: 1053-1074.

11. Calcedo $\mathrm{R}$ et al. Host immune responses to chronic adenovirus infections in human and nonhuman primates. J Virol 2009; 83: 2623-2631

12. Sangro B, Herraiz M, Prieto J. Gene therapy of neoplastic liver diseases. Int $J$ Biochem Cell Biol 2003; 35: 135-148.

13. Cheng K, Yang N, Mahato RI. TGF-betal gene silencing for treating liver fibrosis. Mol Pharm 2009; 6: 772-779.

14. Brown T. Gene Cloning: an Introduction. Chapman \& Hall: London, 1990.

15. Jang SK et al. A segment of the 5' nontranslated region of encephalomyocarditis virus RNA directs internal entry of ribosomes during in vitro translation. $J$ Virol 1988; 62: 2636-2643.

16. Pelletier J, Sonenberg N. Internal initiation of translation of eukaryotic mRNA directed by a sequence derived from poliovirus RNA. Nature 1988; 334: 320-325.

17. Baranick BT et al. Splicing mediates the activity of four putative cellular internal ribosome entry sites. Proc Natl Acad Sci U S A 2008; 105: 4733-4738.

18. Jia X, Cheng K, Mahato RI. Coexpression of vascular endothelial growth factor and interleukin-1 receptor antagonist for improved human islet survival and function. Mol Pharm 2007; 4: 199-207.

19. Voorhees JJ, Duell EA, Chambers DA, Marcelo CL. Regulation of cell cycles. $J$ Invest Dermatol 1976; 67: 15-19. 
20. Lund $\mathrm{J}$ et al. Transcriptional regulation of the bovine CYP17 gene by cAMP. Steroids 1997; 62: 43-45.

21. Dillon N. Gene regulation and large-scale chromatin organization in the nucleus. Chromosome Res 2006; 14: 117-126.

22. Hoffmann D, Wildner O. Efficient generation of double heterologous promoter controlled oncolytic adenovirus vectors by a single homologous recombination step in Escherichia coli. BMC Biotechnol 2006; 6: 36.

23. Gambari R. New trends in the development of transcription factor decoy (TFD) pharmacotherapy. Curr Drug Targets 2004; 5: 419-430.

24. Melloul D, Marshak S, Cerasi E. Regulation of pdx-1 gene expression. Diabetes 2002; 51 Suppl 3: S320-325.

25. Kawasaki $\mathrm{H}$ et al. In vitro transformation of adult rat hepatic progenitor cells into pancreatic endocrine hormone-producing cells. J Hepatobiliary Pancreat Surg 2008; 15: 310-317.

26. Naujok $\mathrm{O}$ et al. Changes in gene expression and morphology of mouse embryonic stem cells on differentiation into insulin-producing cells in vitro and in vivo.

Diabetes Metab Res Rev 2009; 25: 464-476.

27. Walther W, Stein U. Cell type specific and inducible promoters for vectors in gene therapy as an approach for cell targeting. J Mol Med 1996; 74: 379-392.

28. Spilianakis CG et al. Interchromosomal associations between alternatively expressed loci. Nature 2005; 435: 637-645.

29. Arnosti DN, Kulkarni MM. Transcriptional enhancers: intelligent enhanceosomes or flexible billboards? J Cell Biochem 2005; 94: 890-898.

30. Choi UH et al. Hypoxia-inducible expression of vascular endothelial growth factor for the treatment of spinal cord injury in a rat model. J Neurosurg Spine 2007; 7: 54-60.

31. Mahato RI, Smith LC, Rolland A. Pharmaceutical perspectives of nonviral gene therapy. Adv Genet 1999; 41: 95-156.

32. Smale ST, Kadonaga JT. The RNA polymerase II core promoter. Annu Rev Biochem 2003; 72: 449-479.

33. Qin L et al. Promoter attenuation in gene therapy: interferon-gamma and tumor necrosis factor-alpha inhibit transgene expression. Hum Gene Ther 1997; 8: 20192029.

34. Lubansu A et al. Recombinant AAV viral vectors serotype 1, 2, and 5 mediate differential gene transfer efficiency in rat striatal fetal grafts. Cell Transplant 2008; 16: 1013-1020.

35. Yew NS. Controlling the kinetics of transgene expression by plasmid design. $A d v$ Drug Deliv Rev 2005; 57: 769-780.

36. Tenenbaum L et al. Recombinant AAV-mediated gene delivery to the central nervous system. $J$ Gene Med 2004; 6 Suppl 1: S212-222.

37. Alexopoulou AN, Couchman JR, Whiteford JR. The CMV early enhancer/chicken beta actin $(\mathrm{CAG})$ promoter can be used to drive transgene expression during the differentiation of murine embryonic stem cells into vascular progenitors. BMC Cell Biol 2008; 9: 2.

38. Huang $\mathrm{J}$ et al. Myocardial injection of CA promoter-based plasmid mediates efficient transgene expression in rat heart. J Gene Med 2003; 5: 900-908. 
39. Gill DR et al. Increased persistence of lung gene expression using plasmids containing the ubiquitin C or elongation factor 1alpha promoter. Gene Ther 2001; 8: $1539-1546$.

40. Chen NK, Tan SY, Udolph G, Kon OL. Insulin expressed from endogenously active glucose-responsive EGR1 promoter in bone marrow mesenchymal stromal cells as diabetes therapy. Gene Ther 2010; 17: 592-605.

41. Kozak M. Structural features in eukaryotic mRNAs that modulate the initiation of translation. J Biol Chem 1991; 266: 19867-19870.

42. Kozak M. Regulation of translation in eukaryotic systems. Annu Rev Cell Biol 1992; 8: 197-225.

43. Hartikka J et al. An improved plasmid DNA expression vector for direct injection into skeletal muscle. Hum Gene Ther 1996; 7: 1205-1217.

44. Gregor PD, Kobrin BJ, Milcarek C, Morrison SL. Sequences 3' of immunoglobulin heavy chain genes influence their expression. Immunol Rev 1986; 89: 31-48.

45. Ryu WS, Mertz JE. Simian virus 40 late transcripts lacking excisable intervening sequences are defective in both stability in the nucleus and transport to the cytoplasm. J Virol 1989; 63: 4386-4394.

46. Esposito D, Chatterjee DK. Enhancement of soluble protein expression through the use of fusion tags. Curr Opin Biotechnol 2006; 17: 353-358.

47. Niiranen L et al. Comparative expression study to increase the solubility of cold adapted Vibrio proteins in Escherichia coli. Protein Expr Purif 2007; 52: 210218.

48. Shih YP et al. High-throughput screening of soluble recombinant proteins. Protein Sci 2002; 11: 1714-1719.

49. Mahato RI et al. Biodistribution and gene expression of lipid/plasmid complexes after systemic administration. Hum Gene Ther 1998; 9: 2083-2099.

50. Ma X, Riemann H, Gri G, Trinchieri G. Positive and negative regulation of interleukin-12 gene expression. Eur Cytokine Netw 1998; 9: 54-64.

51. Fink DJ, DeLuca NA, Goins WF, Glorioso JC. Gene transfer to neurons using herpes simplex virus-based vectors. Annu Rev Neurosci 1996; 19: 265-287.

52. Snowden BW, Blair ED, Wagner EK. Transcriptional activation with concurrent or nonconcurrent template replication has differential effects on transient expression from herpes simplex virus promoters. Virus Genes 1989; 2: 129-145.

53. Black J, Vos JM. Establishment of an oriP/EBNA1-based episomal vector transcribing human genomic beta-globin in cultured murine fibroblasts. Gene Ther 2002; 9: 1447-1454.

54. Kolb AF et al. Site-directed genome modification: nucleic acid and protein modules for targeted integration and gene correction. Trends Biotechnol 2005; 23: 399-406.

55. Van Craenenbroeck K, Vanhoenacker P, Haegeman G. Episomal vectors for gene expression in mammalian cells. Eur J Biochem 2000; 267: 5665-5678.

56. Ehrhardt A et al. Optimization of cis-acting elements for gene expression from nonviral vectors in vivo. Hum Gene Ther 2003; 14: 215-225.

57. Zhao $\mathrm{H}$ et al. Contribution of Toll-like receptor 9 signaling to the acute inflammatory response to nonviral vectors. Mol Ther 2004; 9: 241-248. 
58. Li A et al. A therapeutic DNA vaccination strategy for autoimmunity and transplantation. Vaccine 2010; 28: 1897-1904.

59. Chattopadhyay S, Pavithra L. MARs and MARBPs: key modulators of gene regulation and disease manifestation. Subcell Biochem 2007; 41: 213-230.

60. Boulikas T. Homeotic protein binding sites, origins of replication, and nuclear matrix anchorage sites share the ATTA and ATTTA motifs. J Cell Biochem 1992; 50: 111-123.

61. Guy-Caffey JK et al. Novel polyaminolipids enhance the cellular uptake of oligonucleotides. J Biol Chem 1995; 270: 31391-31396.

62. Argyros $\mathrm{O}$ et al. Persistent episomal transgene expression in liver following delivery of a scaffold/matrix attachment region containing non-viral vector. Gene Ther 2008; 15: 1593-1605.

63. Baker A, Cotten M. Delivery of bacterial artificial chromosomes into mammalian cells with psoralen-inactivated adenovirus carrier. Nucleic Acids Res 1997; 25: 1950-1956.

64. Westphal EM et al. A system for shuttling 200-kb BAC/PAC clones into human cells: stable extrachromosomal persistence and long-term ectopic gene activation. Hum Gene Ther 1998; 9: 1863-1873.

65. Hibbitt OC et al. Delivery and long-term expression of a $135 \mathrm{~kb}$ LDLR genomic DNA locus in vivo by hydrodynamic tail vein injection. J Gene Med 2007; 9: 488-497.

66. Stenler S et al. Gene transfer to mouse heart and skeletal muscles using a minicircle expressing human vascular endothelial growth factor. J Cardiovasc Pharmacol 2009; 53: 18-23.

67. Wu J et al. Minicircle-IFNgamma induces antiproliferative and antitumoral effects in human nasopharyngeal carcinoma. Clin Cancer Res 2006; 12: 47024713.

68. Greco $\mathrm{O}$ et al. Novel chimeric gene promoters responsive to hypoxia and ionizing radiation. Gene Ther 2002; 9: 1403-1411.

69. Bauerschmitz GJ et al. Tissue-specific promoters active in CD44+CD24-/low breast cancer cells. Cancer Res 2008; 68: 5533-5539.

70. Pujal J et al. Keratin 7 promoter selectively targets transgene expression to normal and neoplastic pancreatic ductal cells in vitro and in vivo. Faseb J 2009; 23: 1366-1375.

71. Pastore L et al. Use of a liver-specific promoter reduces immune response to the transgene in adenoviral vectors. Hum Gene Ther 1999; 10: 1773-1781.

72. Li X, Eastman EM, Schwartz RJ, Draghia-Akli R. Synthetic muscle promoters: activities exceeding naturally occurring regulatory sequences. Nat Biotechnol 1999; 17: 241-245.

73. Wang Y et al. Positive and negative regulation of gene expression in eukaryotic cells with an inducible transcriptional regulator. Gene Ther 1997; 4: 432-441.

74. Gossen $\mathrm{M}$ et al. Transcriptional activation by tetracyclines in mammalian cells. Science 1995; 268: 1766-1769.

75. Verma IM. Gene therapy. Sci Am 1990; 263: 68-72, 81-4.

76. Barzelay A et al. A potential role for islet-1 in post-natal angiogenesis and vasculogenesis. Thromb Haemost 2010; 103: 188-197. 
77. Lewis P, Hensel M, Emerman M. Human immunodeficiency virus infection of cells arrested in the cell cycle. Embo $J$ 1992; 11: 3053-3058.

78. Bergelson JM et al. Isolation of a common receptor for Coxsackie B viruses and adenoviruses 2 and 5. Science 1997; 275: 1320-1323.

79. Miyoshi H, Takahashi M, Gage FH, Verma IM. Stable and efficient gene transfer into the retina using an HIV-based lentiviral vector. Proc Natl Acad Sci U S A 1997; 94: 10319-10323.

80. Hofmann A et al. Combined targeting of lentiviral vectors and positioning of transduced cells by magnetic nanoparticles. Proc Natl Acad Sci U S A 2009; 106: 44-49.

81. Apolonia L et al. Stable gene transfer to muscle using non-integrating lentiviral vectors. Mol Ther 2007; 15: 1947-1954.

82. Kojaoghlanian $\mathrm{T}$ et al. Lentivectors encoding immunosuppressive proteins genetically engineer pancreatic beta-cells to correct diabetes in allogeneic mice. Gene Ther 2009; 16: 340-348.

83. Guo YH et al. Hepatocyte growth factor and granulocyte colony-stimulating factor form a combined neovasculogenic therapy for ischemic cardiomyopathy. Cytotherapy 2008; 10: 857-867.

84. Yeh P, Perricaudet M. Advances in adenoviral vectors: from genetic engineering to their biology. Faseb $J 1997 ; 11: 615-623$.

85. Dai Y et al. Cellular and humoral immune responses to adenoviral vectors containing factor IX gene: tolerization of factor IX and vector antigens allows for long-term expression. Proc Natl Acad Sci U S A 1995; 92: 1401-1405.

86. Yang Y, Ertl HC, Wilson JM. MHC class I-restricted cytotoxic T lymphocytes to viral antigens destroy hepatocytes in mice infected with E1-deleted recombinant adenoviruses. Immunity 1994; 1: 433-442.

87. Engelhardt JF, Ye X, Doranz B, Wilson JM. Ablation of E2A in recombinant adenoviruses improves transgene persistence and decreases inflammatory response in mouse liver. Proc Natl Acad Sci U S A 1994; 91: 6196-6200.

88. Chen $\mathrm{HH}$ et al. Persistence in muscle of an adenoviral vector that lacks all viral genes. Proc Natl Acad Sci U S A 1997; 94: 1645-1650.

89. Wickham TJ. Targeting adenovirus. Gene Ther 2000; 7: 110-114.

90. Mizuguchi H, Hayakawa T. Targeted adenovirus vectors. Hum Gene Ther 2004; 15: 1034-1044.

91. Kurachi S et al. Characterization of capsid-modified adenovirus vectors containing heterologous peptides in the fiber knob, protein IX, or hexon. Gene Ther 2007; 14: 266-274.

92. Tomko RP, Xu R, Philipson L. HCAR and MCAR: the human and mouse cellular receptors for subgroup $\mathrm{C}$ adenoviruses and group B coxsackieviruses. Proc Natl Acad Sci U S A 1997; 94: 3352-3356.

93. Staba MJ, Wickham TJ, Kovesdi I, Hallahan DE. Modifications of the fiber in adenovirus vectors increase tropism for malignant glioma models. Cancer Gene Ther 2000; 7: 13-19.

94. Dmitriev I et al. An adenovirus vector with genetically modified fibers demonstrates expanded tropism via utilization of a coxsackievirus and adenovirus receptor-independent cell entry mechanism. J Virol 1998; 72: 9706-9713. 
95. Koizumi $\mathrm{N}$ et al. Generation of fiber-modified adenovirus vectors containing heterologous peptides in both the HI loop and C terminus of the fiber knob. $J$ Gene Med 2003; 5: 267-276.

96. Dmitriev IP, Kashentseva EA, Curiel DT. Engineering of adenovirus vectors containing heterologous peptide sequences in the $\mathrm{C}$ terminus of capsid protein IX. J Virol 2002; 76: 6893-6899.

97. Vigne E et al. RGD inclusion in the hexon monomer provides adenovirus type 5based vectors with a fiber knob-independent pathway for infection. $J$ Virol 1999; 73: 5156-5161.

98. Biermann V et al. Targeting of high-capacity adenoviral vectors. Hum Gene Ther 2001; 12: 1757-1769.

99. Chorny $\mathrm{M}$ et al. Adenoviral gene vector tethering to nanoparticle surfaces results in receptor-independent cell entry and increased transgene expression. Mol Ther 2006; 14: 382-391.

100. Cheng K et al. Adenovirus-based vascular endothelial growth factor gene delivery to human pancreatic islets. Gene Ther 2004; 11: 1105-1116.

101. Panakanti R, Mahato RI. Bipartite Vector Encoding hVEGF and hIL-1Ra for ex Vivo Transduction into Human Islets. Mol Pharm 2008.

102. Christofori G, Naik P, Hanahan D. Vascular endothelial growth factor and its receptors, flt-1 and flk-1, are expressed in normal pancreatic islets and throughout islet cell tumorigenesis. Mol Endocrinol 1995; 9: 1760-1770.

103. Gannon $\mathrm{G}$ et al. Overexpression of vascular endothelial growth factor-A165 enhances tumor angiogenesis but not metastasis during beta-cell carcinogenesis. Cancer Res 2002; 62: 603-608.

104. Beattie GM et al. A novel approach to increase human islet cell mass while preserving beta-cell function. Diabetes 2002; 51: 3435-3439.

105. Muzyczka N. Use of adeno-associated virus as a general transduction vector for mammalian cells. Curr Top Microbiol Immunol 1992; 158: 97-129.

106. Verma IM, Somia N. Gene therapy -- promises, problems and prospects. Nature 1997; 389: 239-242.

107. Xu R et al. Diabetes gene therapy: potential and challenges. Curr Gene Ther 2003; 3: 65-82.

108. Craig AT et al. Transduction of rat pancreatic islets with pseudotyped adenoassociated virus vectors. Virol J 2009; 6: 61.

109. Carter JD et al. Viral IL-10-mediated immune regulation in pancreatic islet transplantation. Mol Ther 2005; 12: 360-368.

110. Dukkipati A et al. BacMam system for high-level expression of recombinant soluble and membrane glycoproteins for structural studies. Protein Expr Purif 2008; 62: 160-170.

111. Schlesinger S, Dubensky TW. Alphavirus vectors for gene expression and vaccines. Curr Opin Biotechnol 1999; 10: 434-439.

112. Schlesinger S. Alphavirus vectors: development and potential therapeutic applications. Expert Opin Biol Ther 2001; 1: 177-191.

113. Felgner PL et al. Lipofection: a highly efficient, lipid-mediated DNA-transfection procedure. Proc Natl Acad Sci U S A 1987; 84: 7413-7417. 
114. Gao H, Hui KM. Synthesis of a novel series of cationic lipids that can act as efficient gene delivery vehicles through systematic heterocyclic substitution of cholesterol derivatives. Gene Ther 2001; 8: 855-863.

115. Lee ER et al. Detailed analysis of structures and formulations of cationic lipids for efficient gene transfer to the lung. Hum Gene Ther 1996; 7: 1701-1717.

116. van der Woude I et al. Novel pyridinium surfactants for efficient, nontoxic in vitro gene delivery. Proc Natl Acad Sci US A 1997; 94: 1160-1165.

117. Hong $\mathrm{M}$ et al. Efficient tumor targeting of hydroxycamptothecin loaded PEGylated niosomes modified with transferrin. J Control Release 2009; 133: 96102.

118. Lee RJ, Huang L. Folate-targeted, anionic liposome-entrapped polylysinecondensed DNA for tumor cell-specific gene transfer. J Biol Chem 1996; 271: 8481-8487.

119. Remy JS et al. Targeted gene transfer into hepatoma cells with lipopolyaminecondensed DNA particles presenting galactose ligands: a stage toward artificial viruses. Proc Natl Acad Sci U S A 1995; 92: 1744-1748.

120. Chae HY et al. Effective glycemic control achieved by transplanting non-viral cationic liposome-mediated VEGF-transfected islets in streptozotocin-induced diabetic mice. Exp Mol Med 2005; 37: 513-523.

121. Yang H, McAlister VC, Al-Jazaeri A, Wright JR, Jr. Liposomal encapsulation significantly enchances the immunosuppressive effect of tacrolimus in a discordant islet xenotransplant model. Transplantation 2002; 73: 710-713.

122. Wadhwa MS et al. Peptide-mediated gene delivery: influence of peptide structure on gene expression. Bioconjug Chem 1997; 8: 81-88.

123. Zauner $\mathrm{W}$ et al. Glycerol and polylysine synergize in their ability to rupture vesicular membranes: a mechanism for increased transferrin-polylysine-mediated gene transfer. Exp Cell Res 1997; 232: 137-145.

124. Haider M, Megeed Z, Ghandehari H. Genetically engineered polymers: status and prospects for controlled release. J Control Release 2004; 95: 1-26.

125. Wagner E. Application of membrane-active peptides for nonviral gene delivery. Adv Drug Deliv Rev 1999; 38: 279-289.

126. Hara $\mathrm{T}$ et al. Effects of fusogenic and DNA-binding amphiphilic compounds on the receptor-mediated gene transfer into hepatic cells by asialofetuin-labeled liposomes. Biochim Biophys Acta 1996; 1278: 51-58.

127. Esbjorner EK et al. Membrane binding of $\mathrm{pH}$-sensitive influenza fusion peptides. positioning, configuration, and induced leakage in a lipid vesicle model.

Biochemistry 2007; 46: 13490-13504.

128. Kichler A, Mechtler K, Behr JP, Wagner E. Influence of membrane-active peptides on lipospermine/DNA complex mediated gene transfer. Bioconjug Chem 1997; 8: 213-221.

129. Kamata H, Yagisawa H, Takahashi S, Hirata H. Amphiphilic peptides enhance the efficiency of liposome-mediated DNA transfection. Nucleic Acids Res 1994; 22: 536-537.

130. Wilke $\mathrm{M}$ et al. Efficacy of a peptide-based gene delivery system depends on mitotic activity. Gene Ther 1996; 3: 1133-1142. 
131. Teramura Y, Iwata H. Islets surface modification prevents blood-mediated inflammatory responses. Bioconjug Chem 2008; 19: 1389-1395.

132. Boussif $\mathrm{O}$ et al. A versatile vector for gene and oligonucleotide transfer into cells in culture and in vivo: polyethylenimine. Proc Natl Acad Sci U S A 1995; 92: 7297-7301.

133. Wang DA et al. Novel branched poly(ethylenimine)-cholesterol water-soluble lipopolymers for gene delivery. Biomacromolecules 2002; 3: 1197-1207.

134. Mahato RI et al. Cationic lipid and polymer-based gene delivery to human pancreatic islets. Mol Ther 2003; 7: 89-100.

135. Carlisle RC et al. Adenovirus hexon protein enhances nuclear delivery and increases transgene expression of polyethylenimine/plasmid DNA vectors. Mol Ther 2001; 4: 473-483.

136. Croyle MA et al. PEGylated helper-dependent adenoviral vectors: highly efficient vectors with an enhanced safety profile. Gene Ther 2005; 12: 579-587.

137. Yotnda $\mathrm{P}$ et al. Bilamellar cationic liposomes protect adenovectors from preexisting humoral immune responses. Mol Ther 2002; 5: 233-241.

138. Price AR, Limberis MP, Wilson JM, Diamond SL. Pulmonary delivery of adenovirus vector formulated with dexamethasone-spermine facilitates homologous vector re-administration. Gene Ther 2007; 14: 1594-1604.

139. Subr V et al. Coating of adenovirus type 5 with polymers containing quaternary amines prevents binding to blood components. J Control Release 2009; 135: $152-$ 158.

140. Wu GY, Wu CH. Evidence for targeted gene delivery to Hep G2 hepatoma cells in vitro. Biochemistry 1988; 27: 887-892.

141. Wu GY, Wu CH. Receptor-mediated gene delivery and expression in vivo. J Biol Chem 1988; 263: 14621-14624.

142. Zatloukal K et al. Transferrinfection: a highly efficient way to express gene constructs in eukaryotic cells. Ann N Y Acad Sci 1992; 660: 136-153.

143. Berney T, Ricordi C. Islet cell transplantation: the future? Langenbecks Arch Surg 2000; 385: 373-378.

144. Menger MD, Vajkoczy P, Beger C, Messmer K. Orientation of microvascular blood flow in pancreatic islet isografts. J Clin Invest 1994; 93: 2280-2285.

145. Vajkoczy P, Menger MD, Simpson E, Messmer K. Angiogenesis and vascularization of murine pancreatic islet isografts. Transplantation 1995; 60: 123-127.

146. Lopez-Talavera JC et al. Hepatocyte growth factor gene therapy for pancreatic islets in diabetes: reducing the minimal islet transplant mass required in a glucocorticoid-free rat model of allogeneic portal vein islet transplantation. Endocrinology 2004; 145: 467-474.

147. Cardozo AK et al. IL-1beta and IFN-gamma induce the expression of diverse chemokines and IL-15 in human and rat pancreatic islet cells, and in islets from pre-diabetic NOD mice. Diabetologia 2003; 46: 255-266.

148. Lee DY, Park SJ, Nam JH, Byun Y. A combination therapy of PEGylation and immunosuppressive agent for successful islet transplantation. J Control Release 2006; 110: 290-295. 
149. Tellez $\mathrm{N}$ et al. Adenoviral overexpression of interleukin-1 receptor antagonist protein increases beta-cell replication in rat pancreatic islets. Gene Ther 2005; 12: 120-128.

150. Giannoukakis $\mathrm{N}$ et al. Adenoviral gene transfer of the interleukin-1 receptor antagonist protein to human islets prevents IL-1beta-induced beta-cell impairment and activation of islet cell apoptosis in vitro. Diabetes 1999; 48: 1730-1736.

151. Sandberg JO, Eizirik DL, Sandler S. IL-1 receptor antagonist inhibits recurrence of disease after syngeneic pancreatic islet transplantation to spontaneously diabetic non-obese diabetic (NOD) mice. Clin Exp Immunol 1997; 108: 314-317.

152. Welsh N, Bendtzen K, Welsh M. Expression of an insulin/interleukin-1 receptor antagonist hybrid gene in insulin-producing cell lines (HIT-T15 and NIT-1) confers resistance against interleukin-1-induced nitric oxide production. J Clin Invest 1995; 95: 1717-1722.

153. Bertera $\mathrm{S}$ et al. Gene combination transfer to block autoimmune damage in transplanted islets of Langerhans. Exp Diabesity Res 2004; 5: 201-210.

154. Mistry SJ, Bank A, Atweh GF. Targeting stathmin in prostate cancer. Mol Cancer Ther 2005; 4: 1821-1829.

155. Silvertown JD, Geddes BJ, Summerlee AJ. Adenovirus-mediated expression of human prorelaxin promotes the invasive potential of canine mammary cancer cells. Endocrinology 2003; 144: 3683-3691.

156. Ajioka I, Akaike T, Watanabe Y. Expression of vascular endothelial growth factor promotes colonization, vascularization, and growth of transplanted hepatic tissues in the mouse. Hepatology 1999; 29: 396-402.

157. Jansen RC, Nap JP, Mlynarova L. Errors in genomics and proteomics. Nat Biotechnol 2002; 20: 19.

158. Crystal RG et al. Analysis of risk factors for local delivery of low- and intermediate-dose adenovirus gene transfer vectors to individuals with a spectrum of comorbid conditions. Hum Gene Ther 2002; 13: 65-100.

159. Liu D et al. Cytokines induce apoptosis in beta-cells isolated from mice lacking the inducible isoform of nitric oxide synthase (iNOS-/-). Diabetes 2000; 49: 1116-1122.

160. Goss JA et al. Achievement of insulin independence in three consecutive type-1 diabetic patients via pancreatic islet transplantation using islets isolated at a remote islet isolation center. Transplantation 2002; 74: 1761-1766.

161. Shapiro AM et al. Islet transplantation in seven patients with type I diabetes mellitus using a glucocorticoid-free immunosuppressive regimen. $N$ Engl J Med 2000; 343: 230-238.

162. Ryan EA et al. Clinical outcomes and insulin secretion after islet transplantation with the Edmonton protocol. Diabetes 2001; 50: 710-719.

163. Vajkoczy P et al. Histogenesis and ultrastructure of pancreatic islet graft microvasculature. Evidence for graft revascularization by endothelial cells of host origin. Am J Pathol 1995; 146: 1397-1405.

164. Garcia-Ocana A et al. Transgenic overexpression of hepatocyte growth factor in the beta-cell markedly improves islet function and islet transplant outcomes in mice. Diabetes 2001; 50: 2752-2762. 
165. Garcia-Ocana A et al. Adenovirus-mediated hepatocyte growth factor expression in mouse islets improves pancreatic islet transplant performance and reduces beta cell death. J Biol Chem 2003; 278: 343-351.

166. Benns JM et al. $\mathrm{pH}$-sensitive cationic polymer gene delivery vehicle: N-Acpoly(L-histidine)-graft-poly(L-lysine) comb shaped polymer. Bioconjug Chem 2000; 11: 637-645.

167. Roccisana J et al. Targeted inactivation of hepatocyte growth factor receptor cmet in beta-cells leads to defective insulin secretion and GLUT-2 downregulation without alteration of beta-cell mass. Diabetes 2005; 54: 2090-2102.

168. Fontaine MJ, Fan W. Islet cell transplantation as a cure for insulin dependent diabetes: current improvements in preserving islet cell mass and function.

Hepatobiliary Pancreat Dis Int 2003; 2: 170-179.

169. Boros P, Miller CM. Hepatocyte growth factor: a multifunctional cytokine. Lancet 1995; 345: 293-295.

170. Danthinne X, Imperiale MJ. Production of first generation adenovirus vectors: a review. Gene Ther 2000; 7: 1707-1714.

171. Wang Q, Finer MH. Second-generation adenovirus vectors. Nat Med 1996; 2: 714-716.

172. Yang Y et al. Cellular immunity to viral antigens limits E1-deleted adenoviruses for gene therapy. Proc Natl Acad Sci U S A 1994; 91: 4407-4411.

173. Zsengeller ZK et al. Persistence of replication-deficient adenovirus-mediated gene transfer in lungs of immune-deficient (nu/nu) mice. Hum Gene Ther 1995; 6: $457-467$.

174. Nakano $\mathrm{M}$ et al. Hepatocyte growth factor is essential for amelioration of yperglycemia in streptozotocin-induced diabetic mice receiving a marginal mass of intrahepatic islet grafts. Transplantation 2000; 69: 214-221.

175. Ramachandran S et al. Improved islet yields from pancreas preserved in perflurocarbon is via inhibition of apoptosis mediated by mitochondrial pathway. Am J Transplant 2006; 6: 1696-1703.

176. Hanke J. Apoptosis and occurrence of Bcl-2, Bak, Bax, Fas and FasL in the developing and adult rat endocrine pancreas. Anat Embryol (Berl) 2000; 202: 303-312.

177. Jiang X, Wang X. Cytochrome C-mediated apoptosis. Annu Rev Biochem 2004; 73: 87-106.

178. Bataller R, Brenner DA. Liver fibrosis. J Clin Invest 2005; 115: 209-218.

179. Gines P, Cardenas A, Arroyo V, Rodes J. Management of cirrhosis and ascites. $N$ Engl J Med 2004; 350: 1646-1654.

180. Inagaki $\mathrm{Y}$ et al. Regulation of the [alpha]2(I) collagen gene transcription in fatstoring cells derived from a cirrhotic liver. Hepatology 1995; 22: 573-579.

181. Parsons CJ, Takashima M, Rippe RA. Molecular mechanisms of hepatic fibrogenesis. J Gastroenterol Hepatol 2007; 22: S79-S84.

182. Arias $\mathrm{M}$ et al. Adenoviral delivery of an antisense RNA complementary to the $3^{\prime}$ coding sequence of transforming growth factor- $\{$ beta $\} 1$ inhibits fibrogenic activities of hepatic stellate cells. Cell Growth Differ 2002; 13: 265-273. 
183. Liu C et al. Smads 2 and 3 are differentially activated by transforming growth factor- $\beta$ (TGF- $\beta$ ) in quiescent and activated hepatic stellate cells. $J$ Biol Chem 2003; 278: 11721-11728.

184. Marra F et al. Extracellular signal-regulated kinase activation differentially regulates platelet-derived growth factor's actions in hepatic stellate cells, and is induced by in vivo liver injury in the rat. Hepatology 1999; 30: 951-958.

185. Nakatsukasa $\mathrm{H}$ et al. Cellular distribution of transforming growth factor-beta 1 and procollagen types I, III, and IV transcripts in carbon tetrachloride-induced rat liver fibrosis. The J Clin Invest 1990; 85: 1833-1843.

186. Ramos C et al. Fgf-1 reverts epithelial-mesenchymal transition induced by tgf\{beta\} 1 through mapk/erk kinase pathway. Am J Physiol Lung Cell Mol Physiol. 2010; 299: L222-L231.

187. Soriano V et al. Regression of liver fibrosis in hepatitis $\mathrm{C}$ virus/HIV-co-infected patients after treatment with pegylated interferon plus ribavirin. AIDS 2006; 20: 2175-2181.

188. Arthur MJP. Reversibility of liver fibrosis and cirrhosis following treatment for hepatitis C. Gastroenterology 2002; 122: 1525-1528.

189. Knauert MP, Glazer PM. Triplex forming oligonucleotides: sequence-specific tools for gene targeting. Hum. Mol. Genet. 2001; 10: 2243-2251.

190. Svinarchuk F et al. Recruitment of transcription factors to the target site by triplex- forming oligonucleotides. Nucl. Acids Res. 1997; 25: 3459-3464.

191. Ye Z, Guntaka RV, Mahato RI. Sequence-specific triple helix formation with genomic DNA. Biochemistry 2007; 46: 11240-11252.

192. Xia J-L, Dai C, Michalopoulos GK, Liu Y. Hepatocyte growth factor attenuates liver fibrosis induced by bile duct ligation. Am J Pathol 2006; 168: 1500-1512.

193. Uchinami H, Seki E, Brenner DA, D'Armiento J. Loss of MMP 13 attenuates murine hepatic injury and fibrosis during cholestasis. Hepatology 2006; 44: 420429.

194. Leonie B, Klaas P, Grietje M, Dirk KFM. Targeting of sugar- and chargemodified albumins to fibrotic rat livers: the accessibility of hepatic cells after chronic bile duct ligation. J hepatol 1998; 29: 579-588.

195. Ramon B et al. NADPH oxidase signal transduces angiotensin II in hepatic stellate cells and is critical in hepatic fibrosis. J Clin Invest 2003; 112: 1383-1394.

196. Bissell DM, Roulot D, George J. Transforming growth factor beta and the liver. Hepatology 2001; 34: 859-867.

197. Paradis V et al. Expression of connective tissue growth factor in experimental rat and human liver fibrosis. Hepatology 1999; 30: 968-976.

198. Roderfeld $\mathrm{M}$ et al. Inhibition of hepatic fibrogenesis by matrix metalloproteinase9 mutants in mice. FASEB J. 2006; 20: 444-454.

199. Kivirikko KI, Laitinen O, Prockop DJ. Modifications of a specific assay for hydroxyproline in urine. Anal Biochem 1967; 19: 249-255.

200. Richard AR, David AB. From quiescence to activation: gene regulation in hepatic stellate cells. Gastroenterology 2004; 127: 1260-1262.

201. Cheng K, Mahato RI. Gene modulation for treating liver fibrosis. Crit Rev Ther Drug Carrier Syst 2007; 24: 93-146. 
202. Honda E, Yoshida K, Munakata H. Transforming growth factor-beta upregulates the expression of integrin and related proteins in MRC-5 human myofibroblasts. Tohoku J Exp Med; 220: 319-327.

203. Cheng K, Ye Z, Guntaka RV, Mahato RI. Biodistribution and hepatic uptake of triplex-forming oligonucleotides against type alpha1(I) collagen gene promoter in normal and fibrotic rats. Mol Pharm 2005; 2: 206-217.

204. Gabele E et al. TNFalpha is required for cholestasis-induced liver fibrosis in the mouse. Biochem Biophys Res Commun 2009; 378: 348-353.

205. Xia JL, Dai C, Michalopoulos GK, Liu Y. Hepatocyte growth factor attenuates liver fibrosis induced by bile duct ligation. Am J Pathol 2006; 168: 1500-1512.

206. Kojima $\mathrm{T}$ et al. Transforming growth factor-beta induces epithelial to mesenchymal transition by down-regulation of claudin-1 expression and the fence function in adult rat hepatocytes. Liver Int 2008; 28: 534-545.

207. Caja L et al. Differential intracellular signalling induced by TGF-beta in rat adult hepatocytes and hepatoma cells: implications in liver carcinogenesis. Cell Signal 2007; 19: 683-694.

208. Natsuizaka $\mathrm{M}$ et al. Insulin-like growth factor binding protein-3 promotes transforming growth factor-\{beta $\} 1$-mediated epithelial-to-mesenchymal transition and motility in transformed human esophageal cells. Carcinogenesis 2010; 8: 1344-1353. 


\section{VITA}

Ravikiran Panakanti was born in Adilabad, Andhra Pradesh, India, in 1982. He joined the Shadan College of Pharmacy in 2000 and received a Bachelor of Pharmacy degree in 2004. In the Fall of 2005, he joined the graduate program in the Department of Pharmaceutical Sciences, the University of Tennessee Health Science Center, Memphis, from where he will receive his Ph.D. (2010) under the supervision of Prof. Ram I. Mahato.

\section{RESEARCH PUBLICATIONS}

- Panakanti R and Mahato RI. Bipartite vector encoding hVEGF and hIL-1Ra for ex vivo transduction into human islets. Mol Pharm 2009; 6: 274-284.

- Panakanti R and Mahato RI. Bipartite adenoviral vector encoding hHGF and hIL-1Ra for improved human islet transplantation. Pharm Res 2009; 26: 587596.

- Panakanti R, Yang N, Pratap A and Mahato RI. Triplex forming oligonucleotides against type $\alpha 1$ (I) collagen attenuates liver fibrosis induced by bile duct ligation. Biochem Pharmacol 2010; 80: 1718-1726.

- Pratap A, Panakanti R, Yang N, Eason JD, Mahato RI. Inhibition of endogenous hedgehog signaling protects against acute liver injury after ischemia reperfusion. Pharm Res 2010; 27: 2492-2504.

- Wu H, Panakanti R, Li F, and Mahato RI. XIAP gene expression protects $\beta$-cells and human islets from apoptotic cell death. Mol Pharm.2010; epub.

\section{BOOK CHAPTER}

- Panakanti R and Mahato RI (2009) Recent advances in gene expression and delivery systems. In: Narang AS and Mahato RI (eds) Targeted Delivery of Small and Macromolecular Drugs, CRC Press, Inc. Boca Raton, FL.

\section{RESEARCH PRESENTATIONS}

- Li F, Panakanti R and Mahato RI (2007) Gene expression and silencing for successful islet transplantation. American Society of Gene Therapy, Seattle, WA.

- Mahato RI, Panakanti R, Li F and Cheng G (2009) Gene delivery and silencing for improved islet transplantation. 14th International Symposium on Recent Advances in Drug Delivery Systems, Salt Lake City, UT.

- Panakanti R, Cheng G and Mahato RI (2009) Construction of bicistronic adenoviral vector encoding genes hHGF and hIL-1Ra for islet transplantation. AAPS Annual Meeting, Atlanta, GA.

- Panakanti R, Cheng G and Mahato RI (2008) Bicistronic adenoviral vector encoding hVEGF and hIL-1Ra for improving islet transplantation. American Society of Gene Therapy, Boston, MA. 
- Panakanti R and Mahato RI (2009) E1, E3 and E4 deleted bipartite adenoviral vector encoding hVEGF and hIL-1Ra for decreased immunogenicity and improved islet function. AAPS Annual Meeting (Los Angeles, CA). Received AAPS travel award.

\section{AWARDS AND HONORS}

- AAPS Biotech student travel award-2009.

- University of Tennessee student travel award-2009.

- Imhotep Society, UTHSC. 